\title{
Synthesis of Aspidodispermine via Pericyclic Framework Reconstruction
}

\author{
F. Reuß and P. Heretsch* \\ Institute of Chemistry and Biochemistry, Freie Universität Berlin, \\ Takustraße 3, 14195 Berlin, Germany \\ ${ }^{*}$ Correspondence to: philipp.heretsch@fu-berlin.de
}

Supporting Information

\section{Table of Contents}

1 General Methods $\quad$ S2

2 Experimental Procedures and Characterization Data $\quad$ S4

2.1 Synthesis of Pyrroloquinoline Scaffold $12 \ldots \ldots \ldots \ldots$. . . . . . . . . . S4

2.2 Synthesis of Hydrazine $13 \ldots \ldots \ldots \ldots$. . . . . . . . . . . . . . . . . . . . . .

2.3 Fragment Coupling . . . . . . . . . . . . . . . . . . . . . . . . S22

3 References $\quad$ S27

4 NMR Spectra $\quad$ S28

4.1 New Compounds . . . . . . . . . . . . . . . . . . . . . . . . . . s28

4.2 Known Compounds . . . . . . . . . . . . . . . . . . . s51 


\section{General Methods}

All reactions sensitive to moisture and/or air were carried out using heat-gun dried glassware, an argon atmosphere, and dry solvents. Dry $\mathrm{CH}_{2} \mathrm{Cl}_{2}, \mathrm{Et}_{2} \mathrm{O}$ and $\mathrm{PhMe}$ were taken from a $\mathrm{M}$. Braun GmbH MB SPS-800 solvent purification system. THF was distilled from sodium and stored over $4 \AA$ molecular sieves. Dry $\mathrm{MeOH}$ was purchased from Acros (extra dry quality). $\mathrm{Me}_{2} \mathrm{CO}$, EtOAc and nhexane were purified by distillation on a rotary evaporator. All other solvents and commercially available reagents were used without further purification unless otherwise stated.

Reactions were monitored by thin-layer chromatography (TLC) carried out on Merck Silica Gel $50 \mathrm{~F}_{245}$-plates and visualized by fluorescence quenching under UV-light or an aqueous solution of cerium sulfate and phosphomolybdic acid (CAM) or an alkaline potassium permanganate solution and heat as developing agent. Column chromatographic purification was always performed on Macherey-Nagel Silica Gel 60 M (40 - $60 \mu \mathrm{m})$.

Concentration under reduced pressure was performed by rotary evaporation at $45^{\circ} \mathrm{C}$ and appropriate pressure and by exposure to high vacuum $\left(1 \times 10^{-3} \mathrm{mbar}\right)$ at room temperature.

NMR spectra were recorded on either a Jeol ECX400 (400 MHz), a Jeol ECP500 (500 MHz), a Bruker AVANCE III 500 (500 MHz), a Jeol ECZ600 (600 MHz) or a Bruker AVANCE III 700 (700 MHz, with CryoProbe) spectrometer. Chemical shifts $\delta$ are reported in parts per million (ppm) and are calibrated using residual undeuterated solvent $\left(\mathrm{CDCl}_{3}:{ }^{1} \mathrm{H} \delta=7.26 \mathrm{ppm}\right.$, ${ }^{13} \mathrm{C} \delta=77.16 \mathrm{ppm} ; \mathrm{CD}_{3} \mathrm{CN}:{ }^{1} \mathrm{H} \delta=1.94 \mathrm{ppm},{ }^{13} \mathrm{C} \delta=1.32 \mathrm{ppm} ;\left(\mathrm{D}_{3} \mathrm{C}\right)_{2} \mathrm{SO}:{ }^{1} \mathrm{H} \delta=2.50 \mathrm{ppm}$, ${ }^{13} \mathrm{C} \delta=39.51 \mathrm{ppm}$ ) as an internal reference at $298 \mathrm{~K}$ unless otherwise noted. The given multiplicities are phenomenological, thus the actual appearance of the signals is stated and not the theoretically expected one. The following abbreviations were used to designate multiplicities: $\mathrm{s}=$ singlet, $\mathrm{bs}=$ broad singlet, $\mathrm{d}=$ doublet, $\mathrm{t}=$ triplet, $\mathrm{q}=$ quartet, $\mathrm{p}=$ pentet. In case no multiplicity could be identified, the chemical shift range of the signal is given ( $m=$ multiplet). Infrared (IR) spectra were measured on a Jasco FT/IR-4100 Type A spectrometer with a TGS detector. Wavenumbers $\tilde{\nu}$ are given in $\mathrm{cm}^{-1}$.

High-resolution mass spectra (HRMS) were recorded using an Agilent 6210 ESI-TOF or an Ionspec QFT-7 ESI-TOF spectrometer.

Melting points were measured on a Reichert Thermovar Kofler hot-stage-microscope.

Irradiation with UV-light was performed with a water-cooled Hg-medium pressure lamp TQ-718 from Heraeus with electronic control gear P-EVG 10 from Peschl Ultraviolett. Reactions were 
perfomed by placing a non-cooled reaction vessel next to the lamp (distance $2 \mathrm{~cm}$ ).

Flow experiments were performed with the following equipment: Tubings made from FEP (outer diameter 1/16", inner diameter 1/32"), PTFE (outer diameter 1/8", inner diameter 1/16") and PEEK (outer diameter 1/16", inner diameter $250 \mu \mathrm{m}$ ) were obtained from BOLA. Tubings and mixers were connected using either coned 10-32 UNF fittings made from stainless steel 316 L from UPCHURCH SCIENTIFIC or flat bottom $1 / 428$ UNF gripper fittings made from PP from DIBAFIT. Adapters for $1 / 428$ UNF systems were made from PP or ETFE and were provided from UPCHURCH SCIENTIFIC. As liquid delivery, a WATERS 515 HPLC pump was used. 


\section{Experimental Procedures and Characterization Data}

\subsection{Synthesis of Pyrroloquinoline Scaffold 12}

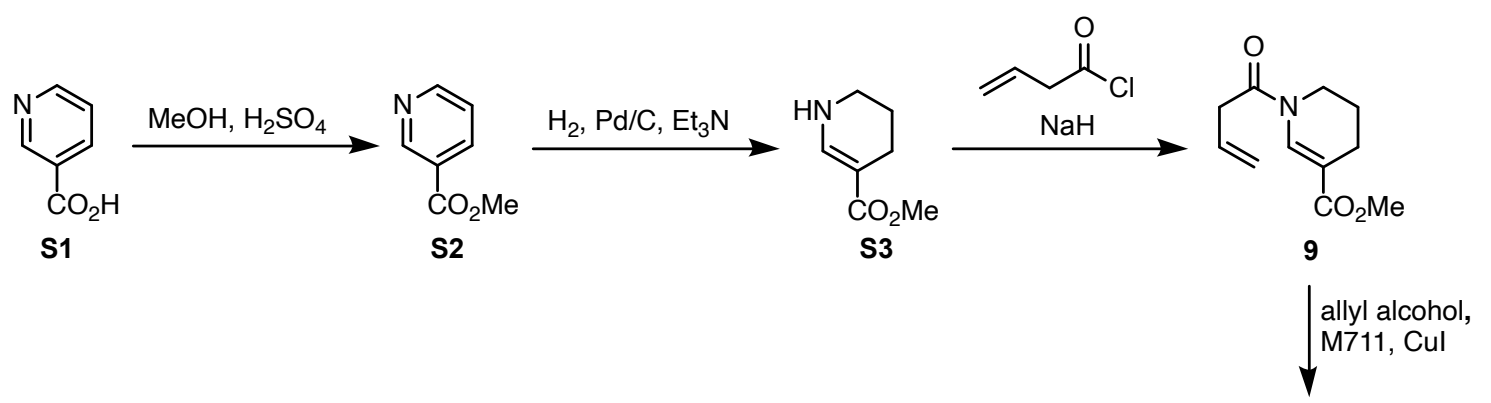<smiles>COCC1C2CC(=O)N3CCCC1(COC)C23</smiles>

S5

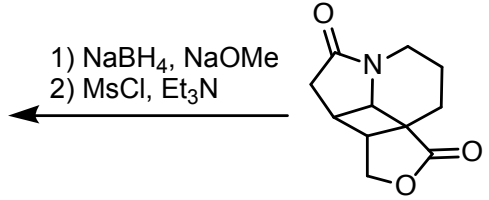

10
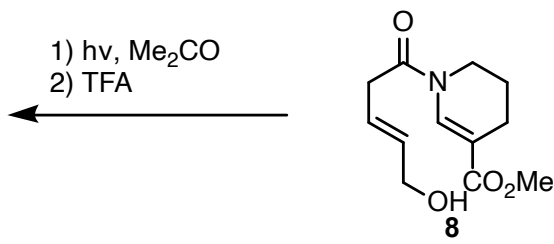<smiles></smiles><smiles>CCCN1C(=O)CC2C3CSCC3(C)C21</smiles>

S6<smiles>O=C1CC2C3CS(=S)CC34CCCN1C24</smiles>

7 Ö
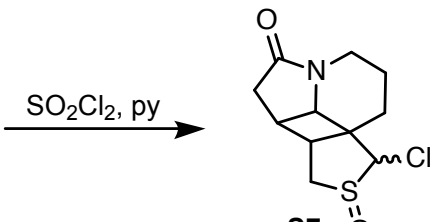

s7

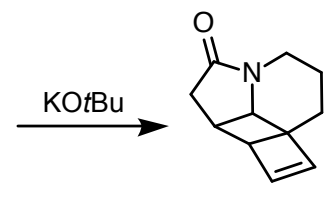

6

$110^{\circ} \mathrm{C}$

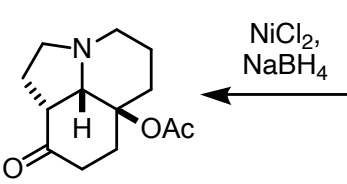

12<smiles>CC(=O)OC12C=CC(=O)[C@H]3CC(=S)N4CCCC1(C3)C42</smiles>

S8
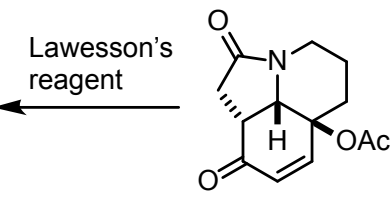

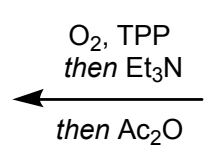

$\mathrm{Ac}_{2} \mathrm{O}$

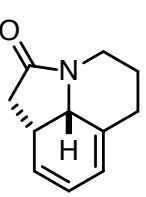

Methyl nicotinate (S2) ${ }^{[1]}$<smiles>O=C(O)c1cccnc1</smiles>

S1

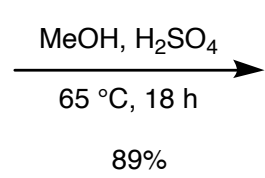<smiles>COc1cccnc1</smiles>

Conc. $\mathrm{H}_{2} \mathrm{SO}_{4}$ (70 mL, $1.3 \mathrm{~mol}, 8.0$ eq.) was added dropwise to a suspension of nicotinic acid (S1) $(20.0 \mathrm{~g}, 162 \mathrm{mmol}, 1.0 \mathrm{eq}$.) in $\mathrm{MeOH}(340 \mathrm{~mL})$ and the resulting solution was stirred at $65^{\circ} \mathrm{C}$ for $18 \mathrm{~h}$. The mixture was allowed to cool to $23^{\circ} \mathrm{C}$, diluted with $\mathrm{H}_{2} \mathrm{O}(300 \mathrm{~mL})$, and 
neutralized with solid $\mathrm{NaHCO}_{3}$. The aqueous phase was extracted with $\mathrm{CH}_{2} \mathrm{Cl}_{2}(3 \times 100 \mathrm{~mL})$ and the combined organic phases were dried over $\mathrm{MgSO}_{4}$. The solvent was removed under reduced pressure to give nicotinic acid methyl ester (S2) (19.9 g, $145 \mathrm{mmol}, 89 \%)$ as a yellow oil that solidified upon standing at $23^{\circ} \mathrm{C}$.

TLC: $\mathrm{R}_{\mathrm{F}}=0.73$ (nhexane/EtOAc 1:1, UV, CAM).

m.p.: $39-40^{\circ} \mathrm{C}\left(\mathrm{CH}_{2} \mathrm{Cl}_{2}\right)$.

${ }^{1} \mathrm{H}-\mathrm{NMR}\left(500 \mathrm{MHz}, \mathrm{CDCl}_{3}\right): \delta=9.21(\mathrm{dd}, J=2.2,1.2 \mathrm{~Hz}, 1 \mathrm{H}), 8.77-8.75(\mathrm{~m}, 1 \mathrm{H}), 8.29-8.26$ (m, 1H), 7.37 (ddq, J = 7.9, 4.8, $1.0 \mathrm{~Hz}, 1 \mathrm{H}$ ), 3.94 (s, 3H) ppm.

${ }^{13}$ C-NMR (126 MHz, $\left.\mathrm{CDCl}_{3}\right)$ : 165.6, 153.3, 150.8, 136.9, 125.9, 123.2, 52.3 ppm.

All characterization data were consistent with those reported in the literature. ${ }^{[1]}$

\section{Methyl 1,4,5,6-tetrahydropyridine-3-carboxylate (S3) ${ }^{[2]}$}

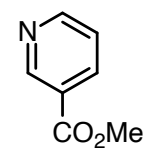

S2

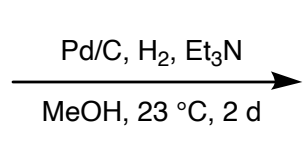

quant.

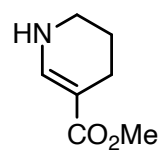

S3

To a solution of nicotinic acid methylester (S2) (10.0 g, $72.9 \mathrm{mmol}, 1.0$ eq.) in $\mathrm{MeOH}(90 \mathrm{~mL})$ were added $\mathrm{Et}_{3} \mathrm{~N}(12 \mathrm{~mL}, 88 \mathrm{mmol}, 1.2$ eq.) and $\mathrm{Pd} / \mathrm{C}(10 \% \mathrm{w} / \mathrm{w}, 1.55 \mathrm{~g}, 1.46 \mathrm{mmol}, 2 \mathrm{~mol} \%)$. After stirring at $23^{\circ} \mathrm{C}$ under an atmosphere of hydrogen (balloon) for $2 \mathrm{~d}$, the reaction mixture was filtered through a plug of Celite ${ }^{\circledR}$ and the plug was rinsed with EtOAc $(20 \mathrm{~mL})$. The solvent was removed under reduced pressure to give amine $\mathbf{S} 3$ (10.3 g, $72.9 \mathrm{mmol}$, quant.) as a colorless oil.

TLC: $\mathrm{R}_{\mathrm{F}}=0.73$ (nhexane/EtOAc 1:1, UV, CAM).

${ }^{1} \mathrm{H}-\mathrm{NMR}\left(500 \mathrm{MHz}, \mathrm{CDCl}_{3}\right): \delta=7.46(\mathrm{~d}, J=6.2 \mathrm{~Hz}, 1 \mathrm{H}), 4.35(\mathrm{~s}, 1 \mathrm{H}), 3.66(\mathrm{~s}, 3 \mathrm{H}), 3.22-3.17$ (m, 2H), 2.33 (t, $J=6.3 \mathrm{~Hz}, 2 \mathrm{H}), 1.81$ (p, $J=6.1 \mathrm{~Hz}, 2 \mathrm{H}) \mathrm{ppm}$.

${ }^{13} \mathrm{C}-\mathrm{NMR}\left(126 \mathrm{MHz}, \mathrm{CDCl}_{3}\right): \delta=169.3,143.0,95.6,50.7,40.9,21.1,20.8 \mathrm{ppm}$.

IR (ATR): $\tilde{\nu}=3337,2947,2851,1667,1613,1508,1435,1355,1316,1303,1230,1189,1176$, $1103 \mathrm{~cm}^{-1}$.

All characterization data were consistent with those reported in the literature. ${ }^{[3]}$ 


\section{Methyl 1-(but-3-enoyl)-1,4,5,6-tetrahydropyridine-3-carboxylate (9)}

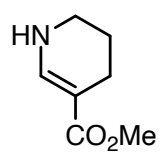

S3

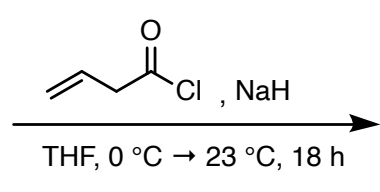

$78 \%$

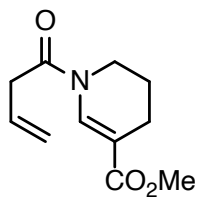

9

Oxalylchloride (13.8 mL, $160 \mathrm{mmol}, 2.2$ eq.) was added to 3-butenoic acid (12.4 mL, $146 \mathrm{mmol}$, 2.0 eq.) at $0^{\circ} \mathrm{C}$ and the resulting mixture was stirred for $3 \mathrm{~h}$ at $23^{\circ} \mathrm{C}$. In another flask, a solution of amine S3 (10.3 g, $72.9 \mathrm{mmol}, 1.0$ eq.) in dry THF $(240 \mathrm{~mL})$ was cooled to $0{ }^{\circ} \mathrm{C}, \mathrm{NaH}(60 \%$ $w / w$ in mineral oil, $6.56 \mathrm{~g}, 164 \mathrm{mmol}, 2.25 \mathrm{eq}$.) was added portionwise over $5 \mathrm{~min}$, and the reaction mixture was stirred for $30 \mathrm{~min}$ at $0^{\circ} \mathrm{C}$. The freshly prepared acid chloride was added dropwise at $0^{\circ} \mathrm{C}$, the resulting mixture was allowed to warm to $23^{\circ} \mathrm{C}$ over $18 \mathrm{~h}$, and then poured into a mixture of ice and $\mathrm{H}_{2} \mathrm{O}(200 \mathrm{~mL})$. The organic phase was separated and the aqueous phase was extracted with EtOAc $(3 \times 100 \mathrm{~mL})$. The combined organic phases were washed sequentially with $\mathrm{NaHCO}_{3}$ (sat. aq., $200 \mathrm{~mL}$ ) and brine (sat., $200 \mathrm{~mL}$ ) and dried over $\mathrm{MgSO}_{4}$. The solvent was removed under reduced pressure and the crude product was purified by column chromatography (nhexane/EtOAc 4:1). The amide 9 (11.9 g, $56.9 \mathrm{mmol}, 78 \%$ ) was obtained as a slightly yellow oil.

TLC: $\mathrm{R}_{\mathrm{F}}=0.44$ (nhexane/EtOAc 2:1, UV, CAM).

$4: 1$ mixture of rotamers, signals of the minor rotamer are marked with an *.

${ }^{1} \mathrm{H}-N M R\left(500 \mathrm{MHz}, \mathrm{CDCl}_{3}\right): \delta=8.34^{*}(\mathrm{~s}, 1 \mathrm{H}), 7.84(\mathrm{~s}, 1 \mathrm{H}), 5.95$ (ddt, $J=16.9,10.3,6.6 \mathrm{~Hz}$, $1 \mathrm{H}), 5.23-5.14(\mathrm{~m}, 2 \mathrm{H}), 3.74(\mathrm{~s}, 3 \mathrm{H}), 3.65(\mathrm{t}, J=5.9 \mathrm{~Hz}, 2 \mathrm{H}), 3.58^{*}(\mathrm{bs}, 2 \mathrm{H}), 3.35$ (d, $J=6.7 \mathrm{~Hz}, 2 \mathrm{H}), 3.26^{*}(\mathrm{bs}, 2 \mathrm{H}), 2.33(\mathrm{t}, J=6.3 \mathrm{~Hz}, 2 \mathrm{H}), 1.87^{\star}(\mathrm{bs}, 2 \mathrm{H}), 1.84-1.79(\mathrm{~m}, 2 \mathrm{H}) \mathrm{ppm}$. ${ }^{13}$ C-NMR $\left(126 \mathrm{MHz}, \mathrm{CDCl}_{3}\right): \delta=169.9,167.7,135.12,130.3,119.0,109.7,51.7,40.7,38.6$, 21.5, $20.5 \mathrm{ppm}$.

IR (ATR): $\tilde{\nu}=3337,2947,2851,1667,1613,1508,1435,1355,1316,1303,1230,1189,1176$, $1103 \mathrm{~cm}^{-1}$.

HRMS (ESI-TOF): $m / z$ calculated for $\mathrm{C}_{11} \mathrm{H}_{15} \mathrm{NO}_{3} \mathrm{Na}^{+}[\mathrm{M}+\mathrm{Na}]^{+}:$232.0944; found 232.0943 . 
Methyl (E)-1-(5-hydroxypent-3-enoyl)-1,4,5,6-tetrahydropyridine-3-carboxylate (8)<smiles>C=CC(=O)N1CCCCC1=O</smiles>

9

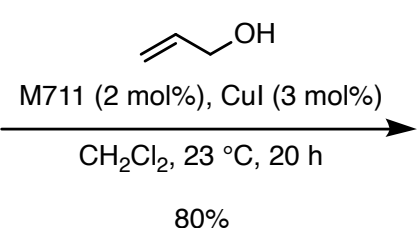

$80 \%$

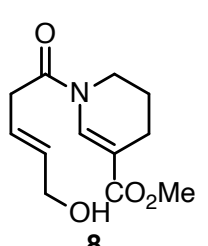

8

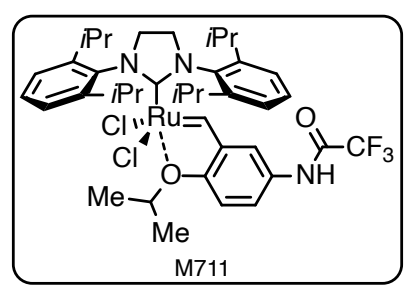

M711

A mixture of amide 9 (4.10 g, $19.6 \mathrm{mmol}, 1.0$ eq.), allyl alcohol (8.9 mL, $98 \mathrm{mmol}, 5.0$ eq.), and Cul (112 mg, $0.588 \mathrm{mmol}, 3 \mathrm{~mol} \%$ ) in dry $\mathrm{CH}_{2} \mathrm{Cl}_{2}(20 \mathrm{~mL})$ was degassed by three freeze-pumpthaw cycles. Umicore catalyst M711 (322 mg, $0.392 \mathrm{mmol}, 2 \mathrm{~mol} \%$ ) was added and the reaction mixture was stirred for $20 \mathrm{~h}$ at $23^{\circ} \mathrm{C}$. After stirring for $1 \mathrm{~h}$ with open flask, without concentration the reaction mixture was directly purified by column chromatography (nhexane/EtOAc 1:2). The allylic alcohol 8 (3.75 g, $15.7 \mathrm{mmol}, 80 \%)$ was obtained as a yellow oil.

TLC: $\mathrm{R}_{\mathrm{F}}=0.19$ (nhexane/EtOAc 1:3, UV, CAM).

4.5:1 mixture of rotamers, signals of the minor rotamer are marked with an *.

${ }^{1} \mathrm{H}-\mathrm{NMR}\left(500 \mathrm{MHz}, \mathrm{CDCl}_{3}\right): \delta=8.33^{*}(\mathrm{~s}, 1 \mathrm{H}), 7.85(\mathrm{~s}, 1 \mathrm{H}), 5.88-5.76(\mathrm{~m}, 2 \mathrm{H}), 4.16-4.14$ (m, 2H), $3.75(\mathrm{~s}, 3 \mathrm{H}), 3.67-3.64(\mathrm{~m}, 2 \mathrm{H}), 3.60^{*}(\mathrm{bs}, 2 \mathrm{H}), 3.36(\mathrm{~d}, J=6.1 \mathrm{~Hz}, 2 \mathrm{H}), 3.26^{*}$ (bs, $2 \mathrm{H}), 2.34$ (t, $J=6.2 \mathrm{~Hz}, 2 \mathrm{H}), 1.89$ (bs, $1 \mathrm{H}), 1.86-1.81$ (m, 2H) ppm.

${ }^{13}$ C-NMR $\left(126 \mathrm{MHz}, \mathrm{CDCl}_{3}\right): \delta=170.1,167.8,135.1,133.9,123.6,109.9,63.1,51.7,40.7$, 37.1, 21.4, $20.5 \mathrm{ppm}$.

IR (ATR): $\tilde{\nu}=3447,2951,1679,1620,1438,1391,1257,1227,1188,1167,1099,974,911$, $727 \mathrm{~cm}^{-1}$.

HRMS (ESI-TOF): $m / z$ calculated for $\mathrm{C}_{12} \mathrm{H}_{17} \mathrm{NO}_{4} \mathrm{Na}^{+}[\mathrm{M}+\mathrm{Na}]^{+}:$262.1050; found 262.1053. 


\section{Batch procedure:}

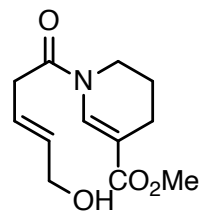

8

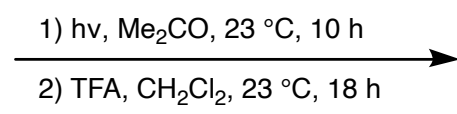

$94 \%$ (2 steps)

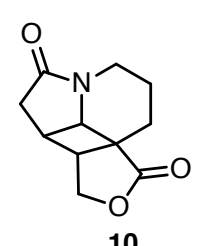

10

Allylic alcohol 8 (7.58 g, $31.7 \mathrm{mmol}, 1.0$ eq.) was dissolved in $\mathrm{Me}_{2} \mathrm{CO}(2 \mathrm{~L})$ and the resulting solution was deoxygenated by purging with argon for $30 \mathrm{~min}$. The solution was irradiated for $10 \mathrm{~h}$ at $23^{\circ} \mathrm{C}$ with a water-cooled mercury medium-pressure UV-lamp (400 W). The solvent was removed under reduced pressure and the residue was dissolved in dry $\mathrm{CH}_{2} \mathrm{Cl}_{2}$ (160 mL). Trifluoroacetic acid (4.88 mL, $63.4 \mathrm{mmol}, 2.0$ eq.) was added dropwise at $23^{\circ} \mathrm{C}$ and the reaction mixture was stirred for $18 \mathrm{~h}$ at $23^{\circ} \mathrm{C} . \mathrm{NaHCO}_{3}$ (sat. aq., $100 \mathrm{~mL}$ ) was added, the phases were separated, and the aqueous phase was extracted with $\mathrm{CH}_{2} \mathrm{Cl}_{2}(2 \times 100 \mathrm{~mL})$. The combined organic phases were dried over $\mathrm{MgSO}_{4}$ and the solvent was removed under reduced pressure to give crude lactone 10 (6.18 g, $29.8 \mathrm{mmol}, 94 \%$ over 2 steps) as a yellow solid. The crude material was used in the next step without further purification. Analytically pure $\mathbf{1 0}$ was obtained by recrystallization from THF.

TLC: not detectable on TLC.

m.p.: $132-133^{\circ} \mathrm{C}(\mathrm{THF})$.

${ }^{1} \mathrm{H}-N M R\left(500 \mathrm{MHz}, \mathrm{CDCl}_{3}\right): \delta=4.34(\mathrm{~d}, J=9.1 \mathrm{~Hz}, 1 \mathrm{H}), 4.31(\mathrm{dd}, J=9.8,5.1 \mathrm{~Hz}, 1 \mathrm{H}), 4.19-$ $4.14(\mathrm{~m}, 1 \mathrm{H}), 4.06(\mathrm{~d}, J=4.9 \mathrm{~Hz}, 1 \mathrm{H}), 2.89(\mathrm{dt}, J=7.1,5.1 \mathrm{~Hz}, 1 \mathrm{H}), 2.82(\mathrm{t}, J=5.1 \mathrm{~Hz}, 1 \mathrm{H})$, $2.75-2.68(\mathrm{~m}, 2 \mathrm{H}), 2.31(\mathrm{~d}, J=17.1 \mathrm{~Hz}, 1 \mathrm{H}), 2.03-1.95(\mathrm{~m}, 1 \mathrm{H}), 1.78-1.73(\mathrm{~m}, 2 \mathrm{H}), 1.58$ $-1.48(\mathrm{~m}, 1 \mathrm{H}) \mathrm{ppm}$.

${ }^{13} \mathrm{C}-\mathrm{NMR}\left(126 \mathrm{MHz}, \mathrm{CDCl}_{3}\right): \delta=179.1,174.1,71.1,58.8,49.2,43.5,39.1,36.4,34.9,24.9$, $21.7 \mathrm{ppm}$.

IR (ATR): $\tilde{\nu}=2944,1767,1671,1407,1297,1180,1064,980,907,724 \mathrm{~cm}^{-1}$.

HRMS (ESI-TOF): $m / z$ calculated for $\mathrm{C}_{11} \mathrm{H}_{13} \mathrm{NO}_{3} \mathrm{Na}^{+}[\mathrm{M}+\mathrm{Na}]^{+}:$230.0788; found 230.0790. 


\section{Continuous flow procedure:}

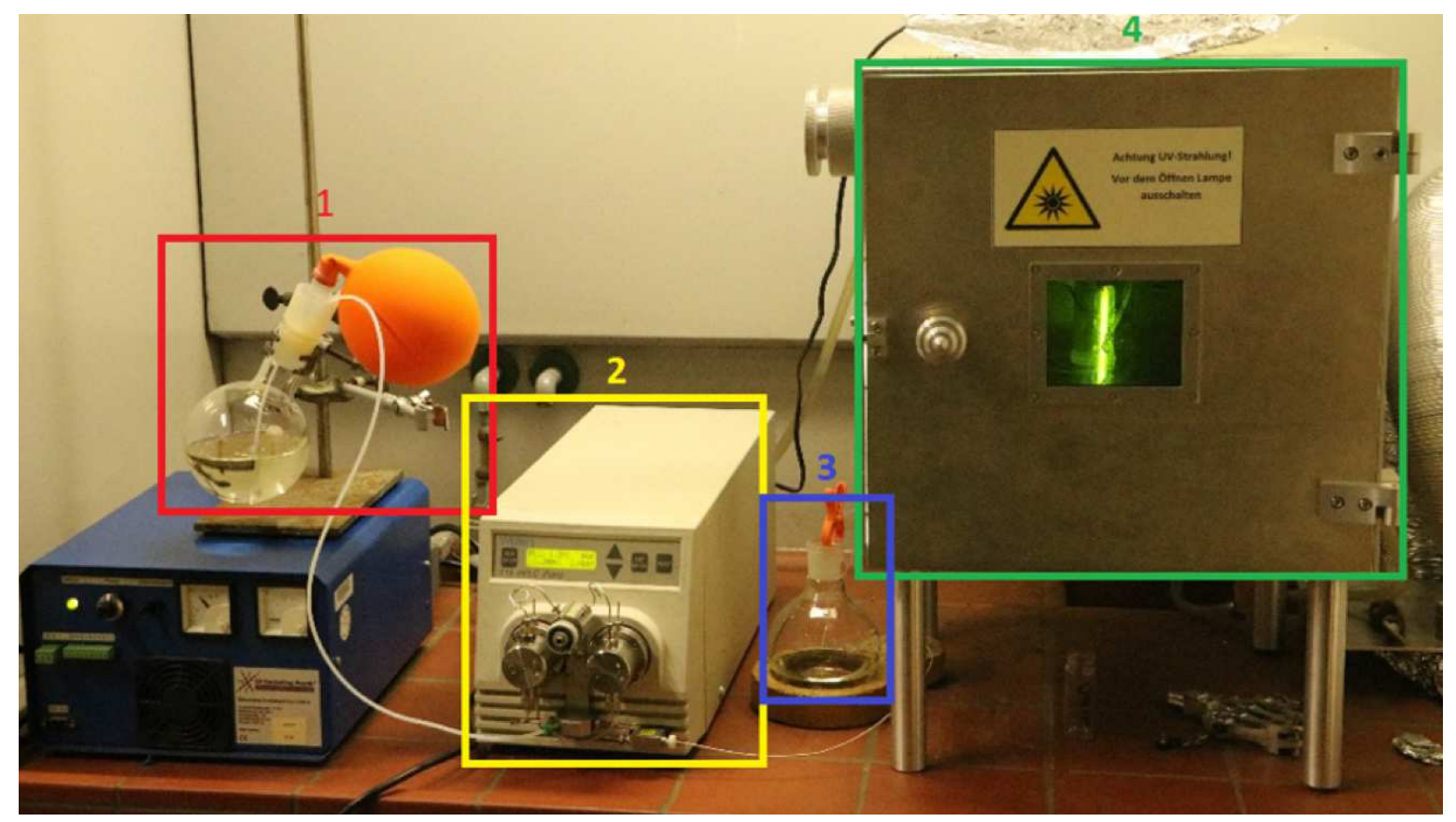

Figure S 1: Setup for the preparation of 10 in continuous flow: 1 . Solution of 8 in $\mathrm{Me}_{2}$ CO. 2 . HPLC pump. 3. Collecting flask. 4. UV-light photoreactor.

Setup: A HPLC pump was equipped with a PTFE tube (ca. $0.5 \mathrm{~m}$ ) and a PEEK capillary (ca. $0.5 \mathrm{~m}$ ). The PTFE tube was introduced to a solution of 8 in $\mathrm{Me}_{2} \mathrm{CO}$ under an atmosphere of argon (balloon). The PEEK capillary (in order to maintain a pressure of ca. 2.4 bar) was connected to the photoreactor. The photoreactor consists of a water-cooled $\mathrm{Hg}$-medium pressure UV-lamp (400 W) and a FEP tube $(2.0 \mathrm{~m}, \mathrm{~V}=1.0 \mathrm{~mL})$ that is coiled around the UV-lamp. At the end of the FEP tube, the reaction mixture is collected in a flask open to air.
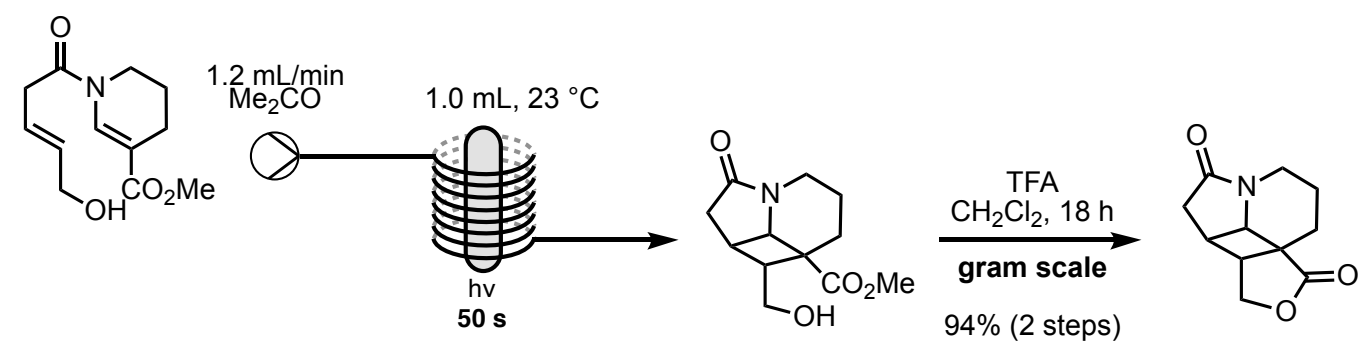

Figure S 2: Schematic of the synthesis of 10 in flow.

Allylic alcohol 8 (1.0 g, $4.2 \mathrm{mmol}, 1.0$ eq.) was dissolved in $\mathrm{Me}_{2} \mathrm{CO}(350 \mathrm{~mL})$ in a $500 \mathrm{~mL}$ round bottom flask and the solution was deoxygenated by purging with argon for $30 \mathrm{~min}$. The solution was pumped through the photoreactor at $23^{\circ} \mathrm{C}$ using the HPLC pump with a flow rate 
of $1.2 \mathrm{~mL} / \mathrm{min}$ corresponding to a residence time of $50 \mathrm{~s}$. A prerun of one residence time was discarded. Then, the reaction mixture was collected in a $500 \mathrm{~mL}$ round bottom flask. The solvent was removed under reduced pressure and the residue was dissolved in $\mathrm{dry} \mathrm{CH}_{2} \mathrm{Cl}_{2}$ (41 mL). Trifluoroacetic acid $\left(0.64 \mathrm{~mL}, 8.4 \mathrm{mmol}, 2.0\right.$ eq.) was added dropwise at $23^{\circ} \mathrm{C}$ and the reaction mixture was stirred for $18 \mathrm{~h}$ at $23^{\circ} \mathrm{C}$. $\mathrm{NaHCO}_{3}$ (sat. aq., $100 \mathrm{~mL}$ ) was added, the phases were separated, and the aqueous phase was extracted with $\mathrm{CH}_{2} \mathrm{Cl}_{2}(2 \times 100 \mathrm{~mL})$. The combined organic phases were dried over $\mathrm{MgSO}_{4}$ and the solvent was removed under reduced pressure to give crude lactone 10 (0.82 g, $3.9 \mathrm{mmol}, 94 \%$ over 2 steps) as a yellow solid.

\section{(6-Oxohexahydro-1H-cyclobuta[hi]indolizine-1,1a(2H)-diyl)bis(methylene)}

\section{dimethanesulfonate (S5)}
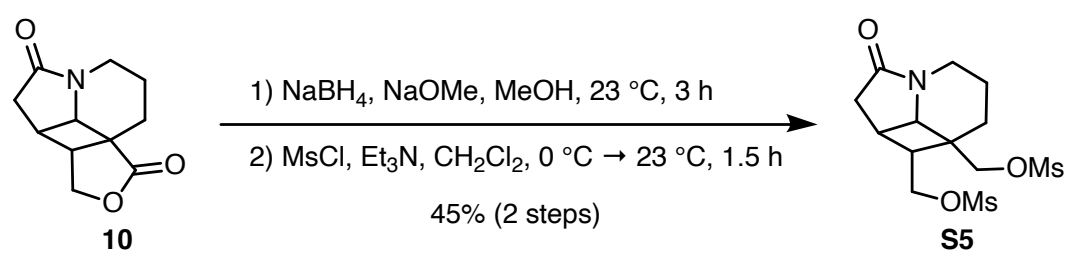

To a solution of crude lactone 10 (6.18 g, $29.8 \mathrm{mmol}, 1.0$ eq.) and $\mathrm{NaOMe}(0.5 \mathrm{M}$ in $\mathrm{MeOH}$, $3.0 \mathrm{~mL}, 1.5 \mathrm{mmol}, 5 \mathrm{~mol} \%)$ in dry $\mathrm{MeOH}(20 \mathrm{~mL}), \mathrm{NaBH}_{4}(2.25 \mathrm{~g}, 59.6 \mathrm{mmol}, 2.0$ eq.) was added portionwise over 5 min at $23^{\circ} \mathrm{C}$. After stirring for $3 \mathrm{~h}, \mathrm{HCl}\left(1 \mathrm{M} \mathrm{in} \mathrm{H}_{2} \mathrm{O}, 50 \mathrm{~mL}\right)$ was added and the resulting solution was extracted with $\mathrm{CHCl}_{3} / \mathrm{iPrOH}(4: 1,10 \times 30 \mathrm{~mL})$. The combined organic phases were dried over $\mathrm{Na}_{2} \mathrm{SO}_{4}$ and the solvent was removed under reduced pressure. The crude diol was dissolved in dry $\mathrm{CH}_{2} \mathrm{Cl}_{2}(190 \mathrm{~mL})$ and the resulting solution was cooled to $0^{\circ} \mathrm{C}$. $\mathrm{Et}_{3} \mathrm{~N}(24.8 \mathrm{~mL}, 179 \mathrm{mmol}, 6.0$ eq.) and methanesulfonyl chloride $(9.22 \mathrm{~mL}, 119 \mathrm{mmol}$, 4.0 eq.) were added sequentially and the reaction mixture was stirred for 20 min at $0^{\circ} \mathrm{C}$ and then $1 \mathrm{~h}$ at $23^{\circ} \mathrm{C}$. $\mathrm{NaHCO}_{3}$ (sat. aq., $200 \mathrm{~mL}$ ) was added and the phases were separated. The aqueous phase was extracted with $\mathrm{CH}_{2} \mathrm{Cl}_{2}(3 \times 150 \mathrm{~mL})$ and the combined organic phases were dried over $\mathrm{MgSO}_{4}$. The solvent was removed under reduced pressure and the crude product was purified by column chromatography $\left(\mathrm{CH}_{2} \mathrm{Cl}_{2} / \mathrm{MeOH} 50: 1 \rightarrow 19: 1\right)$ to give bismesylate S5 (4.96 g, $13.5 \mathrm{mmol}, 45 \%$ over 2 steps) as a colorless oil.

TLC: $\mathrm{R}_{\mathrm{F}}=0.27\left(\mathrm{CH}_{2} \mathrm{Cl}_{2} / \mathrm{MeOH} 19: 1, \mathrm{KMnO}_{4}\right)$.

${ }^{1} \mathrm{H}-\mathrm{NMR}\left(500 \mathrm{MHz}, \mathrm{CDCl}_{3}\right): \delta=4.39(\mathrm{dd}, J=10.5,8.1 \mathrm{~Hz}, 1 \mathrm{H}), 4.32-4.29(\mathrm{~m}, 3 \mathrm{H}), 4.09$ (dt, $J=13.4,4.1 \mathrm{~Hz}, 1 \mathrm{H}), 3.87(\mathrm{~d}, J=5.7 \mathrm{~Hz}, 1 \mathrm{H}), 3.10(\mathrm{~s}, 3 \mathrm{H}), 3.03(\mathrm{~s}, 3 \mathrm{H}), 2.86(\mathrm{dt}, J=7.7$, 
$6.1 \mathrm{~Hz}, 1 \mathrm{H}), 2.70-2.59(\mathrm{~m}, 3 \mathrm{H}), 2.25(\mathrm{~d}, J=17.0 \mathrm{~Hz}, 1 \mathrm{H}), 1.72-1.55(\mathrm{~m}, 4 \mathrm{H}) \mathrm{ppm}$.

${ }^{13}$ C-NMR $\left(151 \mathrm{MHz}, \mathrm{CDCl}_{3}\right): \delta=174.8,71.1,67.3,56.7,43.2,41.3,39.0,37.7,37.6,36.5$, 32.3, 27.4, $20.7 \mathrm{ppm}$.

IR (ATR): $\tilde{\nu}=2938,1681,1407,1348,1172,950,833 \mathrm{~cm}^{-1}$.

HRMS (ESI-TOF): $m / z$ calculated for $\mathrm{C}_{13} \mathrm{H}_{21} \mathrm{NO}_{7} \mathrm{~S}_{2} \mathrm{Na}^{+}[\mathrm{M}+\mathrm{Na}]^{+}:$390.0652; found 390.0645 .

Hexahydro-1 H,9H-thieno[3',4':1,4]cyclobuta[1,2,3-hi]indolizin-5(4' $\left.{ }^{1} H\right)$-one (S6)

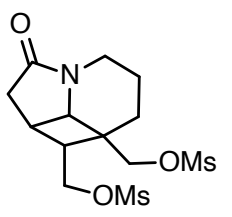

S5

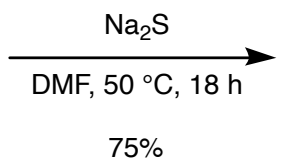

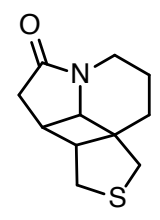

S6

A solution of bismesylate $\mathbf{S} 5$ (4.96 g, $13.5 \mathrm{mmol}, 1.0 \mathrm{eq}$.) and $\mathrm{Na}_{2} \mathrm{~S}$ (2.63 g, $33.8 \mathrm{mmol}, 2.5 \mathrm{eq}$.) in dry DMF $(270 \mathrm{~mL})$ was stirred at $50^{\circ} \mathrm{C}$ for $18 \mathrm{~h}$. After cooling to $23^{\circ} \mathrm{C}$, the reaction mixture was filtered and the filtrate was concentrated under reduced pressure. $\mathrm{H}_{2} \mathrm{O}(500 \mathrm{~mL})$ was added and the aqueous phase was extracted with $\mathrm{CH}_{2} \mathrm{Cl}_{2}(4 \times 200 \mathrm{~mL})$. The combined organic phases were dried over $\mathrm{MgSO}_{4}$ and the solvent was removed under reduced pressure. The crude product was purified by column chromatography (nhexane/EtOAc 1:2) to give thiolane S6 (2.12 g, $10.2 \mathrm{mmol}, 75 \%)$ as a colorless solid.

TLC: $\mathrm{R}_{\mathrm{F}}=0.18\left(\right.$ nhexane/EtOAc 1:2, $\left.\mathrm{KMnO}_{4}\right)$.

m.p.: $66-67^{\circ} \mathrm{C}$ (EtOAc).

${ }^{1} \mathrm{H}-\mathrm{NMR}\left(500 \mathrm{MHz}, \mathrm{CDCl}_{3}\right): \delta=4.09(\mathrm{dt}, J=12.7,5.0 \mathrm{~Hz}, 1 \mathrm{H}), 3.75(\mathrm{~d}, J=5.8 \mathrm{~Hz}, 1 \mathrm{H}), 2.93$ (dd, $J=12.1,5.0 \mathrm{~Hz}, 1 \mathrm{H}$ ), 2.85 (d, $J=12.3 \mathrm{~Hz}, 1 \mathrm{H}$ ), 2.80 (dt, $J=8.0,5.5 \mathrm{~Hz}, 1 \mathrm{H}$ ), $2.75-2.71$ $(\mathrm{m}, 1 \mathrm{H}), 2.71-2.68(\mathrm{~m}, 2 \mathrm{H}), 2.67-2.65(\mathrm{~m}, 1 \mathrm{H}), 2.64-2.60(\mathrm{~m}, 1 \mathrm{H}), 2.24(\mathrm{~d}, J=17.2 \mathrm{~Hz}$, $1 \mathrm{H}), 1.71-1.52(\mathrm{~m}, 4 \mathrm{H}) \mathrm{ppm}$.

${ }^{13}$ C-NMR $\left(126 \mathrm{MHz}, \mathrm{CDCl}_{3}\right): \delta=175.8,60.8,53.3,52.6,42.0,39.2,38.4,36.8,32.5,30.5$, $23.0 \mathrm{ppm}$.

IR (ATR): $\tilde{\nu}=2914,1684,1444,1400,1301,1213,1150,916 \mathrm{~cm}^{-1}$.

HRMS (ESI-TOF): $m / z$ calculated for $\mathrm{C}_{11} \mathrm{H}_{15} \mathrm{NOSNa}^{+}[\mathrm{M}+\mathrm{Na}]^{+}:$232.0767; found 232.0764 . 
Hexahydro-1 $H, 9 H$-thieno[3',4':1,4]cyclobuta[1,2,3-hi]indolizin-5(4'H)-one 8-oxide (7)

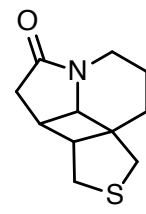

S6

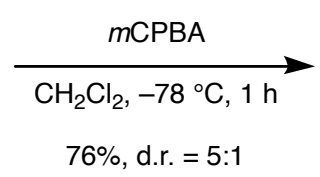

$76 \%$, d.r. $=5: 1$

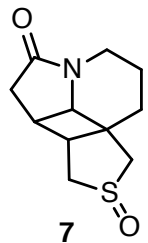

To a solution of thiolane $\mathbf{S} 6\left(2.12 \mathrm{~g}, 10.2 \mathrm{mmol}, 1.0\right.$ eq.) in $\mathrm{CH}_{2} \mathrm{Cl}_{2}(100 \mathrm{~mL})$ at $-78^{\circ} \mathrm{C}$ was added meta-chloroperoxybenzoic acid (70\% w/w, $2.51 \mathrm{~g}, 10.2 \mathrm{mmol}, 1.0$ eq.) portionwise. The reaction mixture was stirred for $1 \mathrm{~h}$ at $-78^{\circ} \mathrm{C}$ and then poured into a mixture of $\mathrm{NaOH}(1 \mathrm{M}$ aq., $50 \mathrm{~mL}$ ) and $\mathrm{Na}_{2} \mathrm{~S}_{2} \mathrm{O}_{3}$ (sat. aq., $50 \mathrm{~mL}$ ). The organic phase was separated and the aqueous phase was extracted with $\mathrm{CH}_{2} \mathrm{Cl}_{2}(4 \times 50 \mathrm{~mL})$. The combined organic phases were dried over $\mathrm{MgSO}_{4}$ and the solvent was removed under reduced pressure. Purification by column chromatography $\left(\mathrm{CH}_{2} \mathrm{Cl}_{2} / \mathrm{MeOH} 50: 1 \rightarrow 19: 1\right)$ gave sulfoxide $7(1.74 \mathrm{~g}, 7.74 \mathrm{mmol}, 76 \%$, d.r. = 5:1 [determined by integration of signals $\delta=3.79 \mathrm{ppm}$ and $\delta=3.66 \mathrm{ppm}$ in ${ }^{1} \mathrm{H}-\mathrm{NMR}$ ]) as a colorless solid.

TLC: $\mathrm{R}_{\mathrm{F}}=0.40\left(\mathrm{CH}_{2} \mathrm{Cl}_{2} / \mathrm{MeOH} 19: 1, \mathrm{KMnO}_{4}\right)$.

m.p.: $122-123^{\circ} \mathrm{C}\left(\mathrm{CH}_{2} \mathrm{Cl}_{2} / \mathrm{MeOH}\right)$.

Significant signals of the minor diastereoisomer are marked with *.

${ }^{1} \mathrm{H}-\mathrm{NMR}\left(500 \mathrm{MHz}, \mathrm{CDCl}_{3}\right): \delta=4.13-4.08(\mathrm{~m}, 1 \mathrm{H}), 3.79(\mathrm{~d}, J=5.3 \mathrm{~Hz}, 1 \mathrm{H}), 3.66^{*}(\mathrm{dt}, J=7.9$, $5.8 \mathrm{~Hz}, 1 \mathrm{H}), 3.35^{\star}(\mathrm{dd}, J=15.2,1.8 \mathrm{~Hz}, 1 \mathrm{H}), 3.24-3.12(\mathrm{~m}, 2 \mathrm{H}), 3.08-2.96(\mathrm{~m}, 3 \mathrm{H}), 2.83$ $-2.76^{*}(\mathrm{~m}, 1 \mathrm{H}), 2.72-2.60(\mathrm{~m}, 2 \mathrm{H}), 2.49(\mathrm{dt}, J=7.0,5.7 \mathrm{~Hz}, 1 \mathrm{H}), 2.27(\mathrm{~d}, J=17.1 \mathrm{~Hz}, 1 \mathrm{H})$, 2.23* (d, $J=17.4 \mathrm{~Hz}, 1 \mathrm{H}), 2.17(\mathrm{dt}, J=14.5,4.2 \mathrm{~Hz}, 1 \mathrm{H}), 1.78-1.72(\mathrm{~m}, 1 \mathrm{H}), 1.68-1.56(\mathrm{~m}$, 2H) ppm.

${ }^{13}$ C-NMR $\left(126 \mathrm{MHz}, \mathrm{CDCl}_{3}\right): \delta=175.4^{*}, 174.5,64.0^{*}, 61.9,60.6^{*}, 60.0,59.1,57.1^{*}, 53.8^{*}$, 53.3, 52.3* ${ }^{\star}, 47.6,39.4,38.9^{\star}, 36.9^{\star}, 36.7,36.2,33.7^{\star}, 32.3,31.2^{*}, 22.8^{\star}, 22.6$ ppm.

IR (ATR): $\tilde{\nu}=2927,1667,1409,1302,1262,1025,999 \mathrm{~cm}^{-1}$.

HRMS (ESI-TOF): $m / z$ calculated for $\mathrm{C}_{11} \mathrm{H}_{15} \mathrm{NO}_{2} \mathrm{SNa}^{+}[\mathrm{M}+\mathrm{Na}]^{+}:$248.0716; found 248.0718. 


\section{9-Chlorohexahydro-1 $H, 9 H$-thieno[3',4':1,4]cyclobuta[1,2,3-hi]indolizin-5(4' $H)$-one 8-oxide (S7)}
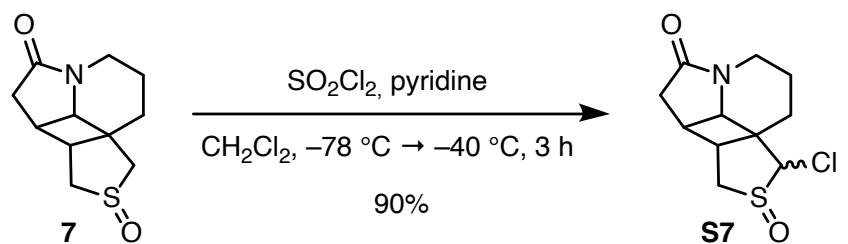

A solution of sulfoxide $\mathbf{S 7}(1.74 \mathrm{~g}, 7.74 \mathrm{mmol}, 1.0$ eq.) and pyridine $(1.31 \mathrm{~mL}, 16.3 \mathrm{mmol}$, 2.1 eq.) in dry $\mathrm{CH}_{2} \mathrm{Cl}_{2}(80 \mathrm{~mL})$ was cooled to $-78^{\circ} \mathrm{C}$ and $\mathrm{SO}_{2} \mathrm{Cl}_{2}(0.66 \mathrm{~mL}, 8.1 \mathrm{mmol}, 1.05$ eq.) in dry $\mathrm{CH}_{2} \mathrm{Cl}_{2}(6 \mathrm{~mL})$ was added over a period of $5 \mathrm{~min}$. The reaction mixture was allowed to warm to $-40^{\circ} \mathrm{C}$ over $3 \mathrm{~h}, \mathrm{HCl}\left(1 \mathrm{M}\right.$ in $\mathrm{H}_{2} \mathrm{O}, 100 \mathrm{~mL}$ ) was added, and the phases were separated. The aqueous phase was extracted with $\mathrm{CH}_{2} \mathrm{Cl}_{2}(3 \times 50 \mathrm{~mL})$, the combined organic phases were dried over $\mathrm{MgSO}_{4}$, and the solvent was removed under reduced pressure. The crude product was purified by column chromatography $\left(\mathrm{CH}_{2} \mathrm{Cl}_{2} / \mathrm{MeOH} 19: 1\right)$ to give $\alpha$-chlorinated sulfoxide $\mathbf{S 7}(1.81 \mathrm{~g}, 6.97 \mathrm{mmol}, 90 \%$, complex mixture of diastereoisomers) as a colorless oil.

TLC: $\mathrm{R}_{\mathrm{F}}=0.33\left(\mathrm{CH}_{2} \mathrm{Cl}_{2} / \mathrm{MeOH} 19: 1, \mathrm{KMnO}_{4}\right)$.

Complex mixture of diastereoisomers, significant signals of minor isomers are marked with an *. ${ }^{1} \mathrm{H}-\mathrm{NMR}\left(500 \mathrm{MHz}, \mathrm{CDCl}_{3}\right): \delta=4.91^{*}(\mathrm{~s}, 1 \mathrm{H}), 4.68(\mathrm{~s}, 1 \mathrm{H}), 4.41$ (d, J = 6.0 Hz, 1H), 4.09 $4.03(\mathrm{~m}, 2 \mathrm{H}), 3.84^{*}(\mathrm{~d}, J=5.9 \mathrm{~Hz}, 1 \mathrm{H}), 3.56(\mathrm{dt}, J=8.2,5.8 \mathrm{~Hz}, 1 \mathrm{H}), 3.26(\mathrm{~m}, 1 \mathrm{H}), 3.11^{*}(\mathrm{dd}$, $J=13.9,2.9 \mathrm{~Hz}, 1 \mathrm{H}$ ), 3.01 (ddd, $J=14.5,6.6,1.0 \mathrm{~Hz}, 1 \mathrm{H}$ ), 2.95* (ddd, $J=8.2,5.2,2.8 \mathrm{~Hz}$, $1 \mathrm{H}), 2.86-2.82^{*}(\mathrm{~m}, 1 \mathrm{H}), 2.82-2.77(\mathrm{~m}, 1 \mathrm{H}), 2.67-2.62(\mathrm{~m}, 1 \mathrm{H}), 2.32^{*}(\mathrm{~d}, J=17.1 \mathrm{~Hz}, 1 \mathrm{H})$, $2.22(\mathrm{~d}, J=17.4 \mathrm{~Hz}, 1 \mathrm{H}), 1.84-1.75(\mathrm{~m} 2 \mathrm{H}), 1.74-1.65(\mathrm{~m}, 2 \mathrm{H}) \mathrm{ppm}$.

${ }^{13} \mathrm{C}-N M R\left(151 \mathrm{MHz}, \mathrm{CDCl}_{3}\right): \delta=175.2,174.8^{\star}, 81.5,77.7^{\star}, 57.7^{\star}, 56.7,55.4^{\star}, 54.2^{\star}, 53.5$, 53.3, 51.1, 47.7*, 38.7* $38.3,36.8,36.6^{\star}, 36.0^{\star}, 34.2,30.7,26.9^{\star}, 21.6,20.6^{\star} \mathrm{ppm}$.

IR (ATR): $\tilde{\nu}=2927,1678,1403,1287,1199,1054,727 \mathrm{~cm}^{-1}$.

HRMS (ESI-TOF): $m / z$ calculated for $\mathrm{C}_{11} \mathrm{H}_{14} \mathrm{CINO}_{2} \mathrm{SNa}^{+}[\mathrm{M}+\mathrm{Na}]^{+}: 282.0326$; found 282.0338 . 


\section{2,3,6a,6b-Tetrahydro-1 $H, 4^{1} H$-cyclobuta[1,4]cyclobuta[1,2,3-hi]indolizin-5(6H)-one (6)}

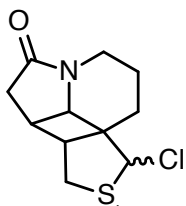

s7 "o

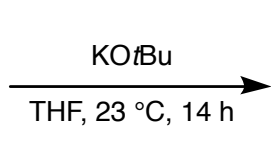

$43 \%$

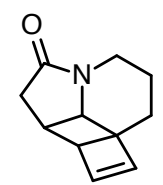

6

To a solution of $\alpha$-chlorinated sulfoxide $\mathbf{S} 7(1.81 \mathrm{~g}, 6.97 \mathrm{mmol}, 1.0$ eq.) in dry THF (230 mL), $\mathrm{KOtBu}\left(3.91 \mathrm{~g}, 34.8 \mathrm{mmol}, 5.0\right.$ eq.) was added in one portion at $23^{\circ} \mathrm{C}$. After stirring the dark red reaction mixture for $14 \mathrm{~h}$ at $23^{\circ} \mathrm{C}, \mathrm{HCl}\left(1 \mathrm{M}\right.$ in $\left.\mathrm{H}_{2} \mathrm{O}, 200 \mathrm{~mL}\right)$ was added. The organic phase was separated and the aqueous phase was extracted with EtOAc $(3 \times 100 \mathrm{~mL})$. The combined organic phases were washed sequentially with $\mathrm{NaHCO}_{3}$ (sat. aq., $2 \times 200 \mathrm{~mL}$ ) and brine (sat., $200 \mathrm{~mL}$ ), dried over $\mathrm{MgSO}_{4}$, and the solvent was removed under reduced pressure. Purification by column chromatography (nhexane/EtOAc 1:2) gave cyclobutene 6 (526 mg, 3.00 mmol, $43 \%)$ as a yellow oil.

TLC: $\mathrm{R}_{\mathrm{F}}=0.38$ (nhexane/EtOAc 1:2, $\mathrm{KMnO}_{4}$ ).

${ }^{1} \mathrm{H}-\mathrm{NMR}\left(500 \mathrm{MHz}, \mathrm{CDCl}_{3}\right): \delta=6.49(\mathrm{~d}, J=2.6 \mathrm{~Hz}, 1 \mathrm{H}), 6.10(\mathrm{t}, J=2.8 \mathrm{~Hz}, 1 \mathrm{H}), 4.10$ (dddd, $J=13.1,4.3,2.8,1.5 \mathrm{~Hz}, 1 \mathrm{H}), 3.93(\mathrm{~d}, J=4.7 \mathrm{~Hz}, 1 \mathrm{H}), 2.91-2.89(\mathrm{~m}, 1 \mathrm{H}), 2.70(\mathrm{dd}, J=17.4$, 8.2 Hz, 1H), 2.60 (tdd, $J=12.8,3.3,1.0 \mathrm{~Hz}, 1 \mathrm{H}$ ), 2.49 (ddd, $J=8.2,4.6,1.9 \mathrm{~Hz}, 1 \mathrm{H}$ ), 2.27 (d, $J=17.4 \mathrm{~Hz}, 1 \mathrm{H}$ ), 1.85 (ddd, $J=15.0,12.2,5.7 \mathrm{~Hz}, 1 \mathrm{H}$ ), 1.75 (dddd, $J=15.0,4.5,2.8,1.4 \mathrm{~Hz}$, $1 \mathrm{H}), 1.61-1.47(\mathrm{~m}, 2 \mathrm{H}) \mathrm{ppm}$.

${ }^{13} \mathrm{C}-N M R\left(126 \mathrm{MHz}, \mathrm{CDCl}_{3}\right): \delta=175.0,142.4,140.5,58.9,55.9,52.4,39.9,36.5,34.6,27.1$, $23.2 \mathrm{ppm}$.

IR (ATR): $\tilde{\nu}=2921,1682,1445,1397,1293,1231,1162,1115,913,846,768,729 \mathrm{~cm}^{-1}$. HRMS (ESI-TOF): $m / z$ calculated for $\mathrm{C}_{11} \mathrm{H}_{13} \mathrm{NONa}^{+}[\mathrm{M}+\mathrm{Na}]^{+}:$198.0889; found 198.0893 . 


\section{$3^{1}, 5,6,9 a-T e t r a h y d r o-4 H-p y r r o l o[3,2,1-i j] q u i n o l i n-2(1 H)$-one $(5)$}

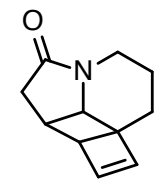

6

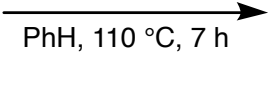

$82 \%$

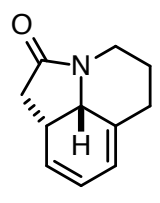

5

A solution of cyclobutene $6(526 \mathrm{mg}, 3.00 \mathrm{mmol}, 1.0$ eq.) in benzene $(150 \mathrm{~mL})$ in a pressure tube was heated for $7 \mathrm{~h}$ to $110^{\circ} \mathrm{C}$. The mixture was allowed to cool to $23^{\circ} \mathrm{C}$ and the solvent was removed under reduced pressure. The crude product was purified by column chromatography ( $n$ hexane/EtOAc 1:2) to give diene 5 (433 mg, $2.47 \mathrm{mmol}, 82 \%$ ) as a yellow solid and reisolated cyclobutene 6 (22.4 mg, $0.128 \mathrm{mmol}, 4 \%$ ).

TLC: $\mathrm{R}_{\mathrm{F}}=0.29$ (nhexane/EtOAc 1:3, UV, CAM, $\mathrm{KMnO}_{4}$ ).

m.p.: $62-63^{\circ} \mathrm{C}(\mathrm{EtOAc})$.

${ }^{1} \mathrm{H}-\mathrm{NMR}\left(500 \mathrm{MHz}, \mathrm{CDCl}_{3}\right): \delta=5.74(\mathrm{dd}, J=9.9,5.2 \mathrm{~Hz}, 1 \mathrm{H}), 5.72-5.68(\mathrm{~m}, 1 \mathrm{H}), 5.54$ - $5.52(\mathrm{~m}, 1 \mathrm{H}), 4.32(\mathrm{~d}, J=12.0 \mathrm{~Hz}, 1 \mathrm{H}), 4.23$ (ddt, $J=13.1,4.9,1.7 \mathrm{~Hz}, 1 \mathrm{H}), 3.16$ (ddd, $J=21.5,10.0,4.7 \mathrm{~Hz}, 1 \mathrm{H}), 2.82-2.77(\mathrm{~m}, 1 \mathrm{H}), 2.73(\mathrm{ddd}, J=17.0,10.2,1.4 \mathrm{~Hz}, 1 \mathrm{H}), 2.41$ (ddt, $J=13.9,4.3,2.0 \mathrm{~Hz}, 1 \mathrm{H}$ ), 2.29 (dd, $J=16.9,9.5 \mathrm{~Hz}, 1 \mathrm{H}$ ), 2.18 (ddd, $J=6.5,9.4,4.0 \mathrm{~Hz}$, $1 \mathrm{H}), 1.78-1.73(\mathrm{~m}, 1 \mathrm{H}), 1.45$ (qt, $J=13.0,4.4 \mathrm{~Hz}, 1 \mathrm{H}) \mathrm{ppm}$.

${ }^{13}$ C-NMR $\left(126 \mathrm{MHz}, \mathrm{CDCl}_{3}\right): \delta=173.2,132.9,126.3,121.6,116.1,58.7,40.7,39.8,32.0$, 29.5, $26.1 \mathrm{ppm}$.

IR (ATR): $\tilde{\nu}=2928,1675,1417,1258,1140,990,868,734 \mathrm{~cm}^{-1}$.

HRMS (ESI-TOF): $m / z$ calculated for $\mathrm{C}_{11} \mathrm{H}_{13} \mathrm{NONa}^{+}[\mathrm{M}+\mathrm{Na}]^{+}:$198.0889; found 198.0899.

Table S 1: Opimization of reaction conditions

\begin{tabular}{ccc} 
entry & conditions & yield \\
\hline \hline 1 & $\mathrm{PhH}, 80^{\circ} \mathrm{C}$ & no reaction \\
2 & $\mathrm{PhH}, 110^{\circ} \mathrm{C}$ & $82 \%$ \\
3 & $\mathrm{PhH}, 120^{\circ} \mathrm{C}$ & $72 \%$ \\
4 & $\mathrm{PhMe}, 110^{\circ} \mathrm{C}$ & $65 \%$ \\
\hline
\end{tabular}




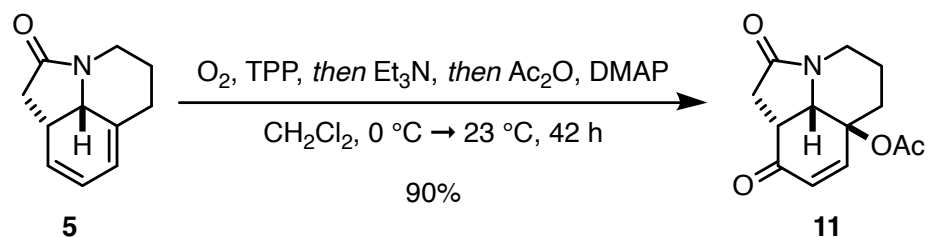

A solution of diene 5 (103 mg, $0.587 \mathrm{mmol}, 1.0$ eq.) and tetraphenylporphyrin (3.6 mg, $5.9 \mu \mathrm{mol}$, $1 \mathrm{~mol} \%)$ in dry $\mathrm{CH}_{2} \mathrm{Cl}_{2}(20 \mathrm{~mL})$ was cooled to $0^{\circ} \mathrm{C}$ and oxygen was bubbled through the solution while the reaction mixture was irradiated with white light $(300 \mathrm{~W})$ for $30 \mathrm{~min}$ at this temperature. After purging with argon for $10 \mathrm{~min}, \mathrm{Et}_{3} \mathrm{~N}\left(0.162 \mathrm{~mL}, 1.17 \mathrm{mmol}, 2.0\right.$ eq.) was added at $23^{\circ} \mathrm{C}$ and the mixture was stirred for $18 \mathrm{~h}$ at $23^{\circ} \mathrm{C}$. Additional $\mathrm{Et}_{3} \mathrm{~N}(0.162 \mathrm{~mL}, 1.17 \mathrm{mmol}, 2.0 \mathrm{eq}$ ), $\mathrm{Ac}_{2} \mathrm{O}$ (0.195 mL, $1.76 \mathrm{mmol}, 3.0$ eq.), and 4-dimethylaminopyridine (7.2 mg, 58 umol, 0.1 eq.) were added and the solution was stirred for $24 \mathrm{~h}$ at $23^{\circ} \mathrm{C}$. $\mathrm{HCl}(1 \mathrm{M} \mathrm{aq} ., 20 \mathrm{~mL})$ was added, the organic phase was separated, and the aqueous phase was extracted with $\mathrm{CH}_{2} \mathrm{Cl}_{2}(3 \times 20 \mathrm{~mL})$. The combined organic phases were dried over $\mathrm{MgSO}_{4}$, the solvent was removed under reduced pressure, and the crude product was purified by column chromatography $\left(\mathrm{CH}_{2} \mathrm{Cl}_{2} / \mathrm{MeOH}\right.$ $100: 1 \rightarrow 50: 1$ ) to give enone 11 (132 $\mathrm{mg}, 0.528 \mathrm{mmol}, 90 \%$ ) as an off-white solid.

TLC: $\mathrm{R}_{\mathrm{F}}=0.44\left(\mathrm{CH}_{2} \mathrm{Cl}_{2} / \mathrm{MeOH} 19: 1, \mathrm{UV}, \mathrm{CAM}\right)$.

m.p.: $101-102^{\circ} \mathrm{C}\left(\mathrm{CH}_{2} \mathrm{Cl}_{2} / \mathrm{MeOH}\right)$.

${ }^{1} \mathrm{H}-N M R\left(500 \mathrm{MHz}, \mathrm{CDCl}_{3}\right): \delta=7.13-7.11(\mathrm{~m}, 1 \mathrm{H}), 6.18(\mathrm{~d}, J=10.2 \mathrm{~Hz}, 1 \mathrm{H}), 4.01$ (dd, $J=13.2,5.1 \mathrm{~Hz}, 1 \mathrm{H}), 3.92(\mathrm{dd}, J=5.6,2.0 \mathrm{~Hz}, 1 \mathrm{H}), 3.20-3.17(\mathrm{~m}, 1 \mathrm{H}), 2.95(\mathrm{dt}, J=13.0$, $3.5 \mathrm{~Hz}, 1 \mathrm{H}), 2.70-2.63(\mathrm{~m}, 3 \mathrm{H}), 2.01(\mathrm{~s}, 3 \mathrm{H}), 1.88-1.82(\mathrm{~m}, 1 \mathrm{H}), 1.71(\mathrm{td}, J=13.5,3.6 \mathrm{~Hz}$, $1 \mathrm{H}), 1.44-1.35(\mathrm{~m}, 1 \mathrm{H}) \mathrm{ppm}$.

${ }^{13} \mathrm{C}-\mathrm{NMR}\left(126 \mathrm{MHz}, \mathrm{CDCl}_{3}\right): \delta=197.1,172.8,169.4,146.5,131.7,73.6,61.5,39.8,39.6$, 35.3, 33.4, 21.5, $20.7 \mathrm{ppm}$.

IR (ATR): $\tilde{\nu}=2948,2871,1739,1699,1679,1417,1370,1238,1152,1039 \mathrm{~cm}^{-1}$.

HRMS (ESI-TOF): $m / z$ calculated for $\mathrm{C}_{13} \mathrm{H}_{15} \mathrm{NO}_{4} \mathrm{Na}^{+}[\mathrm{M}+\mathrm{Na}]^{+}:$272.0893; found 272.0905. 


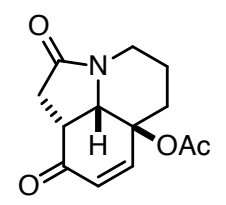

11

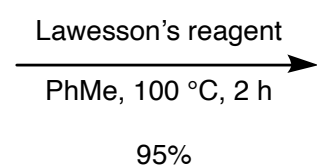

$95 \%$

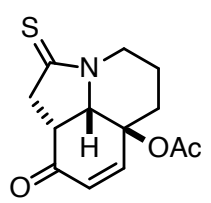

S8

A solution of enone 11 (50 mg, $0.20 \mathrm{mmol}, 1.0$ eq.) and Lawesson's reagent ( $49 \mathrm{mg}, 0.12 \mathrm{mmol}$, 0.6 eq.) in dry toluene $(2 \mathrm{~mL})$ was stirred for $2 \mathrm{~h}$ at $100^{\circ} \mathrm{C}$. The reaction mixture was allowed to cool to $23^{\circ} \mathrm{C}$, the solvent was removed under reduced pressure, and the crude product was purified by column chromatography (nhexane/EtOAc $2: 1 \rightarrow 1: 1)$ to give thiolactam $\mathbf{S} 8$ (50.5 mg, $0.190 \mathrm{mmol}, 95 \%)$ as a colorless solid.

TLC: $\mathrm{R}_{\mathrm{F}}=0.33$ (nhexane/EtOAc 1:1, UV, CAM).

m.p.: $139-140^{\circ} \mathrm{C}(\mathrm{EtOAc})$.

${ }^{1} \mathrm{H}-\mathrm{NMR}\left(500 \mathrm{MHz}, \mathrm{CDCl}_{3}\right): \delta=7.13(\mathrm{dd}, J=10.2,1.9 \mathrm{~Hz}, 1 \mathrm{H}), 6.20(\mathrm{~d}, J=10.2 \mathrm{~Hz}, 1 \mathrm{H}), 4.71$ (ddt, $J=13.9,5.2,1.7 \mathrm{~Hz}, 1 \mathrm{H}$ ), 4.17 (d, $J=5.8 \mathrm{~Hz}, 1 \mathrm{H}$ ), 3.35 (d, $J=18.1 \mathrm{~Hz}, 1 \mathrm{H}$ ), 3.26 (ddd, $J=9.6,5.9,1.0 \mathrm{~Hz}, 1 \mathrm{H}), 3.15(\mathrm{ddd}, J=18.1,9.5,2.2 \mathrm{~Hz}, 1 \mathrm{H}), 3.01-2.97(\mathrm{~m}, 1 \mathrm{H}), 2.88-2.82$ (m, 1H), 2.03 (s, 3H), 1.98 (dqd, $J=14.4,3.8,2.0 \mathrm{~Hz}, 1 \mathrm{H}), 1.80$ (td, $J=13.5,3.6 \mathrm{~Hz}, 1 \mathrm{H}), 1.57$ $-1.47(\mathrm{~m}, 1 \mathrm{H}) \mathrm{ppm}$.

${ }^{13}$ C-NMR $\left(126 \mathrm{MHz}, \mathrm{CDCl}_{3}\right): \delta=200.6,196.1,169.3,146.3,132.1,73.5,67.8,47.6,44.7$, 40.9, 32.6, 21.5, $20.8 \mathrm{ppm}$.

IR (ATR): $\tilde{\nu}=2933,2869,1735,1676,1473,1445,1368,1308,1231,1154,1053,1030$, $874 \mathrm{~cm}^{-1}$.

HRMS (ESI-TOF): $m / z$ calculated for $\mathrm{C}_{13} \mathrm{H}_{15} \mathrm{NO}_{3} \mathrm{SNa}^{+}[\mathrm{M}+\mathrm{Na}]^{+}:$288.0665; found 288.0666. 


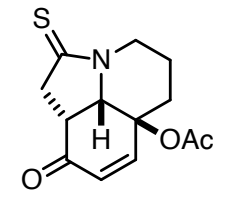

S8

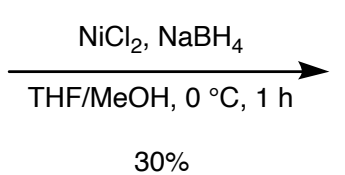

$30 \%$

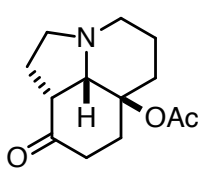

12

A solution of thiolactam $\mathbf{S} 8\left(41 \mathrm{mg}, 0.15 \mathrm{mmol}, 1.0\right.$ eq.) and $\mathrm{NiCl}_{2} \cdot 6 \mathrm{H}_{2} \mathrm{O}(293 \mathrm{mg}, 1.23 \mathrm{mmol}$, 8.0 eq.) in $\mathrm{THF} / \mathrm{MeOH}\left(1: 1,20 \mathrm{~mL}\right.$ ) was cooled to $0^{\circ} \mathrm{C} . \mathrm{NaBH}_{4}(134 \mathrm{mg}, 3.54 \mathrm{mmol}, 23$ eq.) was added portionwise over $5 \mathrm{~min}$ and the reaction mixture was stirred for $1 \mathrm{~h}$ at $0{ }^{\circ} \mathrm{C} . \mathrm{NaHCO}_{3}$ (sat. aq., $20 \mathrm{~mL})$ and $\mathrm{CH}_{2} \mathrm{Cl}_{2}(20 \mathrm{~mL})$ were added and the phases were separated. The aqueous phase extracted with $\mathrm{CH}_{2} \mathrm{Cl}_{2}(3 \times 20 \mathrm{~mL})$, the combined organic phases were dried over $\mathrm{MgSO}_{4}$, and the solvents were removed under reduced pressure. The crude product was pu-

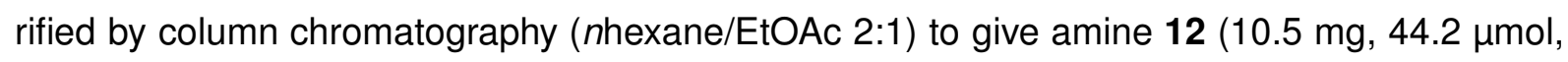
$30 \%)$ as a colorless oil.

TLC: $\mathrm{R}_{\mathrm{F}}=0.56$ (nhexane/EtOAc 1:1, CAM).

${ }^{1} \mathrm{H}-\mathrm{NMR}\left(600 \mathrm{MHz}, \mathrm{CDCl}_{3}\right): \delta=3.05(\mathrm{td}, J=9.0,3.0 \mathrm{~Hz}, 1 \mathrm{H}), 2.98(\mathrm{dt}, J=11.0,3.3 \mathrm{~Hz}, 1 \mathrm{H})$, 2.80 (ddd, $J=9.2,5.6,2.4 \mathrm{~Hz}, 1 \mathrm{H}), 2.74-2.71(\mathrm{~m}, 1 \mathrm{H}), 2.68-2.65(\mathrm{~m}, 1 \mathrm{H}), 2.49-2.40(\mathrm{~m}$, $3 \mathrm{H}), 2.39-2.37(\mathrm{~m}, 1 \mathrm{H}), 2.33-2.30(\mathrm{~m}, 1 \mathrm{H}), 2.08(\mathrm{~s}, 3 \mathrm{H}), 2.07-2.03(\mathrm{~m}, 1 \mathrm{H}), 1.95-1.90$ $(\mathrm{m}, 1 \mathrm{H}), 1.71(\mathrm{dtd}, J=12.8,9.8,3.0 \mathrm{~Hz}, 1 \mathrm{H}), 1.67-1.57(\mathrm{~m}, 3 \mathrm{H}) \mathrm{ppm}$.

${ }^{13} \mathrm{C}-\mathrm{NMR}\left(151 \mathrm{MHz}, \mathrm{CDCl}_{3}\right): \delta=210.4,170.4,81.871 .6,53.2,53.0,48.4,36.6,33.3,27.1$, 23.4, 22.6, $21.8 \mathrm{ppm}$.

IR (ATR): $\tilde{\nu}=2937,2797,1735,1715,1445,1368,1248,1150,1060,1019,769 \mathrm{~cm}^{-1}$.

HRMS (ESI-TOF): $m / z$ calculated for $\mathrm{C}_{13} \mathrm{H}_{20} \mathrm{NO}_{3}^{+}[\mathrm{M}+\mathrm{H}]^{+}:$238.1438; found 238.1442. 


\subsection{Synthesis of Hydrazine 13}

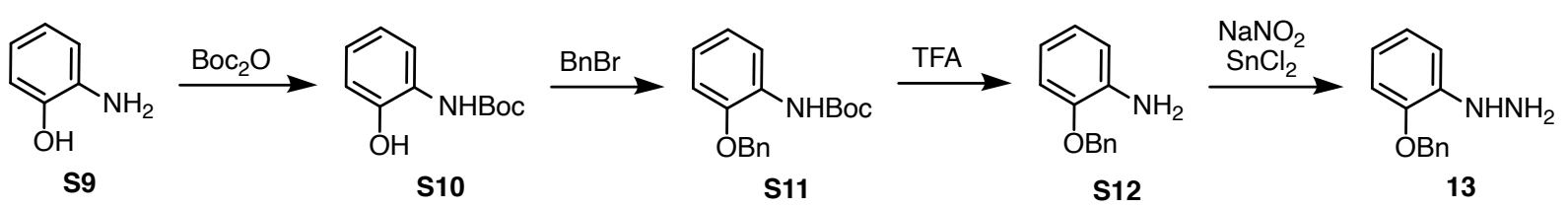

tert-Butyl (2-hydroxyphenyl)carbamate (S10) ${ }^{[4]}$

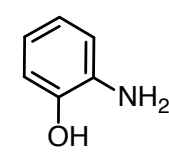

S9

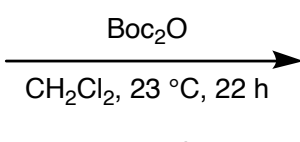

quant.<smiles>O=C(Nc1ccccc1)c1ccccc1</smiles>

S10

To a solution of 2-aminophenol (S9) $\left(5.00 \mathrm{~g}, 45.8 \mathrm{mmol}, 1.0\right.$ eq.) in $\mathrm{CH}_{2} \mathrm{Cl}_{2}(100 \mathrm{~mL})$ a solution of di-tert-butyl dicarbonate $\left(17.0 \mathrm{~mL}, 73.3 \mathrm{mmol}, 1.6\right.$ eq.) in $\mathrm{CH}_{2} \mathrm{Cl}_{2}(50 \mathrm{~mL})$ was added dropwise over $10 \mathrm{~min}$ at $23^{\circ} \mathrm{C}$. The solution was stirred for $22 \mathrm{~h}$ at $23^{\circ} \mathrm{C}$ and the solvent was removed under reduced pressure. The residue was triturated with ice-cold $n$ pentane $(3 \times 10 \mathrm{~mL})$ to give $\mathrm{N}$-protected 2-aminophenol $\mathbf{S 1 0}$ (9.58 g, $45.8 \mathrm{mmol}$, quant.) as colorless solid.

TLC: $\mathrm{R}_{\mathrm{F}}=0.29$ (nhexane/EtOAc 9:1, UV, CAM).

m.p.: $145-146^{\circ} \mathrm{C}$ (npentane/ $\mathrm{CH}_{2} \mathrm{Cl}_{2}$ ).

${ }^{1} \mathrm{H}-N M R\left(500 \mathrm{MHz}, \mathrm{CDCl}_{3}\right): \delta=8.12$ (bs, $\left.1 \mathrm{H}\right), 7.08$ (d, $\left.J=7.9 \mathrm{~Hz}, 1 \mathrm{H}\right), 7.04$ (ddd, $J=8.0,7.3$, $1.6 \mathrm{~Hz}, 1 \mathrm{H}$ ), 6.96 (dd, $J=8.1,1.5 \mathrm{~Hz}, 1 \mathrm{H}), 6.85$ (ddd, $J=7.9,7.3,1.5 \mathrm{~Hz}, 1 \mathrm{H}$ ), $6.66(\mathrm{bs}, 1 \mathrm{H}$ ), $1.53(\mathrm{~s}, 9 \mathrm{H}) \mathrm{ppm}$.

${ }^{13}$ C-NMR $\left(126 \mathrm{MHz}, \mathrm{CDCl}_{3}\right): \delta=155.2,147.7,125.8,125.7,121.6,120.9,119.1,82.2$, $28.4 \mathrm{ppm}$.

IR (ATR): $\tilde{\nu}=3328,2979,2934,1735,1688,1603,1523,1502,1454,1369,1229,1157,1054$, $748 \mathrm{~cm}^{-1}$.

All characterization data were consistent with those reported in the literature. ${ }^{[4]}$ 
tert-Butyl (2-(benzyloxy)phenyl)carbamate (S11) ${ }^{[4]}$<smiles>O=C(Nc1ccccc1)c1ccccc1</smiles>

S10

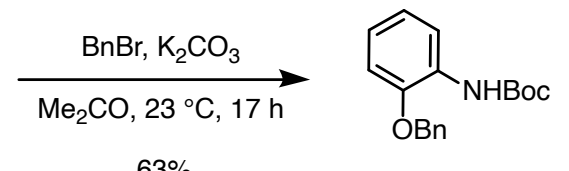

S11

$N$-Protected 2-aminophenol S10 (9.58 g, $45.8 \mathrm{mmol}, 1.0$ eq.) was dissolved in $\mathrm{Me}_{2} \mathrm{CO}(100 \mathrm{~mL})$. $\mathrm{K}_{2} \mathrm{CO}_{3}$ (9.50 g, $68.7 \mathrm{mmol}, 1.5$ eq.) and benzyl bromide (5.73 mL, $48.1 \mathrm{mmol}, 1.05$ eq.) were added at $23^{\circ} \mathrm{C}$ and the reaction was stirred for $17 \mathrm{~h}$ at this temperature. The reaction mixture was filtered and the filtrate was concentrated under reduced pressure. The residue was dissolved in EtOAc $(100 \mathrm{~mL})$, washed sequentially with $\mathrm{H}_{2} \mathrm{O}(2 \times 50 \mathrm{~mL})$ and brine (sat., $2 \times 50 \mathrm{~mL}$ ), and dried over $\mathrm{MgSO}_{4}$. The solvent was removed under reduced pressure and the crude product was purified by column chromatography (nhexane/EtOAc 25:1) to give $N$-Boc(2-benzyloxy)-aniline (S11) (7.54 g, $28.9 \mathrm{mmol}, 63 \%)$ as a colorless solid.

TLC: $\mathrm{R}_{\mathrm{F}}=0.64$ (nhexane/EtOAc 9:1, UV, CAM).

m.p.: $67-68^{\circ} \mathrm{C}(\mathrm{EtOAc})$.

${ }^{1} \mathrm{H}-N M R\left(500 \mathrm{MHz}, \mathrm{CDCl}_{3}\right): \delta=8.11(\mathrm{~d}, J=7.8 \mathrm{~Hz}, 1 \mathrm{H}), 7.43-7.39(\mathrm{~m}, 4 \mathrm{H}), 7.37-7.35(\mathrm{~m}$, $1 \mathrm{H}), 7.10$ (bs, $1 \mathrm{H}), 6.98-6.90(\mathrm{~m}, 3 \mathrm{H}), 5.12(\mathrm{~s}, 2 \mathrm{H}), 1.53(\mathrm{~s}, 9 \mathrm{H}) \mathrm{ppm}$.

${ }^{13} \mathrm{C}-\mathrm{NMR}\left(151 \mathrm{MHz}, \mathrm{CDCl}_{3}\right): \delta=152.9,146.9,136.7,128.9,128.6,128.4,127.7,122.4,121.6$, 118.5, 111.8, 80.5, 70.9, 28.5 ppm.

IR (ATR): $\tilde{\nu}=2977,1727,1602,1522,1446,1232,1152,1047,1022,741 \mathrm{~cm}^{-1}$.

All characterization data were consistent with those reported in the literature. ${ }^{[4]}$

\section{2-(Benzyloxy)aniline (S12) ${ }^{[4]}$}

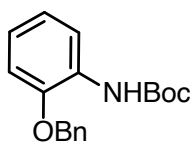

S11

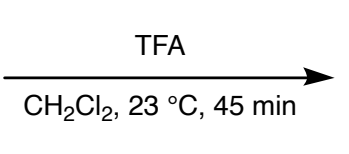

$83 \%$<smiles>Nc1ccccc1O</smiles>

$\mathrm{S} 12$

To a solution of $\mathrm{N}$-Boc-(2-benzyloxy)-aniline (S11) (1.35 g, $4.50 \mathrm{mmol}, 1.0$ eq.) in dry $\mathrm{CH}_{2} \mathrm{Cl}_{2}$ $(6 \mathrm{~mL})$ was added dropwise a solution of trifluoroacetic acid $(2.8 \mathrm{~mL})$ in $\operatorname{dry} \mathrm{CH}_{2} \mathrm{Cl}_{2}(2.8 \mathrm{~mL})$ at $23^{\circ} \mathrm{C}$. After the reaction mixture was stirred for $45 \mathrm{~min}$ at $23^{\circ} \mathrm{C}$, the solvent was removed 
under reduced pressure. $\mathrm{NaOH}(1 \mathrm{M}$ aq., $50 \mathrm{~mL}$ ) was added and the aqueous phase was extracted with $\mathrm{Et}_{2} \mathrm{O}(3 \times 25 \mathrm{~mL})$. The combined organic phases were dried over $\mathrm{MgSO}_{4}$ and the solvent was removed under reduced pressure. Purification by column chromatography (nhexane/EtOAc 25:1 $\rightarrow 4: 1$ ) gave aniline $\mathbf{S 1 2}$ (606 mg, $3.04 \mathrm{mmol}, 83 \%$ ) as a viscous oil.

TLC: $\mathrm{R}_{\mathrm{F}}=0.32$ (nhexane/EtOAc 9:1, UV, CAM).

${ }^{1} \mathrm{H}-N M R\left(500 \mathrm{MHz}, \mathrm{CDCl}_{3}\right): \delta=7.46-7.44(\mathrm{~m}, 2 \mathrm{H}), 7.41-7.38(\mathrm{~m}, 2 \mathrm{H}), 7.36-7.32(\mathrm{~m}, 1 \mathrm{H})$, $6.87(\mathrm{dd}, J=8.0,1.3 \mathrm{~Hz}, 1 \mathrm{H}), 6.82(\mathrm{td}, J=7.5,1.3 \mathrm{~Hz}, 1 \mathrm{H}), 6.76-6.70(\mathrm{~m}, 2 \mathrm{H}), 5.09(\mathrm{~s}, 2 \mathrm{H})$, 3.82 (bs, 2H) ppm.

${ }^{13} \mathrm{C}-\mathrm{NMR}\left(126 \mathrm{MHz}, \mathrm{CDCl}_{3}\right): \delta=146.6,137.4,136.7,128.7,128.1,127.7,121.7,118.5,115.4$, 112.3, $70.6 \mathrm{ppm}$.

IR (ATR): $\tilde{\nu}=3461,3371,3032,1613,1504,1454,1276,1211,1041,1013,738 \mathrm{~cm}^{-1}$. All characterization data were consistent with those reported in the literature. ${ }^{[4]}$

\section{(2-(Benzyloxy)phenyl)hydrazine (13)}

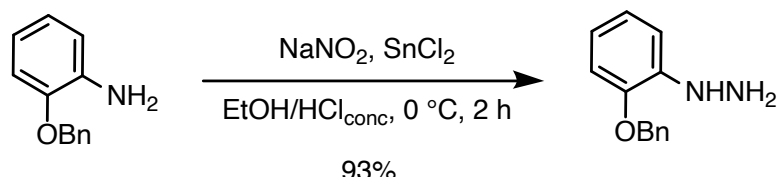

S12

To a suspension of anilin $\mathbf{S} 12$ (606 mg, $3.04 \mathrm{mmol}, 1.0$ eq.) in $\mathrm{EtOH}(10 \mathrm{~mL})$ and $\mathrm{HCl}(37 \%$ $w / w$ in $\mathrm{H}_{2} \mathrm{O}, 10 \mathrm{~mL}$ ), $\mathrm{NaNO}_{2}$ (220 mg, $3.19 \mathrm{mmol}, 1.05$ eq.) in $\mathrm{H}_{2} \mathrm{O}(2 \mathrm{~mL})$ was added dropwise at $0^{\circ} \mathrm{C}$ and the resulting mixture was stirred for $1 \mathrm{~h}$ at $0^{\circ} \mathrm{C}$. $\mathrm{SnCl}_{2} \cdot 2 \mathrm{H}_{2} \mathrm{O}(3.57 \mathrm{~g}, 15.8 \mathrm{mmol}$, 5.2 eq.) in $\mathrm{HCl}\left(37 \% w / w\right.$ in $\left.\mathrm{H}_{2} \mathrm{O}, 3 \mathrm{~mL}\right)$ was added dropwise and the mixture was stirred for $1 \mathrm{~h}$ at $0^{\circ} \mathrm{C}$. The reaction mixture was diluted with $\mathrm{H}_{2} \mathrm{O}(20 \mathrm{~mL})$ and the solution was basified with $\mathrm{NaOH}(\mathrm{pH}>9)$. The aqueous phase was extracted with $\mathrm{CH}_{2} \mathrm{Cl}_{2}(4 \times 30 \mathrm{~mL})$. The combined organic phases were dried over $\mathrm{MgSO}_{4}$ and the solvent was removed to give hydrazine 13 (616 $\mathrm{mg}, 2.84 \mathrm{mmol}, 93 \%)$ as a yellow oil that solidified upon refrigeration $\left(-20^{\circ} \mathrm{C}\right)$.

TLC: $\mathrm{R}_{\mathrm{F}}=0.10$ (nhexane/EtOAc 9:1, UV, CAM, $\mathrm{KMnO}_{4}$ ).

m.p.: $41-43^{\circ} \mathrm{C}\left(\mathrm{CH}_{2} \mathrm{Cl}_{2}\right)$.

${ }^{1} \mathrm{H}-N M R\left(500 \mathrm{MHz}, \mathrm{CDCl}_{3}\right): \delta=7.44-7.37(\mathrm{~m}, 4 \mathrm{H}), 7.36-7.32(\mathrm{~m}, 1 \mathrm{H}), 7.01(\mathrm{dd}, J=7.9$, $1.9 \mathrm{~Hz}, 1 \mathrm{H}), 6.98(\mathrm{dt}, J=7.1,1.4 \mathrm{~Hz}, 1 \mathrm{H}), 6.87(\mathrm{dd}, J=7.9,1.3 \mathrm{~Hz}, 1 \mathrm{H}), 6.77$ (ddd, $J=8.0$, 7.2, $1.9 \mathrm{~Hz}, 1 \mathrm{H}), 5.72(\mathrm{bs}, 1 \mathrm{H}), 5.07$ (s, 2H), 3.49 (bs, 2H) ppm. 
${ }^{1} \mathrm{H}-N M R\left(500 \mathrm{MHz},\left(\mathrm{D}_{3} \mathrm{C}\right)_{2} \mathrm{SO}\right): \delta=7.48(\mathrm{~d}, J=7.0 \mathrm{~Hz}, 2 \mathrm{H}), 7.39(\mathrm{t}, J=7.5 \mathrm{~Hz}, 2 \mathrm{H}), 7.32$ (t, $J=7.2 \mathrm{~Hz}, 1 \mathrm{H}), 7.04(\mathrm{dd}, J=7.8,1.6 \mathrm{~Hz}, 1 \mathrm{H}), 6.87(\mathrm{~d}, J=7.8 \mathrm{~Hz}, 1 \mathrm{H}), 6.82(\mathrm{t}, J=7.7 \mathrm{~Hz}$, $1 \mathrm{H}), 6.58(\mathrm{td}, J=7.7,1.6 \mathrm{~Hz}, 1 \mathrm{H}), 5.94(\mathrm{~s}, 1 \mathrm{H}), 5.08(\mathrm{~s}, 2 \mathrm{H}), 3.94(\mathrm{~s}, 2 \mathrm{H}) \mathrm{ppm}$.

${ }^{13} \mathrm{C}-\mathrm{NMR}\left(151 \mathrm{MHz}, \mathrm{CDCl}_{3}\right): \delta=145.8,141.4,137.0,128.7,128.2,127.7,121.8,118.7,111.4$, 110.9, 70.6 ppm.

${ }^{13}$ C-NMR $\left(126 \mathrm{MHz},\left(\mathrm{D}_{3} \mathrm{C}\right)_{2} \mathrm{SO}\right): 144.8,141.8,137.4,128.4,127.7,127.4,121.1,116.7,111.2$, $111.1,69.3 \mathrm{ppm}$.

IR (ATR): $\tilde{\nu}=3340,3061,3033,1597,1500,1437,1380,1256,1208,1013,738 \mathrm{~cm}^{-1}$.

HRMS (ESI-TOF): $m / z$ calculated for $\mathrm{C}_{13} \mathrm{H}_{14} \mathrm{~N}_{2} \mathrm{ONa}^{+}[\mathrm{M}+\mathrm{Na}]^{+}:$237.0998; found 237.0999.

The compound was described before, but has only been characterized by ${ }^{1} \mathrm{H}-\mathrm{NMR}$ in $\left(\mathrm{D}_{3} \mathrm{C}\right)_{2} \mathrm{SO} .{ }^{[5]}$

\subsection{Fragment Coupling}
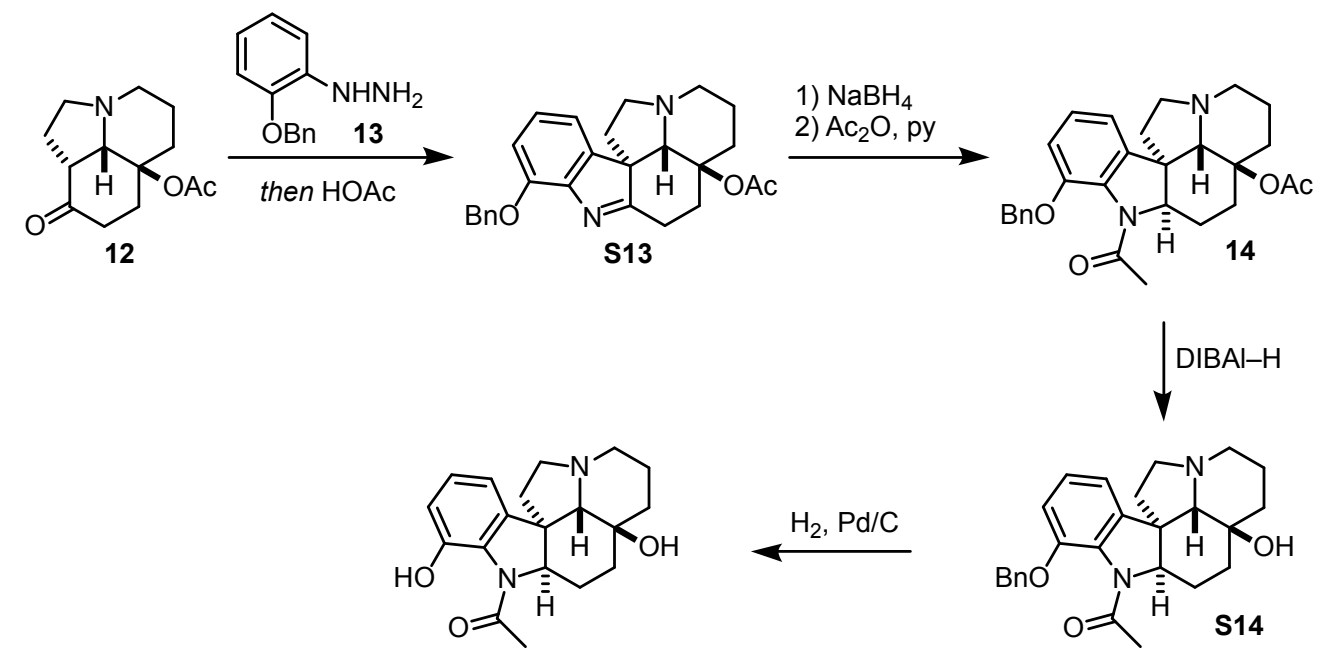

aspidodispermine (1)

\section{O-Acetyl-170-benzyl aspidodispermine (14)}

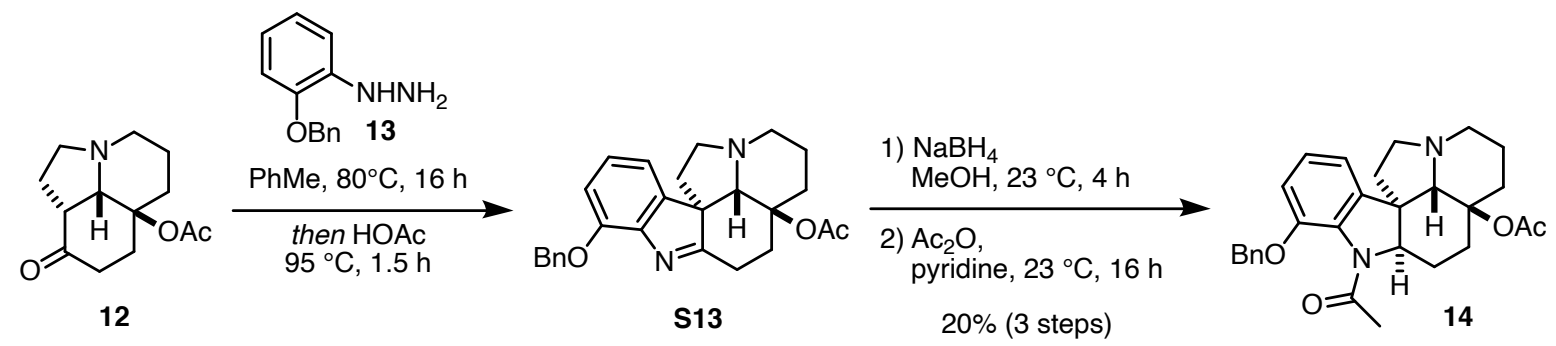

A mixutre of amine 12 (20.5 mg, $86.8 \mu \mathrm{mol}, 1.0$ eq.), hydrazine 13 (27.9 mmol, $130 \mu \mathrm{mol}$, 1.5 eq.), and $3 \AA$ molecular sieves $(20 \mathrm{mg})$ in dry toluene $(1 \mathrm{~mL})$ was stirred for $18 \mathrm{~h}$ at 
$80^{\circ} \mathrm{C}$. The reaction mixture was cooled to $23^{\circ} \mathrm{C}$ and filtered. The solvent of the filtrate was removed under reduced pressure and the residue was dissolved in $\mathrm{HOAc}(1 \mathrm{~mL})$. After stirring the reaction mixture for $90 \mathrm{~min}$ at $95^{\circ} \mathrm{C}, \mathrm{Na}_{2} \mathrm{CO}_{3}$ (sat. aq., $15 \mathrm{~mL}$ ) was added $(\mathrm{pH}>9)$. The aqueous phase was extracted with $\mathrm{CH}_{2} \mathrm{Cl}_{2}(3 \times 10 \mathrm{~mL})$. The combined organic phases were dried over $\mathrm{MgSO}_{4}$ and the solvent was removed under reduced pressure. The crude product was purified by column chromatopgraphy (nhexane/EtOAc 1:1) to yield indolenin $\mathbf{S 1 3}(12 \mathrm{mg})$ as a yellow oil which still contained impurities.

To indolenin $\mathbf{S} 13(12 \mathrm{mg})$ in $\mathrm{MeOH}(1 \mathrm{~mL})$ was added $\mathrm{NaBH}_{4}(5.4 \mathrm{mg})$ at $23^{\circ} \mathrm{C}$ and the resulting mixture was stirred for $4 \mathrm{~h}$ at this temperature. $\mathrm{NaHCO}_{3}$ (sat. aq., $10 \mathrm{~mL}$ ) was added and the aqueous phase was extracted with $\mathrm{CH}_{2} \mathrm{Cl}_{2}(4 \times 10 \mathrm{~mL})$. The combined organic phases were dried over $\mathrm{MgSO}_{4}$ and the solvent was removed under reduced pressure. The crude product was purified by column chromatography (silica gel, $\mathrm{CH}_{2} \mathrm{Cl}_{2} / \mathrm{MeOH} 50: 1$ ) to yield the indole $(9 \mathrm{mg})$.

$\mathrm{Ac}_{2} \mathrm{O}(0.2 \mathrm{~mL})$ was added to a solution of the indole $(9 \mathrm{mg})$ in pyridine $(0.6 \mathrm{~mL})$ and the resulting mixture was stirred for $16 \mathrm{~h}$ at $23^{\circ} \mathrm{C} . \mathrm{NH}_{3}(30 \% \mathrm{w} / \mathrm{w}$ aq., $10 \mathrm{~mL})$ was added and the aqueous phase was extracted with $\mathrm{CH}_{2} \mathrm{Cl}_{2}(4 \times 10 \mathrm{~mL})$. The combined organic phases were dried over $\mathrm{MgSO}_{4}$ and the solvent was removed under reduced pressure. The crude product was purified by column chromatography $\left(\mathrm{CH}_{2} \mathrm{Cl}_{2} / \mathrm{MeOH}, 50: 1\right)$ to yield protected aspidodispermine 14 (8.1 mg, $17 \mu \mathrm{mol}, 20 \%$ over 3 steps) as colorless oil.

TLC: $\mathrm{R}_{\mathrm{F}}=0.37\left(\mathrm{CH}_{2} \mathrm{Cl}_{2} / \mathrm{MeOH} 19: 1, \mathrm{UV}, \mathrm{CAM}\right)$.

${ }^{1} \mathrm{H}-\mathrm{NMR}\left(600 \mathrm{MHz}, \mathrm{CD}_{3} \mathrm{CN}, 65^{\circ} \mathrm{C}\right): \delta=7.41(\mathrm{~d}, J=7.4 \mathrm{~Hz}, 2 \mathrm{H}), 7.36(\mathrm{t}, J=7.3 \mathrm{~Hz}, 2 \mathrm{H}), 7.32$ $-7.30(\mathrm{~m}, 1 \mathrm{H}), 7.09-7.06(\mathrm{~m}, 1 \mathrm{H}), 6.94(\mathrm{~d}, J=7.6 \mathrm{~Hz}, 2 \mathrm{H}), 5.16-5.14(\mathrm{~m}, 2 \mathrm{H}), 4.43-4.40$ (m, 1H), $3.17(\mathrm{td}, J=9.0,3.6 \mathrm{~Hz}, 1 \mathrm{H}), 3.00(\mathrm{~d}, J=11.2 \mathrm{~Hz}, 1 \mathrm{H}), 2.82(\mathrm{~s}, 1 \mathrm{H}), 2.40-2.36(\mathrm{~m}$, $2 \mathrm{H}$ ), 2.22 (td, $J=14.1,3.7 \mathrm{~Hz}, 1 \mathrm{H}$ ), $2.15(\mathrm{~s}, 3 \mathrm{H}), 2.12-2.03(\mathrm{~m}, 4 \mathrm{H}), 1.82$ (ddd, $J=17.1,6.5$, $3.5 \mathrm{~Hz}, 1 \mathrm{H}), 1.77-1.73(\mathrm{~m}, 1 \mathrm{H}), 1.67-1.62(\mathrm{~m}, 3 \mathrm{H}), 1.36(\mathrm{~s}, 3 \mathrm{H}) \mathrm{ppm}$.

${ }^{13} \mathrm{C}-N M R\left(151 \mathrm{MHz}, \mathrm{CD}_{3} \mathrm{CN}, 65^{\circ} \mathrm{C}\right): \delta=170.6(2 \mathrm{C}), 150.2,143.9,138.6,131.8,129.7,129.1$, $129.1,127.0,117.8,115.4,82.7,72.3,69.7,69.2,54.6,53.9,53.1,38.4,34.8,25.6,25.6,24.3$, 23.5, $22.0 \mathrm{ppm}$.

IR (ATR): $\tilde{\nu}=2932,2864,2791,1733,1653,1485,1455,1377,1249,1210,1115,1026$, $773 \mathrm{~cm}^{-1}$.

HRMS (ESI-TOF): $m / z$ calculated for $\mathrm{C}_{28} \mathrm{H}_{33} \mathrm{~N}_{2} \mathrm{O}_{4}^{+}[\mathrm{M}+\mathrm{H}]^{+}:$461.2435; found 461.2446. 

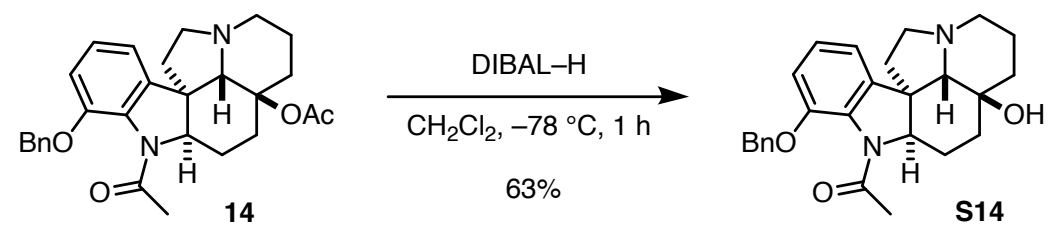

A solution of protected aspidodispermine 14 (4.0 mg, $8.7 \mu \mathrm{mol}, 1.0$ eq.) in dry $\mathrm{CH}_{2} \mathrm{Cl}_{2}$ was cooled to $-78^{\circ} \mathrm{C}$. DIBAL-H ( $1 \mathrm{M}$ in hexane, $10 \mu \mathrm{L}, 10 \mu \mathrm{mol}, 1.15$ eq.) was added and the reaction mixture was stirred for $1 \mathrm{~h}$ at $-78^{\circ} \mathrm{C} . \mathrm{H}_{2} \mathrm{O}(5 \mu \mathrm{L})$ was added and the reaction mixture was warmed to $23^{\circ} \mathrm{C}$. $\mathrm{NaOH}(15 \% w / w$ aq., $5 \mu \mathrm{L})$ and $\mathrm{H}_{2} \mathrm{O}(15 \mu \mathrm{L})$ were added and the mixture was stirred for $15 \mathrm{~min}$ before $\mathrm{MgSO}_{4}$ was added and stirring was continued for $30 \mathrm{~min}$. The mixture was filtered through a plug of Celite ${ }^{\circledR}$ and the solvent was removed under reduced pressure. The crude product was purified by column chromatography $\left(\mathrm{CH}_{2} \mathrm{Cl}_{2} / \mathrm{MeOH} 50: 1 \rightarrow 19: 1\right)$ to give alcohol S14 (2.3 mg, $5.5 \mu \mathrm{mol}, 63 \%)$ as a colorless oil.

TLC: $\mathrm{R}_{\mathrm{F}}=0.30\left(\mathrm{CH}_{2} \mathrm{Cl}_{2} / \mathrm{MeOH} 19: 1, \mathrm{UV}, \mathrm{CAM}\right)$.

${ }^{1} \mathrm{H}-N M R\left(700 \mathrm{MHz}, \mathrm{CDCl}_{3}\right): \delta=7.42-7.49(\mathrm{~m}, 2 \mathrm{H}), 7.37(\mathrm{t}, J=7.6 \mathrm{~Hz}, 2 \mathrm{H}), 7.33-7.30(\mathrm{~m}$, $1 \mathrm{H}), 7.09(\mathrm{dd}, J=8.2,7.4 \mathrm{~Hz}, 1 \mathrm{H}), 6.95(\mathrm{~d}, J=7.4 \mathrm{~Hz}, 1 \mathrm{H}), 6.90(\mathrm{~d}, J=8.7 \mathrm{~Hz}, 1 \mathrm{H}), 5.13-$ $5.09(\mathrm{~m}, 2 \mathrm{H}), 4.58$ (bs, $1 \mathrm{H}), 3.17(\mathrm{td}, J=9.1,3.3 \mathrm{~Hz}, 1 \mathrm{H}), 3.01(\mathrm{~d}, J=11.3 \mathrm{~Hz}, 1 \mathrm{H}), 2.42$ (s, $1 \mathrm{H}$ ), $2.34(\mathrm{q}, J=9.0 \mathrm{~Hz}, 1 \mathrm{H}$ ), $2.24-2.17(\mathrm{~m}, 4 \mathrm{H}), 2.10(\mathrm{dt}, J=13.3,8.7 \mathrm{~Hz}, 1 \mathrm{H}$ ), 2.02 (td, $J=11.3,3.5 \mathrm{~Hz}, 1 \mathrm{H}), 1.97-1.92(\mathrm{~m}, 1 \mathrm{H}), 1.72(\mathrm{~d}, J=14.3 \mathrm{~Hz}, 1 \mathrm{H}), 1.69-1.61(\mathrm{~m}, 5 \mathrm{H}), 1.42$ (td, $J=12.8,5.2 \mathrm{~Hz}, 1 \mathrm{H}), 1.19(\mathrm{~d}, J=14.0 \mathrm{~Hz}, 1 \mathrm{H}) \mathrm{ppm}$.

${ }^{13} \mathrm{C}-\mathrm{NMR}\left(176 \mathrm{MHz}, \mathrm{CDCl}_{3}\right): \delta=173.3,149.3,142.7,136.6,130.3,128.8,128.3,127.7,126.6$, 115.8, 113.9, 71.3, 71.0, 70.5, 68.8, 53.1, 52.4, 38.6, 37.3, 29.8, 28.7, 24.0, 23.55, 23.52 ppm. IR (ATR): $\tilde{\nu}=3420,2930,2856,2785,1623,1488,1457,1387,1271,1197,1055,942$, $755 \mathrm{~cm}^{-1}$.

HRMS (ESI-TOF): $m / z$ calculated for $\mathrm{C}_{26} \mathrm{H}_{31} \mathrm{~N}_{2} \mathrm{O}_{3}^{+}[\mathrm{M}+\mathrm{H}]^{+}:$419.2329; found 419.2342. 

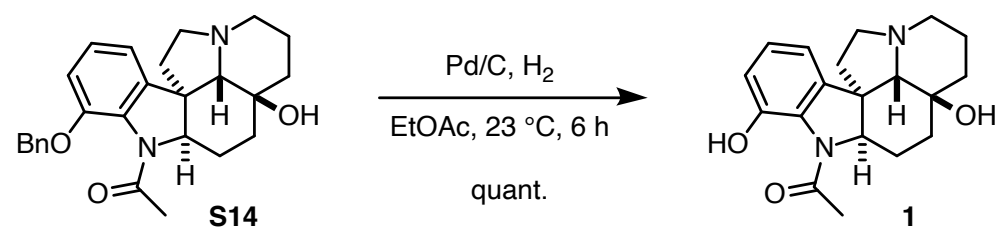

$\mathrm{Pd} / \mathrm{C}(10 \% \mathrm{w} / \mathrm{w}, 2.4 \mathrm{mg}, 2.3 \mu \mathrm{mol}, 0.3$ eq.) was added to a solution of 170-benzyl aspidodispermine (S14) $(3.0 \mathrm{mg}, 7.2 \mu \mathrm{mol}, 1.0$ eq.) in EtOAc $(1 \mathrm{~mL})$ and the resulting mixture was stirred under an atmosphere of hydrogen (balloon) for $6 \mathrm{~h}$ at $23^{\circ} \mathrm{C}$. The reaction mixture was filtered through a plug of Celite ${ }^{\circledR}$ and the solvent was removed under reduced pressure to give aspidodispermine (1) (2.3 mg, $7.2 \mu \mathrm{mol}$, quant.) as a colorless oil.

TLC: $\mathrm{R}_{\mathrm{F}}=0.35\left(\mathrm{CH}_{2} \mathrm{Cl}_{2} / \mathrm{MeOH} 19: 1\right.$, UV, CAM).

${ }^{1} \mathrm{H}-N M R\left(700 \mathrm{MHz}, \mathrm{CDCl}_{3}\right): \delta=10.81(\mathrm{~s}, 1 \mathrm{H}), 7.09(\mathrm{t}, J=7.7 \mathrm{~Hz}, 1 \mathrm{H}), 6.84(\mathrm{dd}, J=8.2,1.2 \mathrm{~Hz}$, $1 \mathrm{H}), 6.79(\mathrm{dd}, J=7.4,1.2 \mathrm{~Hz}, 1 \mathrm{H}), 4.06(\mathrm{dd}, J=11.6,5.9 \mathrm{~Hz}, 1 \mathrm{H}), 3.19(\mathrm{td}, J=9.1,3.2 \mathrm{~Hz}$, 1H), $3.04(\mathrm{~d}, J=11.3 \mathrm{~Hz}, 1 \mathrm{H}), 2.47(\mathrm{~s}, 1 \mathrm{H}), 2.37$ (dd, $J=18.2,9.3 \mathrm{~Hz}, 1 \mathrm{H}), 2.33(\mathrm{~s}, 3 \mathrm{H}), 2.20$ (td, $J=14.1,3.7 \mathrm{~Hz}, 1 \mathrm{H}), 2.12-2.07(\mathrm{~m}, 1 \mathrm{H}), 2.07-2.03(\mathrm{~m}, 1 \mathrm{H}), 1.89$ (ddt, $J=13.3,6.4$, $3.5 \mathrm{~Hz}, 1 \mathrm{H}), 1.84-1.75(\mathrm{~m}, 2 \mathrm{H}), 1.70-1.64(\mathrm{~m}, 3 \mathrm{H}), 1.48(\mathrm{td}, J=12.7,6.2 \mathrm{~Hz}, 1 \mathrm{H}), 1.40$ (s, $1 \mathrm{H}), 1.31-1.28(\mathrm{~m}, 1 \mathrm{H}) \mathrm{ppm}$.

${ }^{13} \mathrm{C}-\mathrm{NMR}\left(176 \mathrm{MHz}, \mathrm{CDCl}_{3}\right): \delta=169.3,147.7,139.9,128.3,127.1,118.4,113.2,70.7,70.1$, 69.1, 53.2, 52.6, 52.5, $38.4(2 \mathrm{C}), 28.6,24.4,23.5,22.9 \mathrm{ppm}$.

IR (ATR): $\tilde{\nu}=3404,2930,2855,2791,1630,1601,1575,1473,1448,1391,1262,956,935$, $784,735 \mathrm{~cm}^{-1}$.

HRMS (ESI-TOF): $m / z$ calculated for $\mathrm{C}_{19} \mathrm{H}_{25} \mathrm{~N}_{2} \mathrm{O}_{3}[\mathrm{M}+\mathrm{H}]^{+}:$219.1860; found 219.1865.

Table S 2: ${ }^{1} \mathrm{H}-\mathrm{NMR}$ comparison for 1.

Shifts are given in ppm. Parameters (e.g. MHz, solvent) for aquisition of ${ }^{1} \mathrm{H}-\mathrm{NMR}$ of natural sample are unknown. ${ }^{[6]}$ Only the three values indicated below were reported in reference [6]. For synthetic $10.03 \mathrm{ppm}$ were substracted from the measured values.

\begin{tabular}{c|c|c}
$\#$ & Natural 1 & Synthetic 1 \\
\hline \hline 2 & 4.03 & 4.03 \\
$17-\mathrm{OH}$ & 10.82 & 10.78 \\
21 & 2.30 & 2.30 \\
\hline
\end{tabular}


Table S 3: ${ }^{1} \mathrm{H}$ - and ${ }^{13} \mathrm{C}-\mathrm{NMR}$ assignment for 1 .

All chemical shifts are reported in ppm. Coupling constants are in parentheses and are reported in $\mathrm{Hz}$. Spectra were measured in $\mathrm{CDCl}_{3}$ and are referenced to the residual sovent peak $\left({ }^{1} \mathrm{H} \delta=7.26 \mathrm{ppm},{ }^{13} \mathrm{C} \delta=77.16 \mathrm{ppm}\right)$.

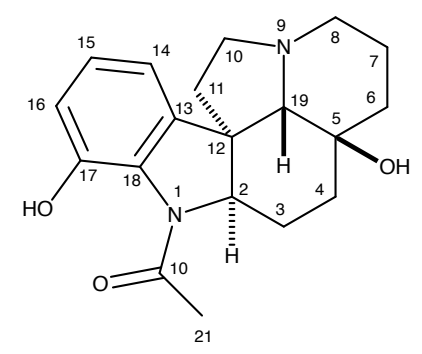

\begin{tabular}{|c|c|c|}
\hline \# & ${ }^{1} \mathrm{H}$ & ${ }^{13} \mathrm{C}$ \\
\hline 1 & - & - \\
\hline 2 & 4.06 & 69.1 \\
\hline 3 & $1.89 \mathrm{ddt}(13.3,6.4,3.5), 1.84-1.75 \mathrm{~m}$ & 24.4 \\
\hline 4 & $2.20 \mathrm{dt}(14.1,3.7), 1.31-1.28 \mathrm{~m}$ & 28.6 \\
\hline 5 & - & 70.1 \\
\hline $5-\mathrm{OH}$ & $1.40 \mathrm{~s}$ & - \\
\hline 6 & $1.84-1.75 \mathrm{~m}, 1.48 \mathrm{td}(12.7,6.2)$ & 38.4 \\
\hline 7 & $1.70-1.64 \mathrm{~m}$ & 23.5 \\
\hline 8 & $3.04 \mathrm{~d}(11.3), 2.07-2.03 \mathrm{~m}$ & 53.2 \\
\hline 9 & - & - \\
\hline 10 & $3.19 \mathrm{td}(9.1,3.2), 2.37 \mathrm{dd}(18.2,9.3)$ & 52.5 \\
\hline 11 & $2.12-2.07 \mathrm{~m}, 1.70-1.64 \mathrm{~m}$ & 38.4 \\
\hline 12 & - & 52.6 \\
\hline 13 & - & 139.9 \\
\hline 14 & $6.79 \mathrm{dd}(7.4,1.2)$ & 113.2 \\
\hline 15 & $7.09 \mathrm{t}(7.7)$ & 128.4 \\
\hline 16 & $6.84 \mathrm{dd}(8.2,1.2)$ & 118.4 \\
\hline 17 & - & 147.7 \\
\hline $17-\mathrm{OH}$ & $10.81 \mathrm{~s}$ & - \\
\hline 18 & - & 127.1 \\
\hline 19 & $2.47 \mathrm{~s}$ & 70.7 \\
\hline 20 & - & 169.3 \\
\hline 21 & $2.33 \mathrm{~s}$ & 22.9 \\
\hline
\end{tabular}




\section{References}

[1] A. Berkessel, S. Das, D. Pekel and J. M. Neudörfl, Anion-Binding Catalysis by ElectronDeficient Pyridinium Cations, Angew. Chem. Int. Ed., 2014, 53, 11660-11664.

[2] S. Olguín-Uribe, M. V. Mijangos, Y. A. Amador-Sánchez, M. A. Sánchez-Carmona and L. D. Miranda, Expedited Synthesis of Matrine Analogues through an Oxidative Cascade Addition/Double-Cyclization Radical Process, Eur. J. Org. Chem., 2017, 2481-2485.

[3] C. Frauenfelder, H.-J. Borschberg, Towards a New Synthetic Entry into the Iboga-Alkaloid Family, Helv. Chim. Acta 2000, 83, 1753-1765.

[4] N. P. Dubasch, N. K. Mangu, A. Satyam, Synthesis of 7-Alkoxy/Hydroxy- $\alpha$-methyltryptamines, Synth. Commun. 2004, 34, 1791-1799.

[5] A. Dossetter, N. Heron, 1-Cyanocyclopropyl-Derivatives as Cathepsin K Inhibitors, WO 2009/001129 A1, 2008.

[6] M. Ikeda, C. Djerassi, Alkaloid Studies LX. The Structures of Two Novel Aspidosperma Alkaloids, Deoxyaspidodispermine and Aspidodispermine, Tetrahedron Lett. 1968, 9, 5837-5840. 


\section{NMR Spectra}

\subsection{New Compounds}
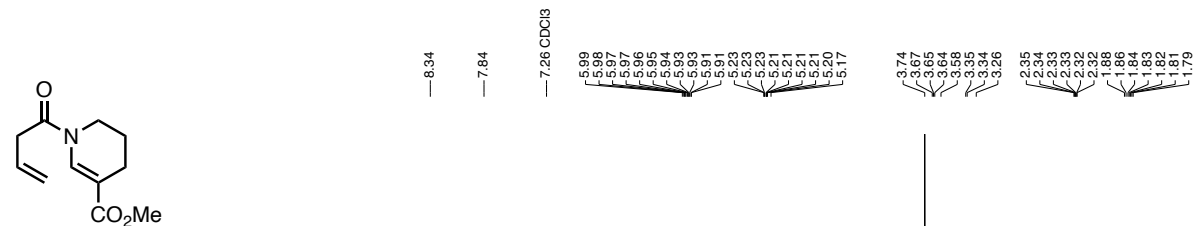

9, $500 \mathrm{MHz}, \mathrm{CDCl}_{3}$
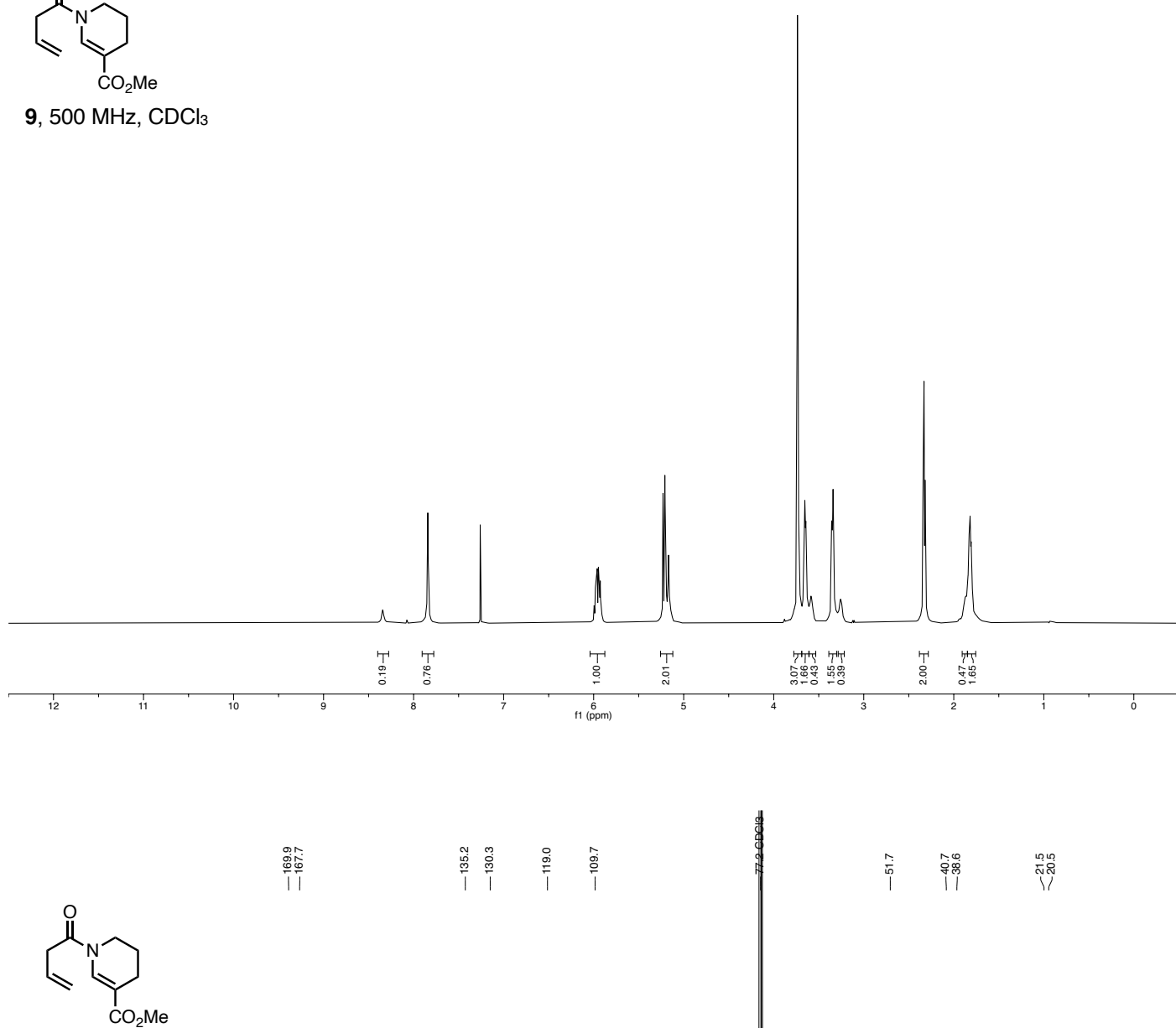

9, $126 \mathrm{MHz}, \mathrm{CDCl}_{3}$

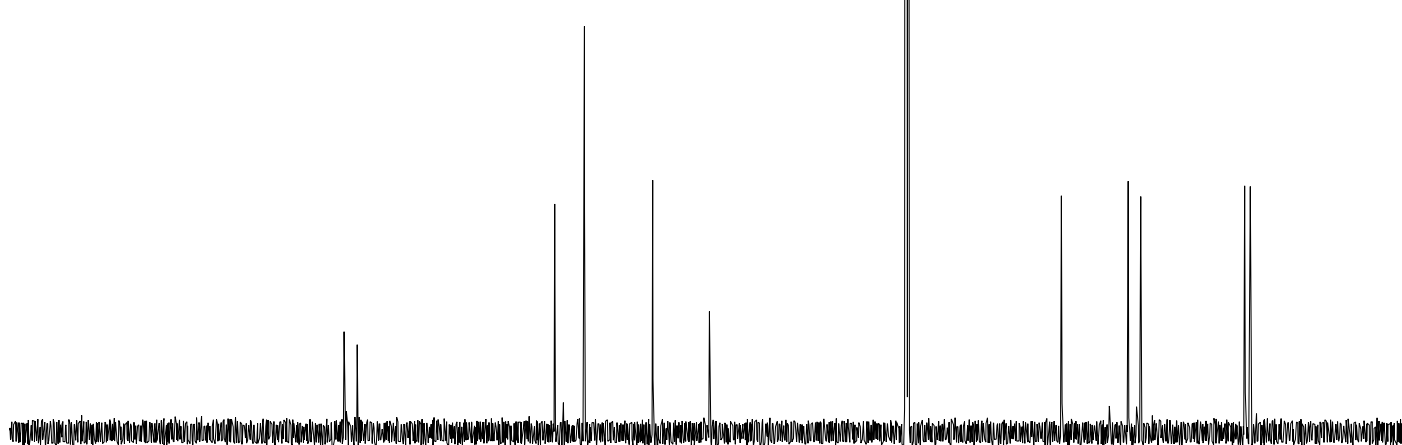




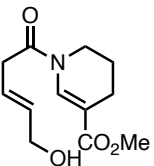

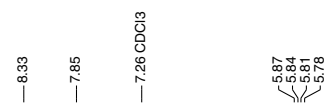

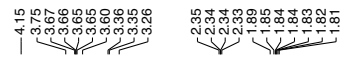

8, $500 \mathrm{MHz} \mathrm{CDCl}_{3}$
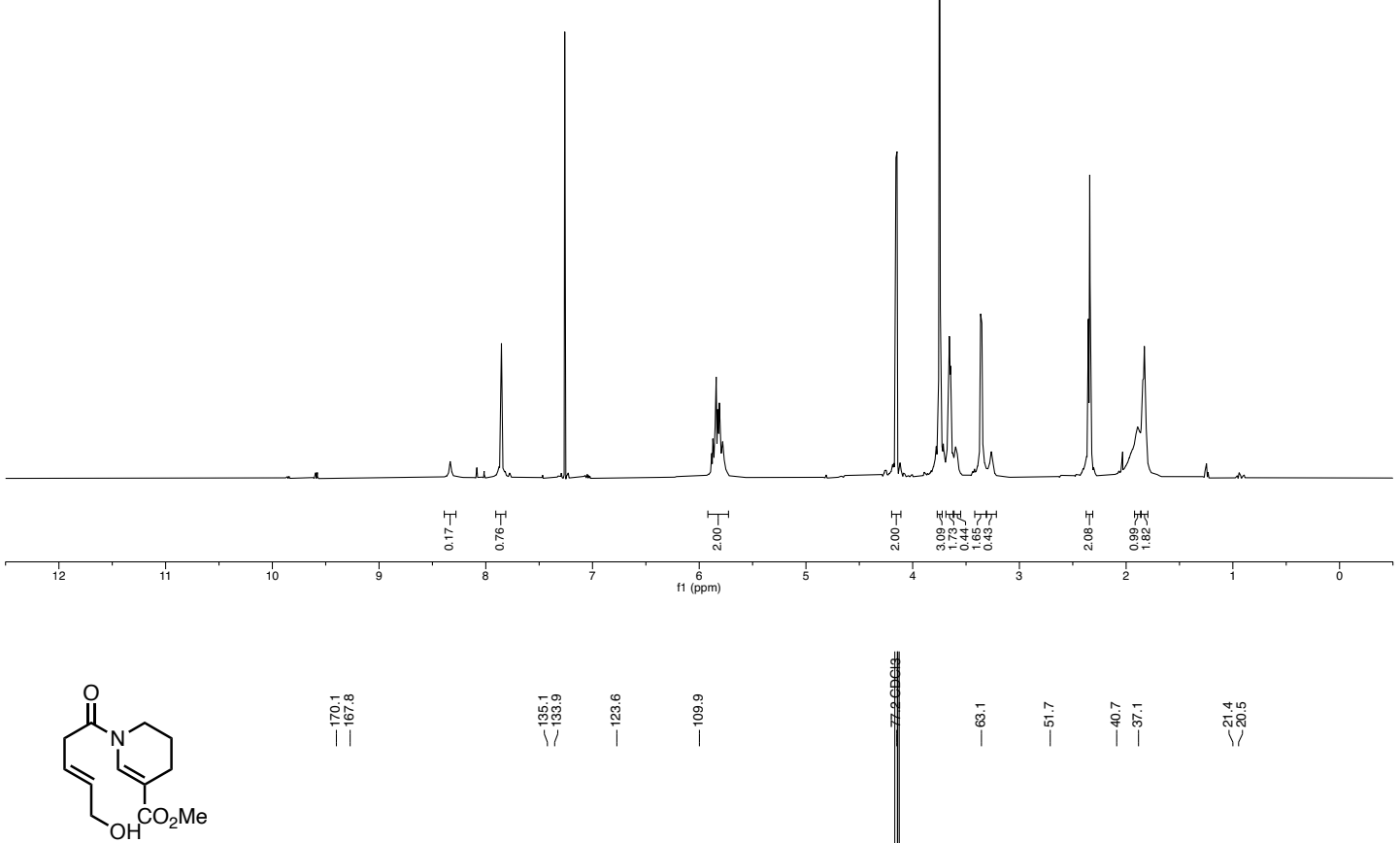

8, $126 \mathrm{MHz}, \mathrm{CDCl}_{3}$
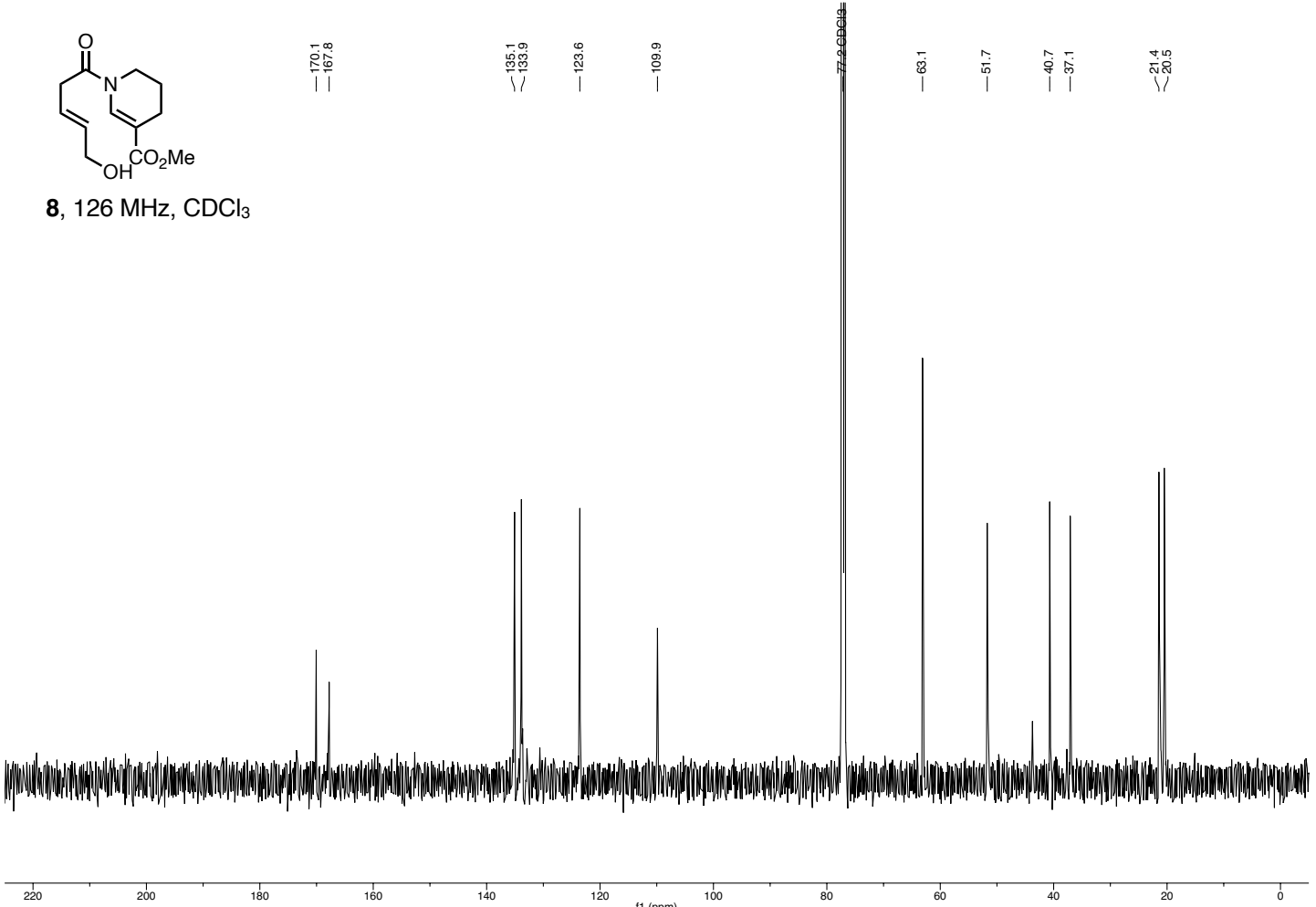


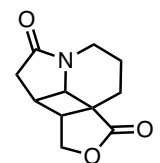

10, $500 \mathrm{MHz}, \mathrm{CDCl}_{3}$
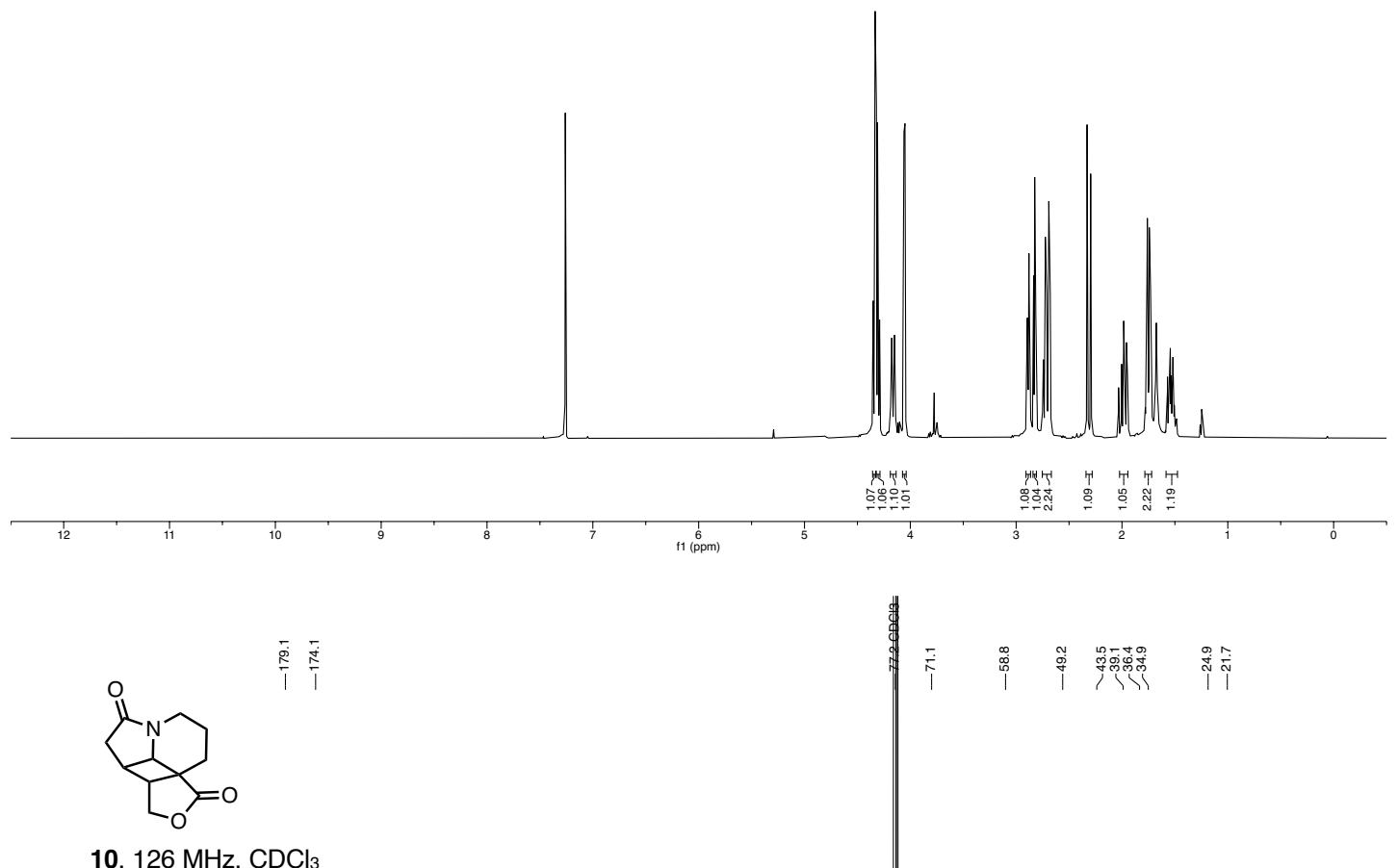

10, $126 \mathrm{MHz}^{\mathrm{CDCl}_{3}}$
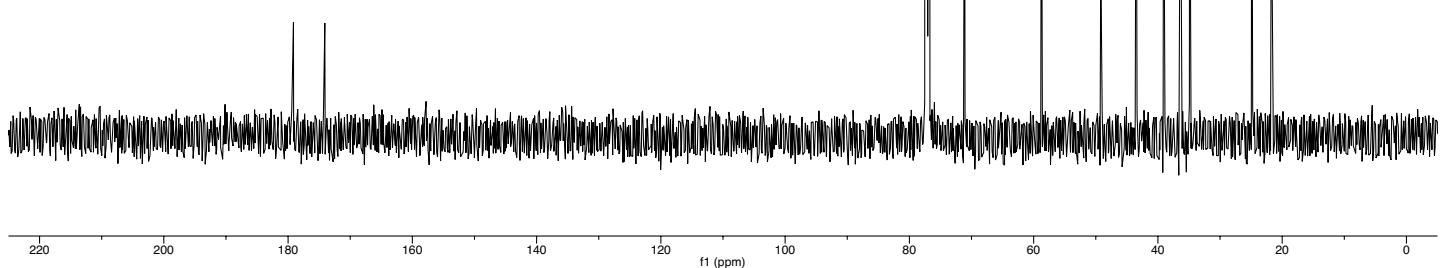


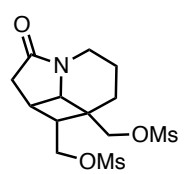

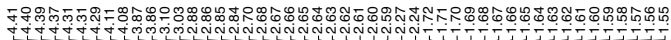

S5, $500 \mathrm{MHz}, \mathrm{CDCl}_{3}$
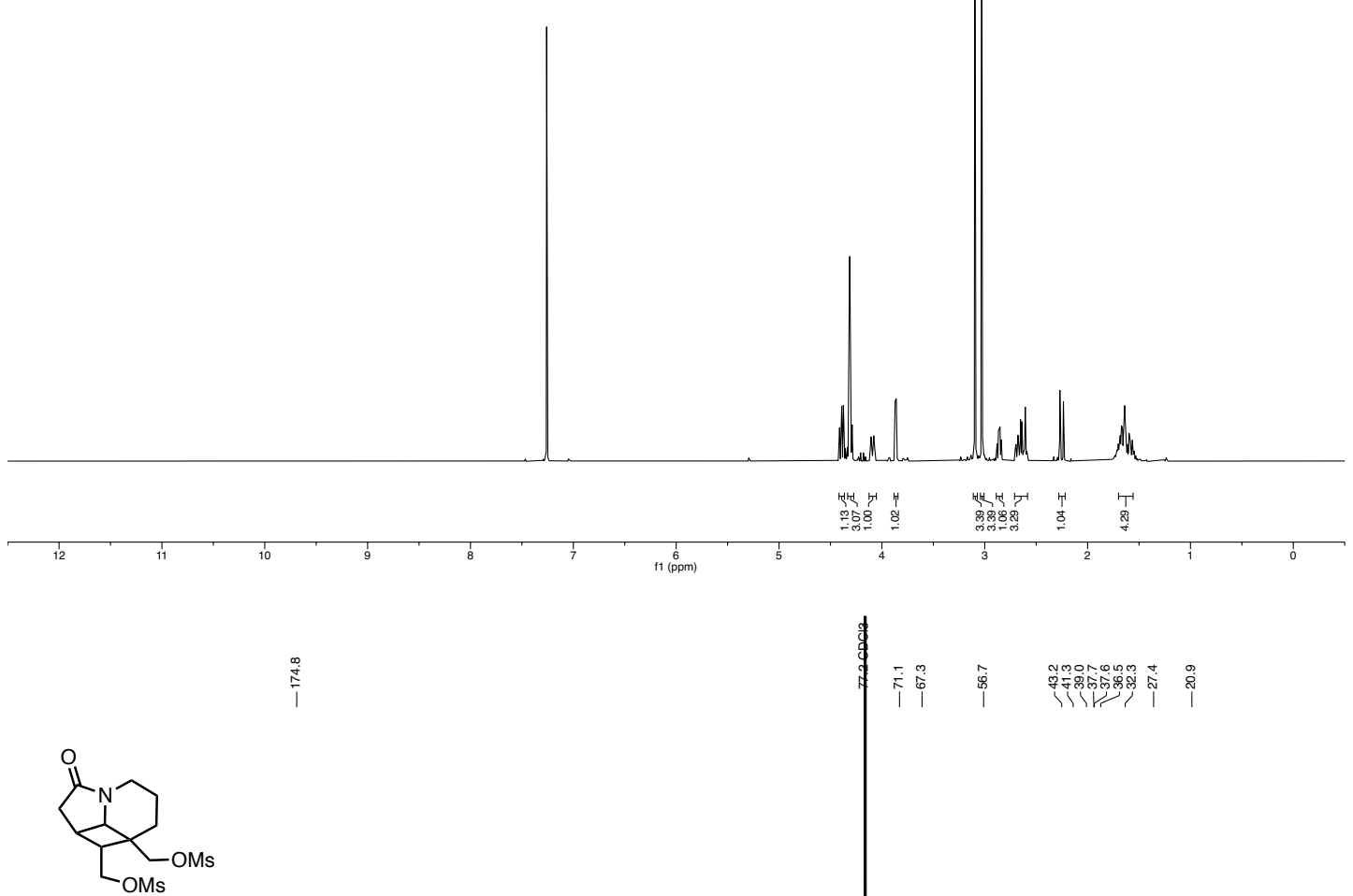

S5, $151 \mathrm{MHz}, \mathrm{CDCl}_{3}$
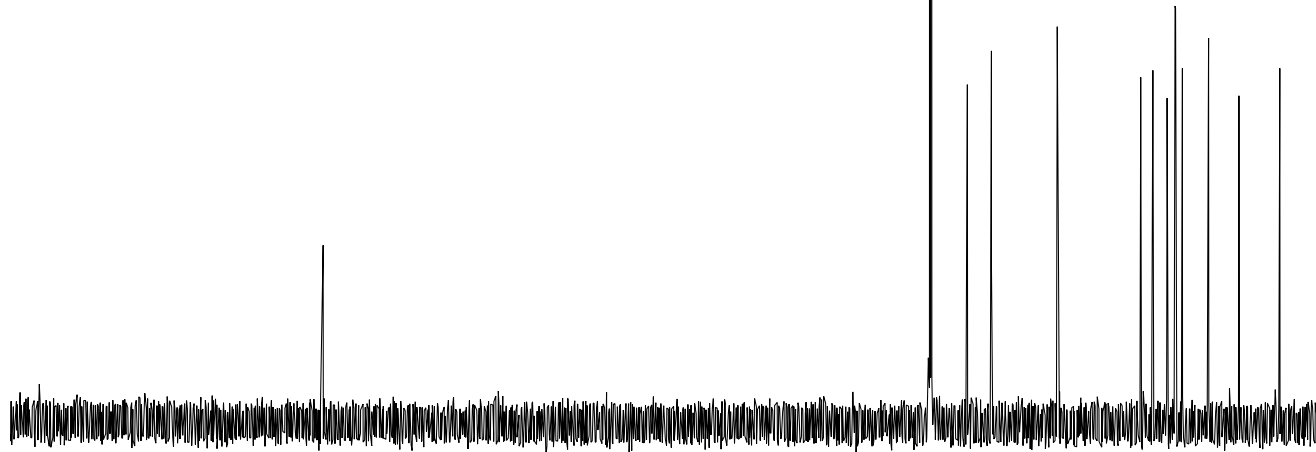

220

$120 \quad \mathrm{H}(\mathrm{ppm})$ 
<smiles>O=C1CC2C3CSCC34CCCN1C24</smiles>

S6, $500 \mathrm{MHz}, \mathrm{CDCl}_{3}$

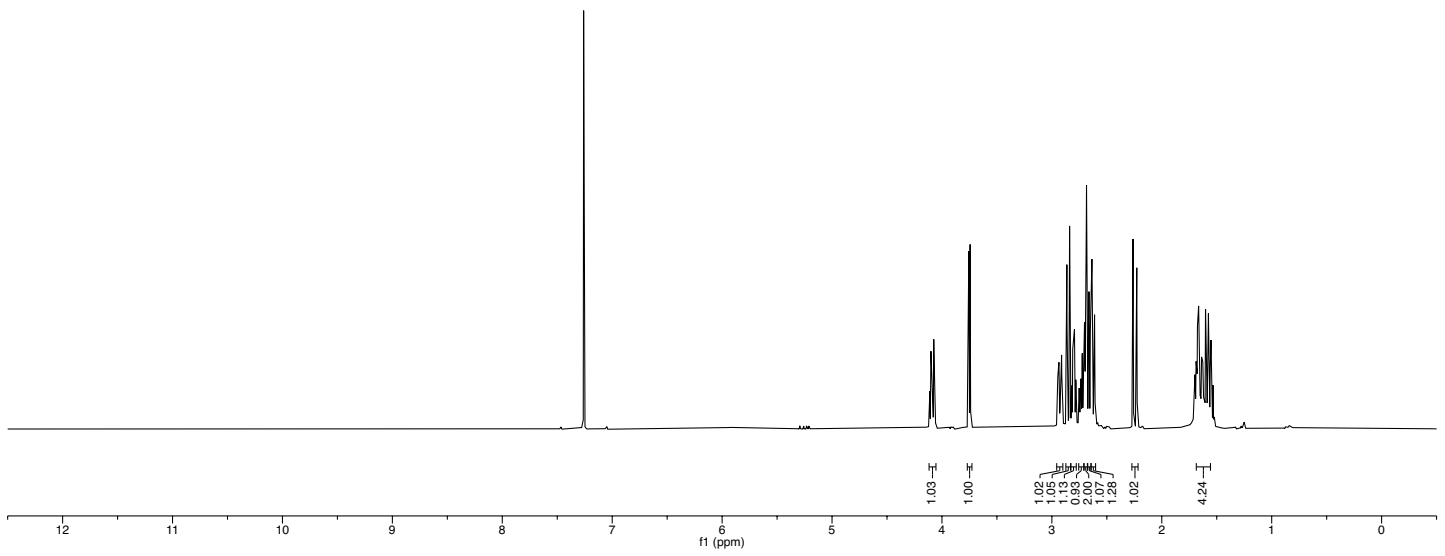<smiles>O=C1CC2C3CSCC34CCCN1C24</smiles>

S6, $126 \mathrm{MHz}, \mathrm{CDCl}_{3}$
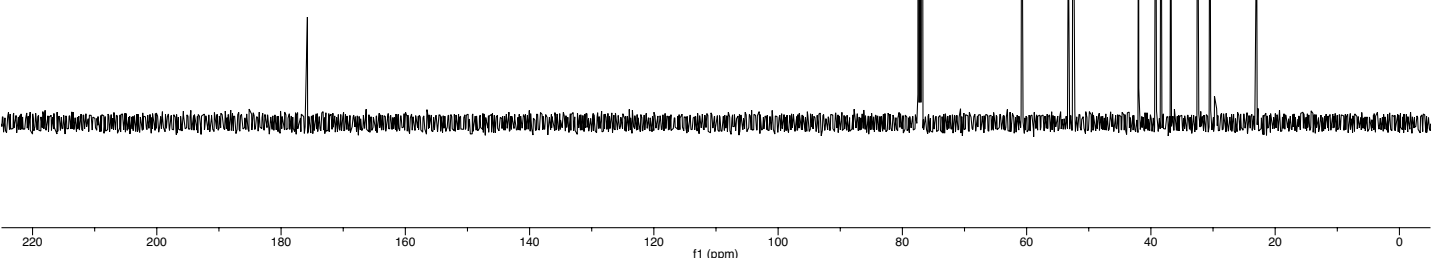
魚

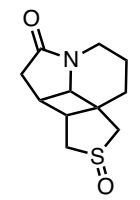

7, $500 \mathrm{MHz}, \mathrm{CDCl}_{3}$
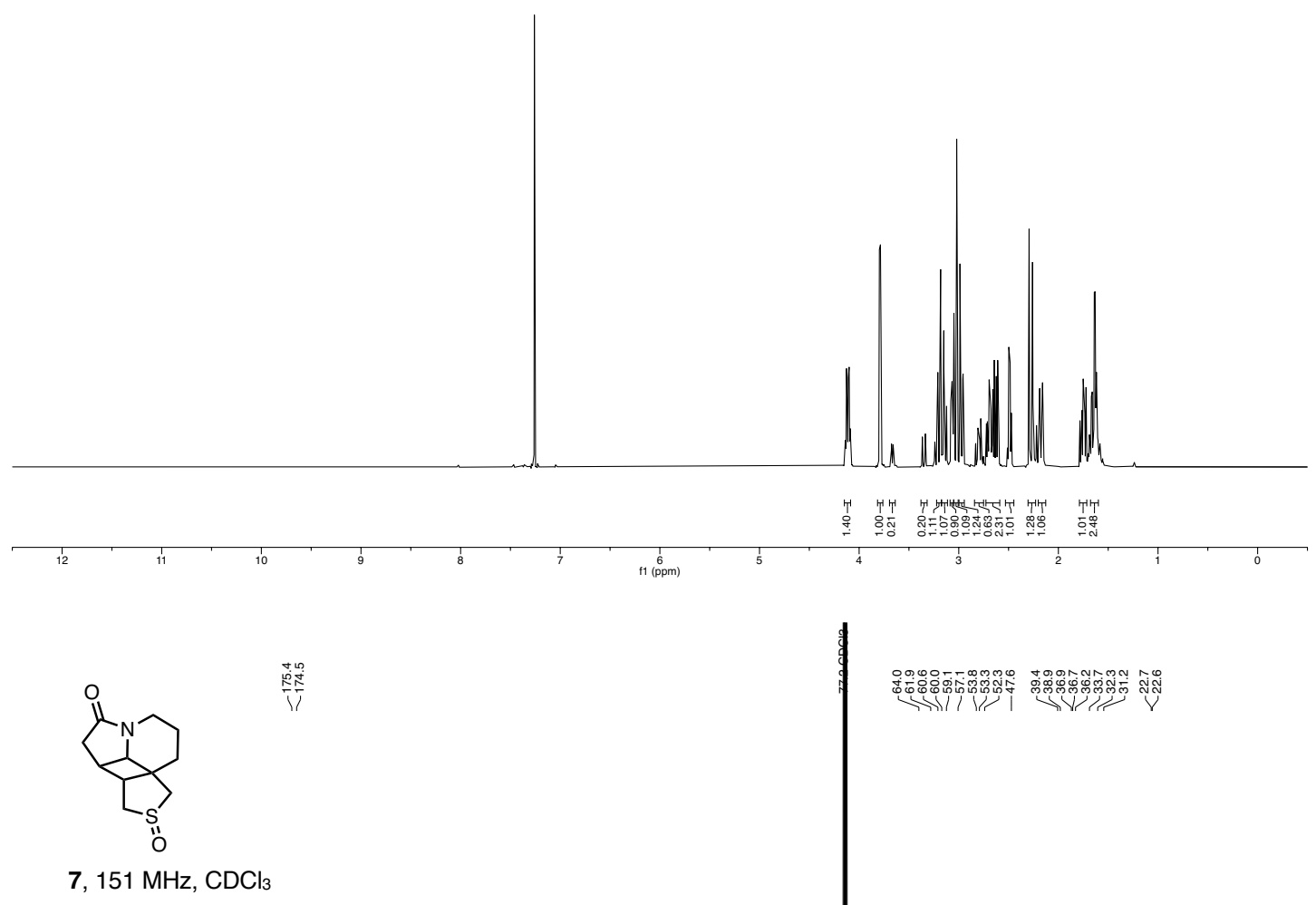

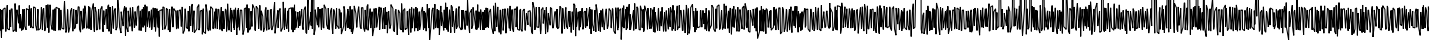

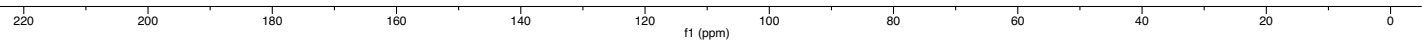




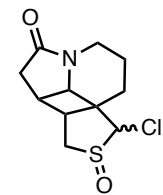

$\mathrm{S} 7,600 \mathrm{MHz}, \mathrm{CDCl}_{3}$

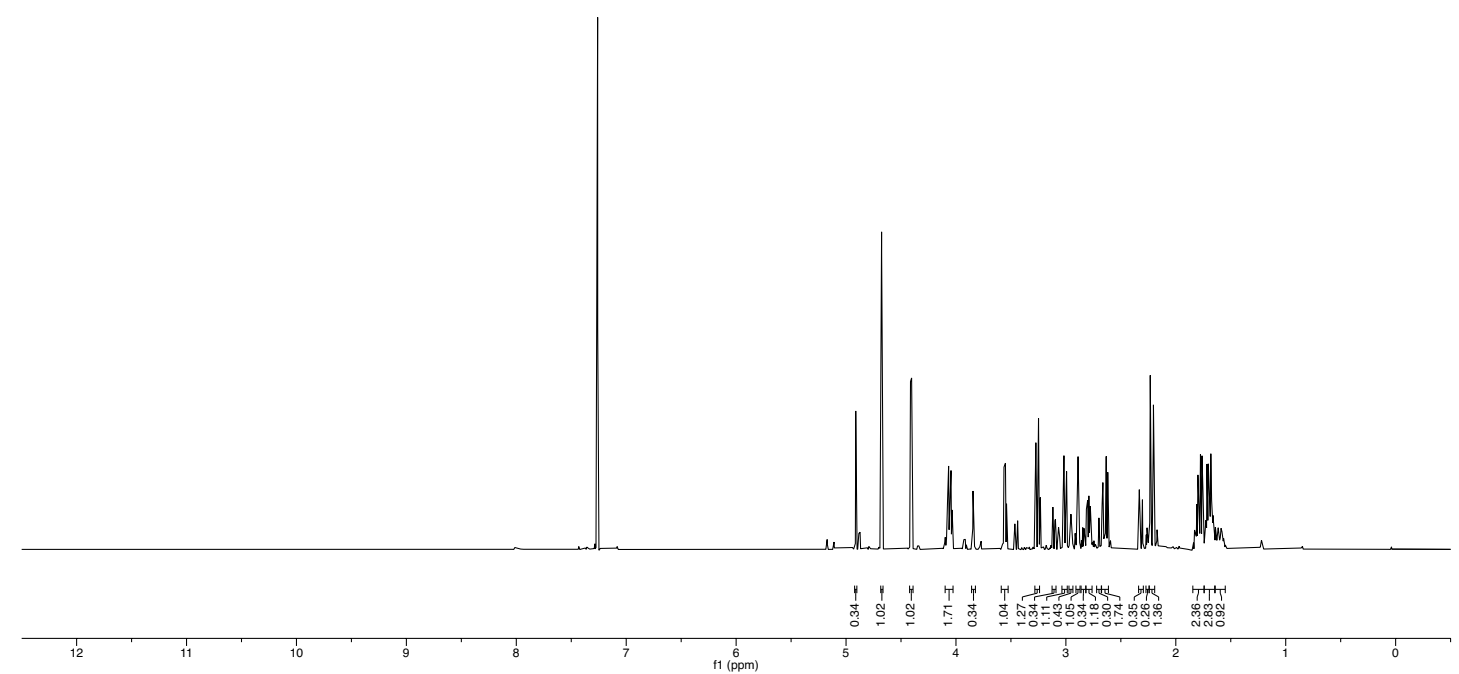

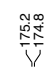

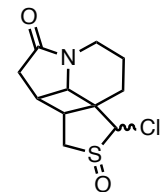

S7, $151 \mathrm{MHz}, \mathrm{CDCl}_{3}$
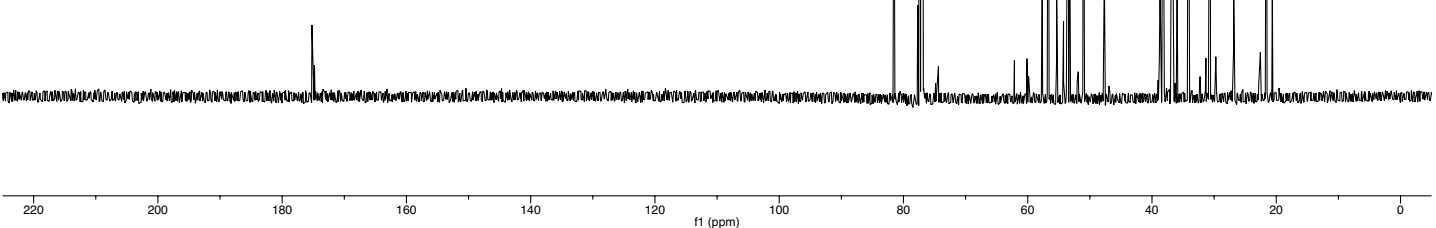


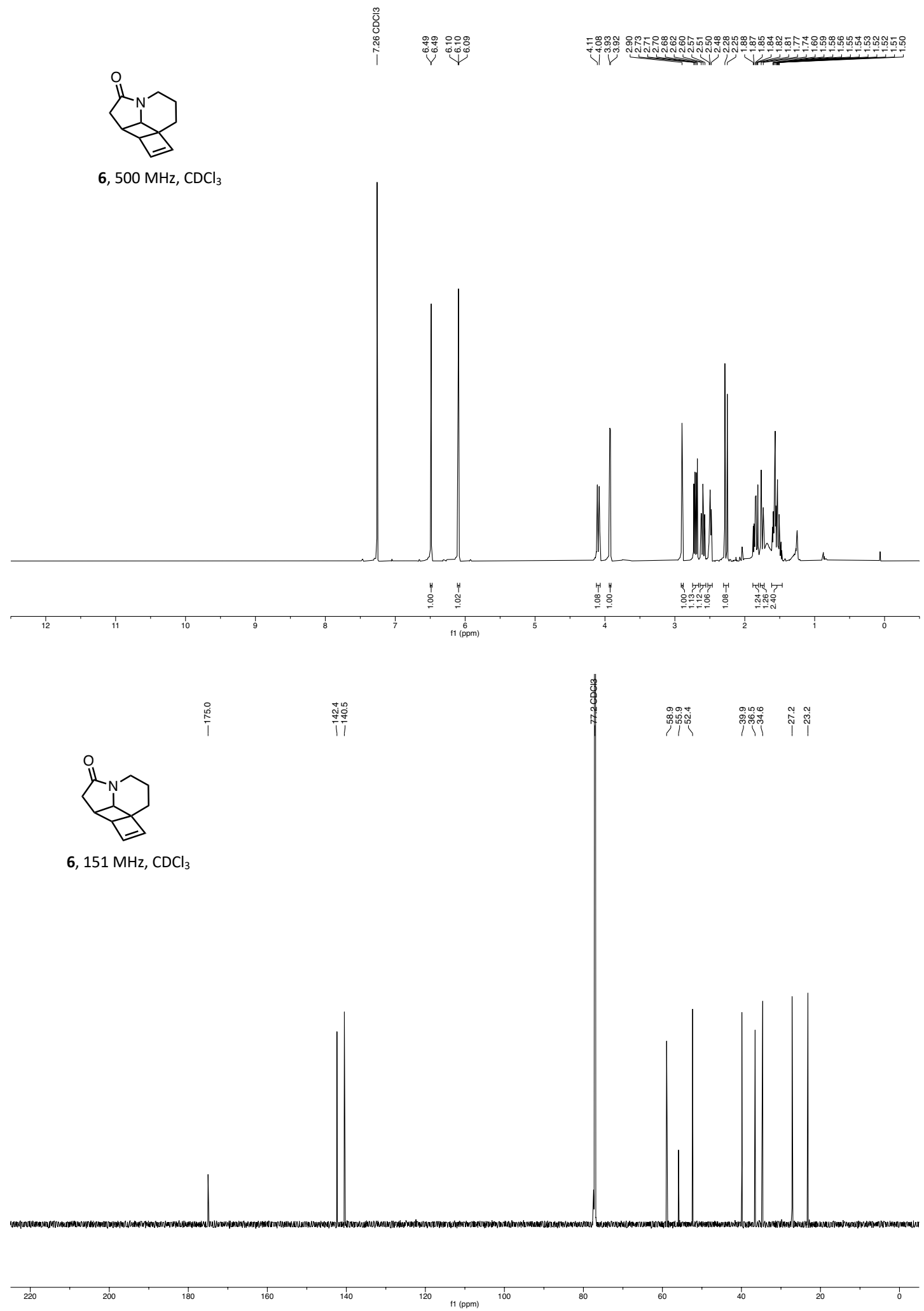




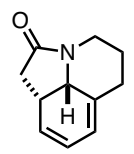

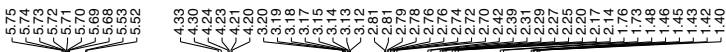

5, $500 \mathrm{MHz}, \mathrm{CDCl}_{3}$

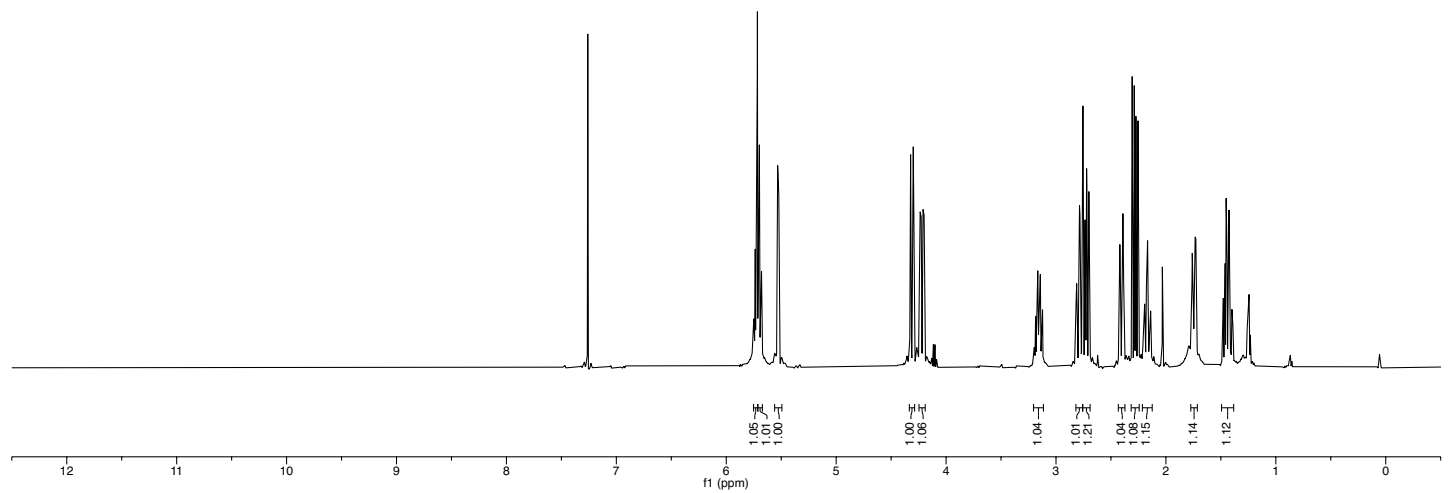<smiles>C1[Si][Ge]=[Ge]1</smiles>

5, $126 \mathrm{MHz} \mathrm{CDCl}_{3}$

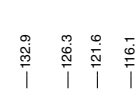

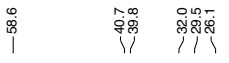

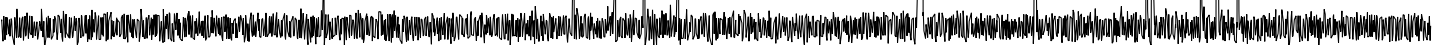

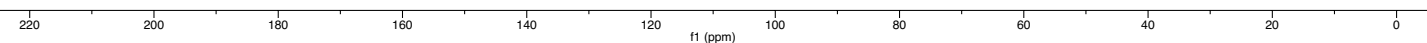




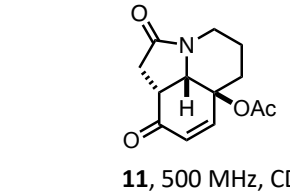

11, $500 \mathrm{MHz}, \mathrm{CDCl}_{3}$

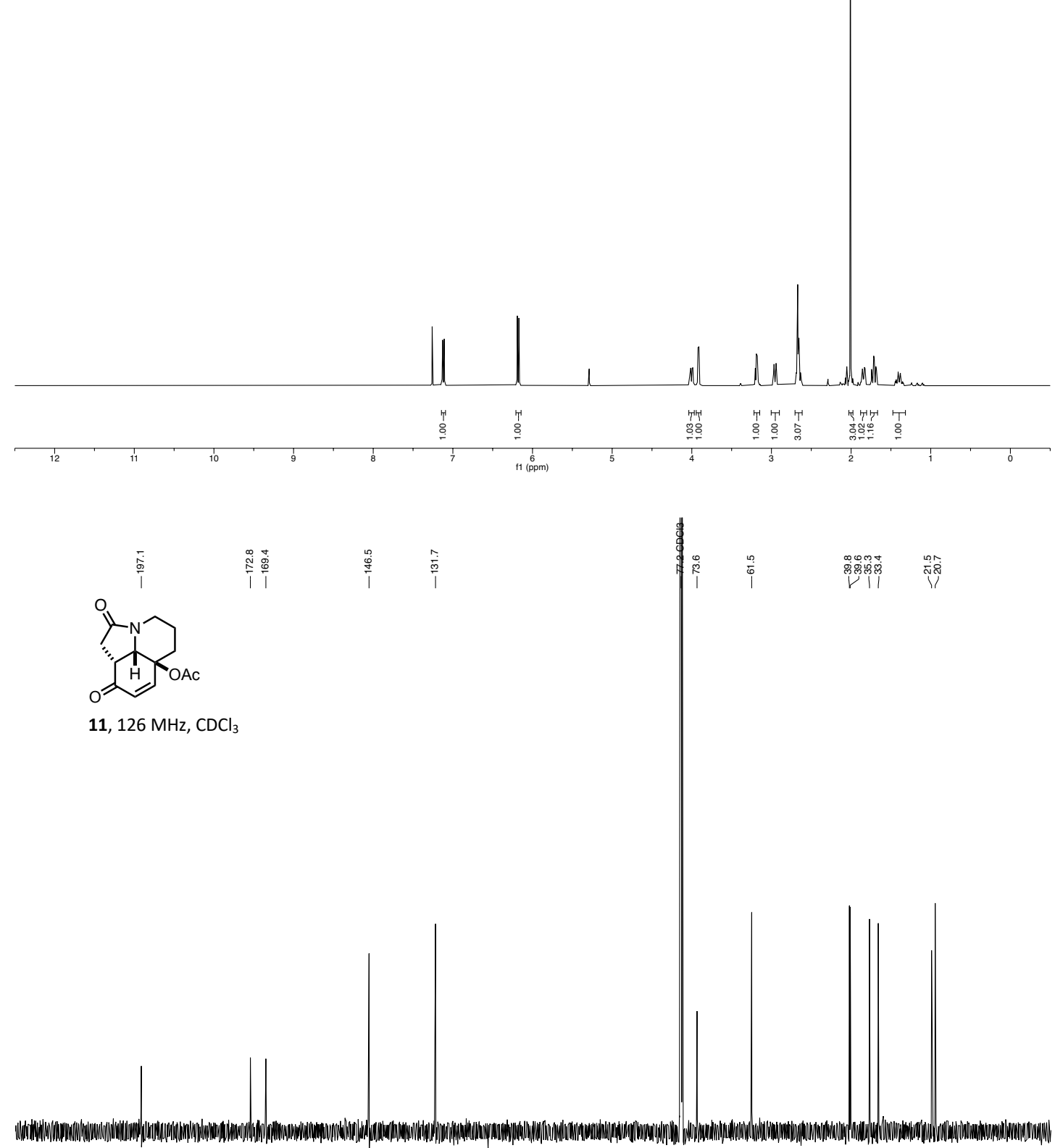

220

180

160

140

${ }_{120}^{1}+{ }_{11}^{1}(\mathrm{ppm}) \quad{ }^{1}$ 
品

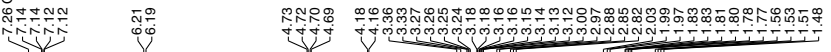

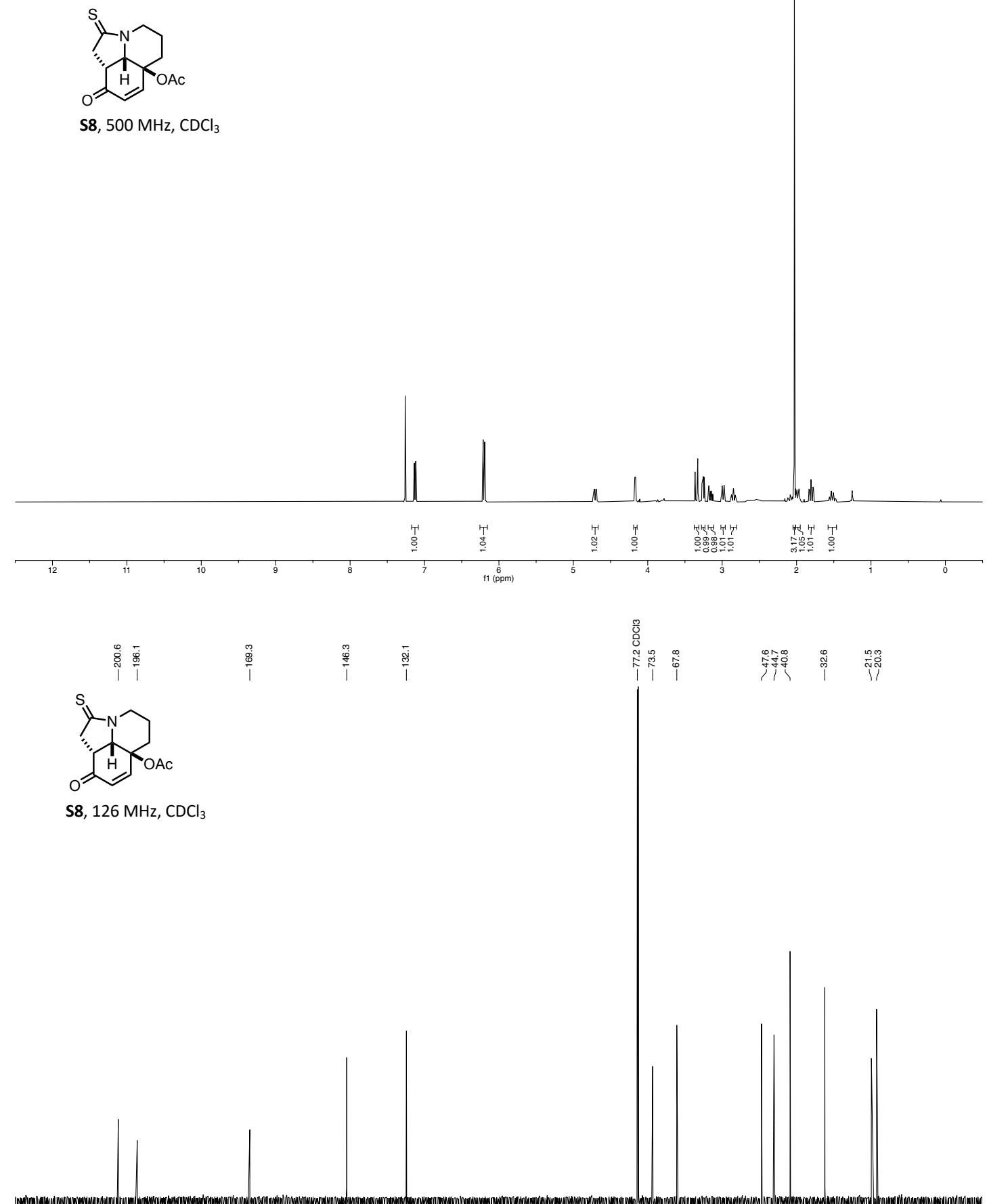

S8, $500 \mathrm{MHz}, \mathrm{CDCl}_{3}$

140

$\mathrm{f1}(\mathrm{ppm})$

${ }_{80}^{1}+\frac{1}{60}$

${ }_{40}^{10}+\frac{1}{20}$ 

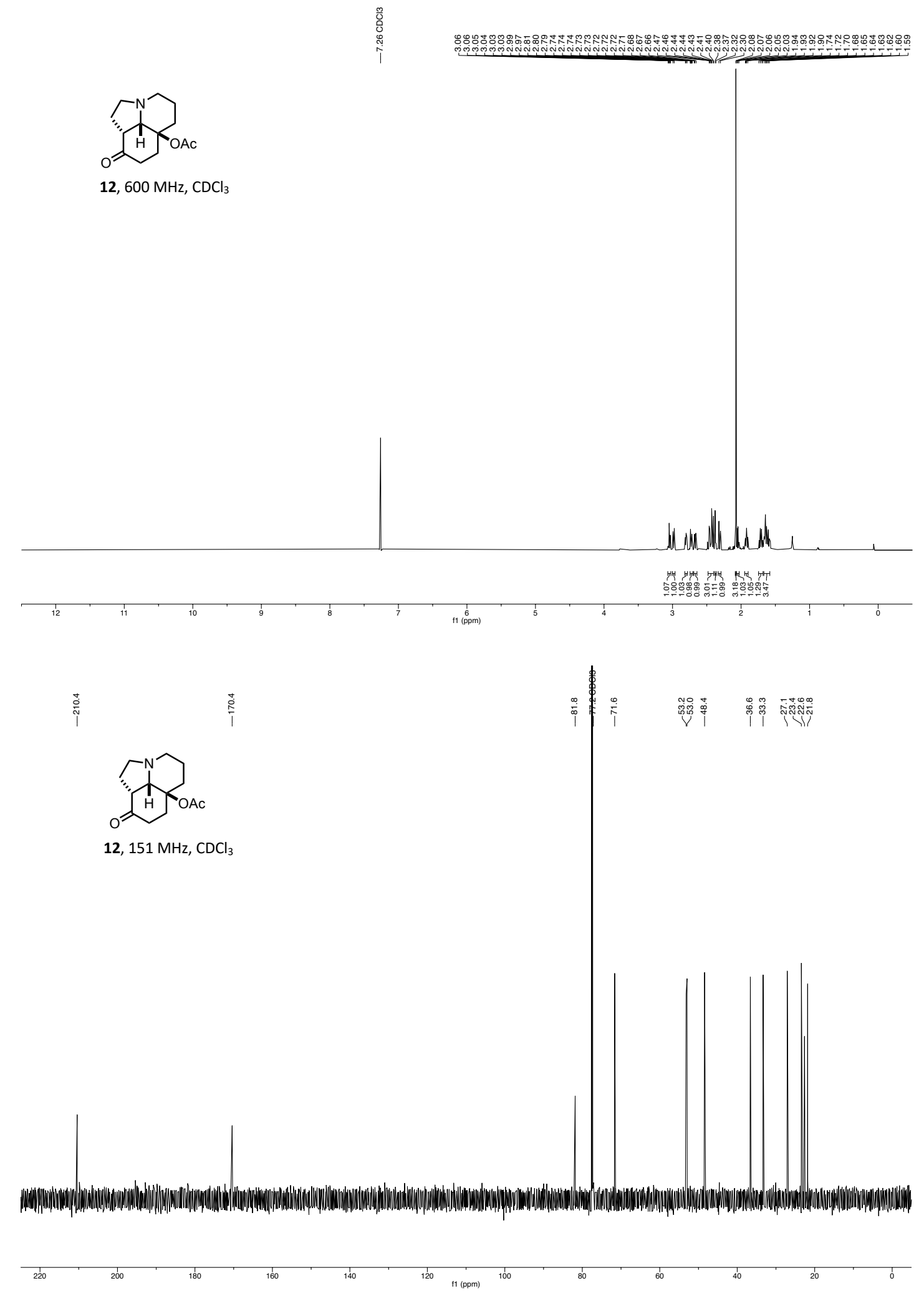


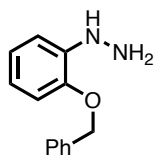

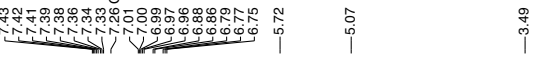

13, $500 \mathrm{MHz}^{\mathrm{CDCl}}$
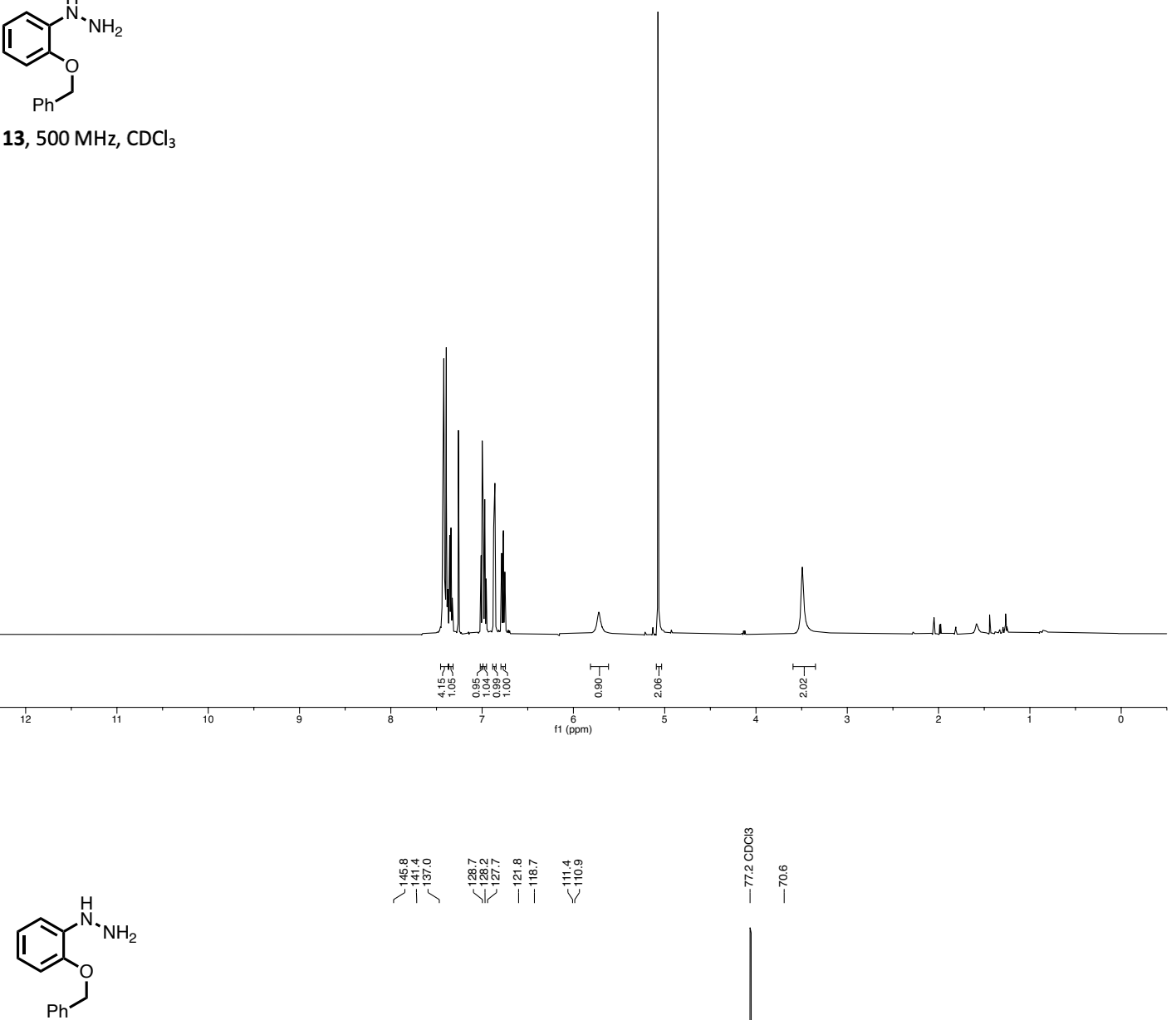

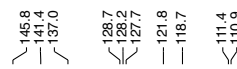

13, $151 \mathrm{MHz}, \mathrm{CDCl}_{3}$

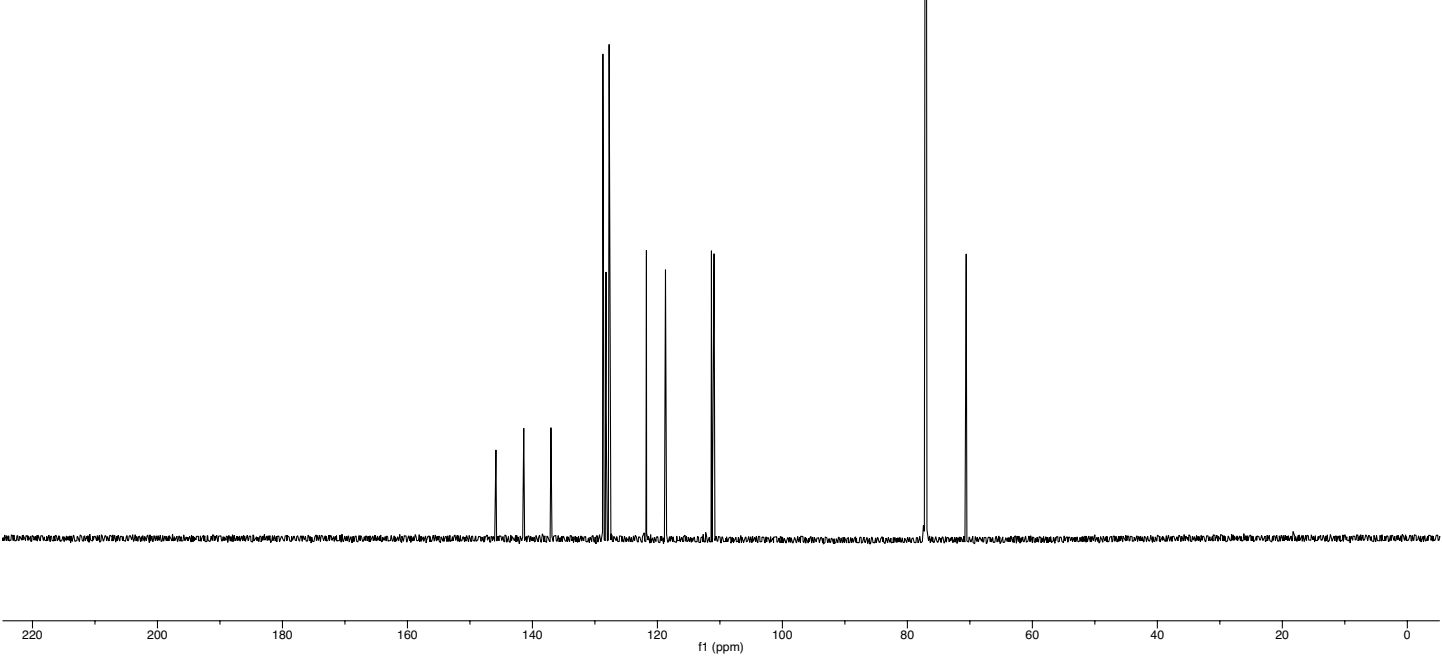



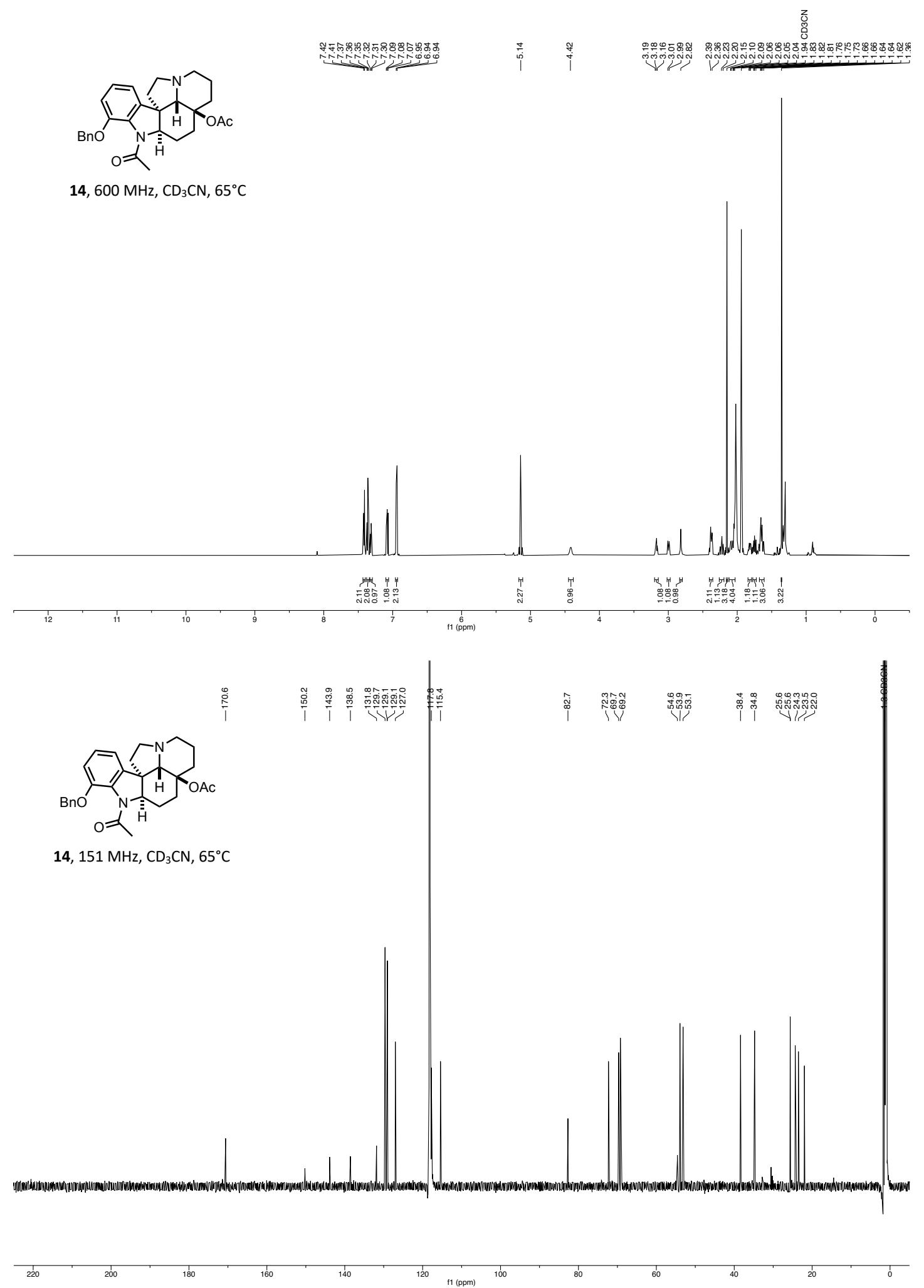

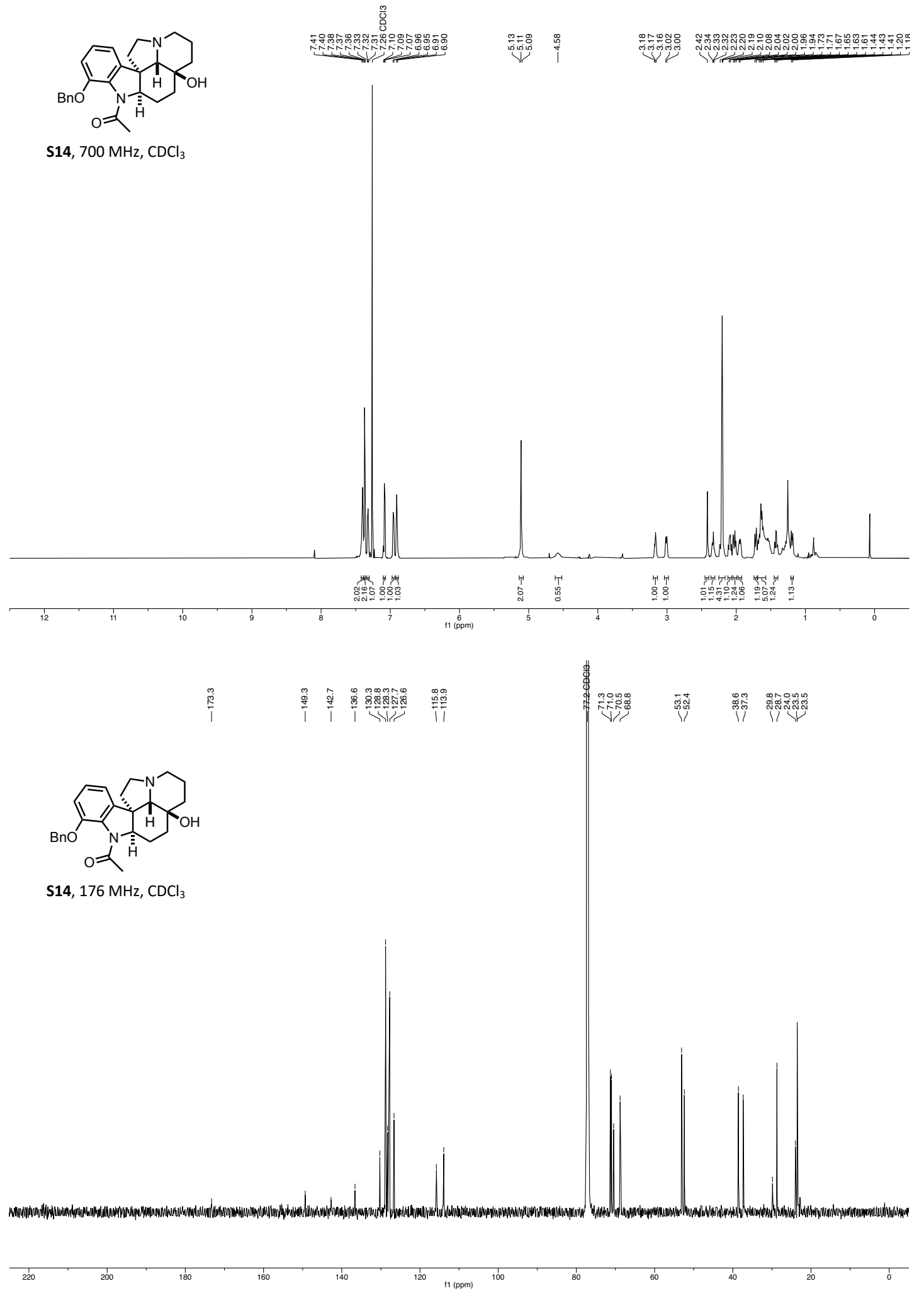
$\underset{1}{\overline{0}}$

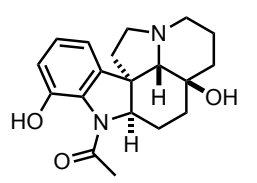

1, $700 \mathrm{MHz}, \mathrm{CDCl}_{3}$
:

กิบ

$\stackrel{8}{+}$
8ำ
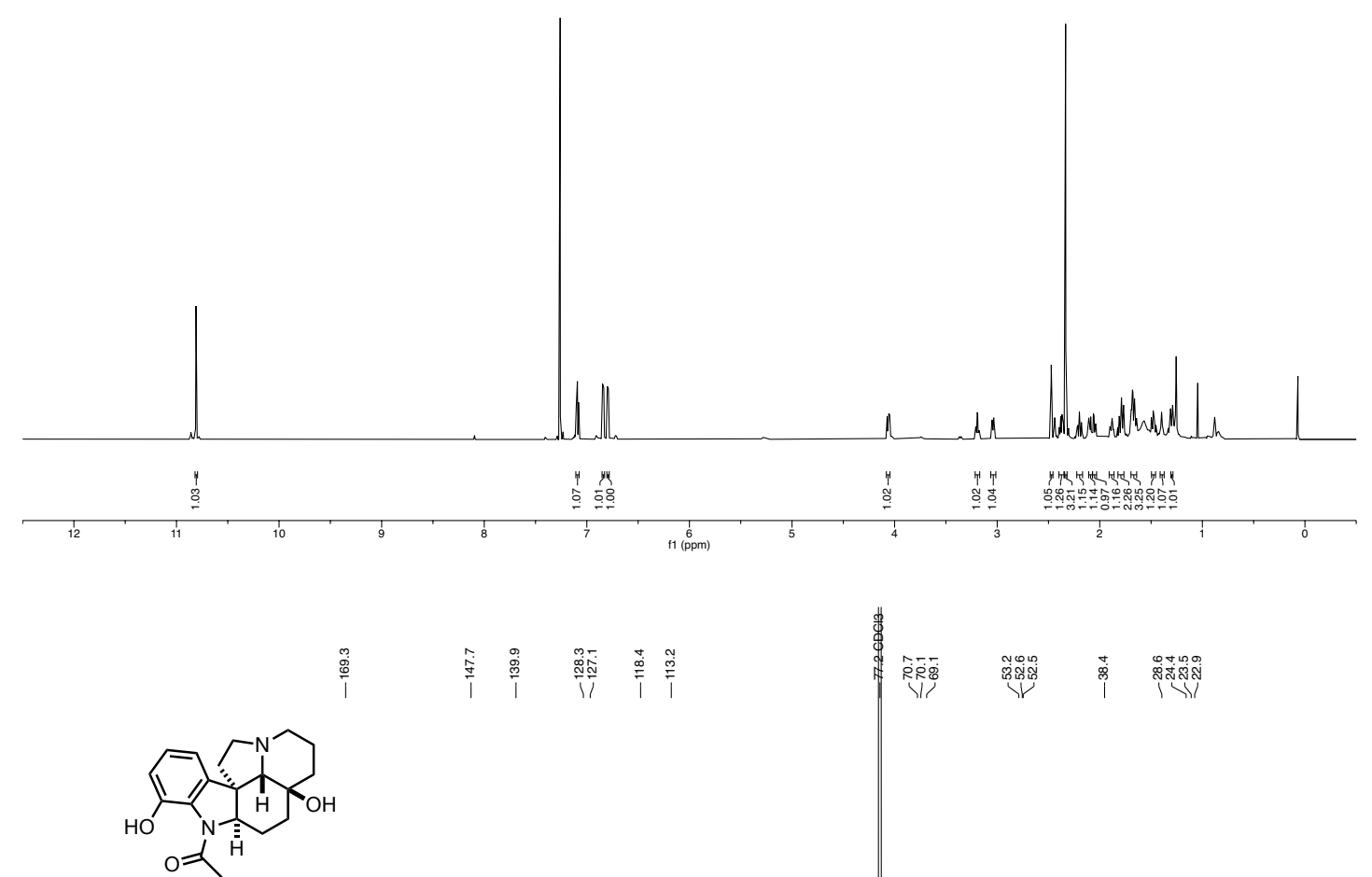

1, $176 \mathrm{MHz}, \mathrm{CDCl}_{3}$
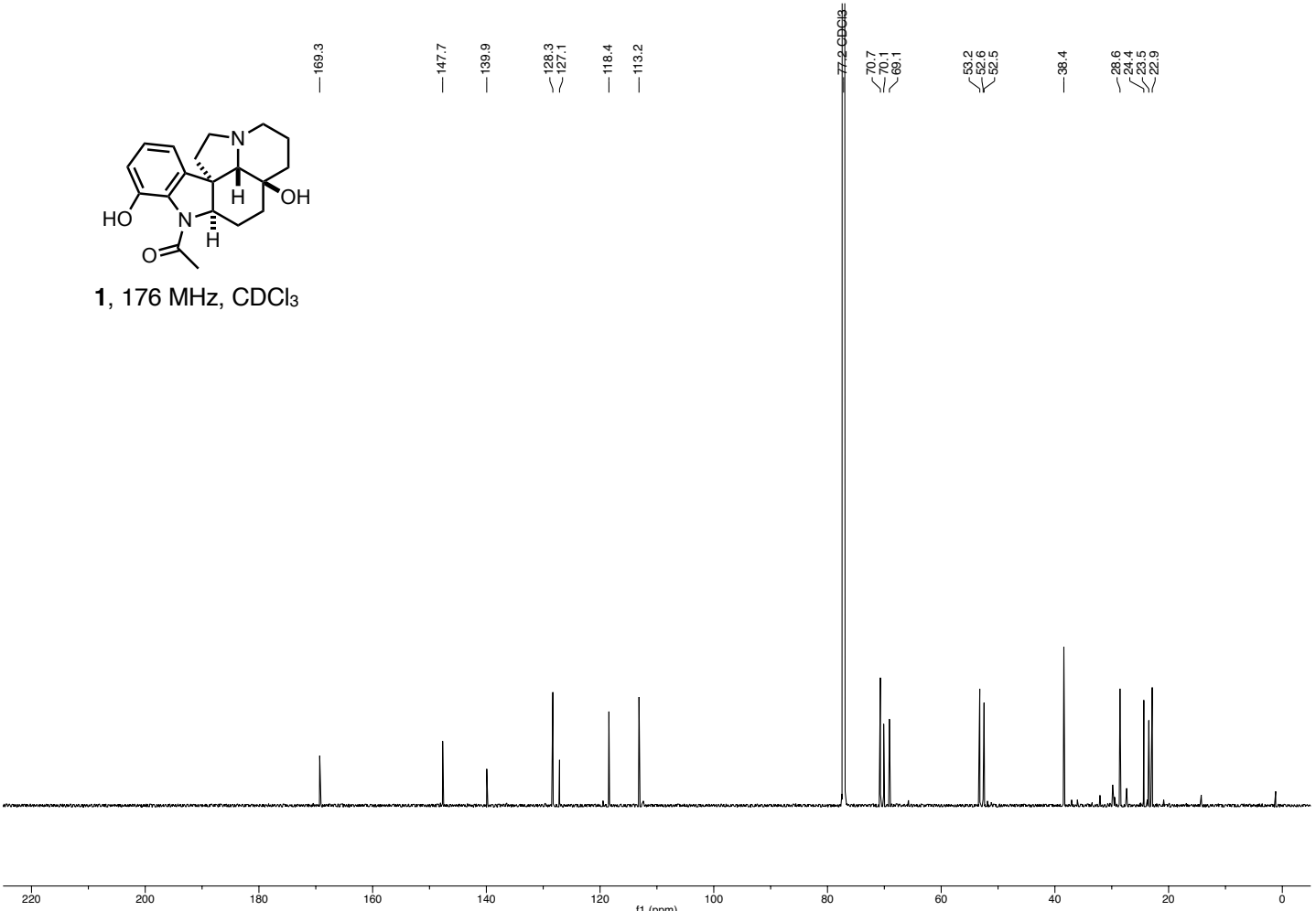
${ }^{1} \mathrm{H},{ }^{1} \mathrm{H}-\mathrm{COSY}$ of 1
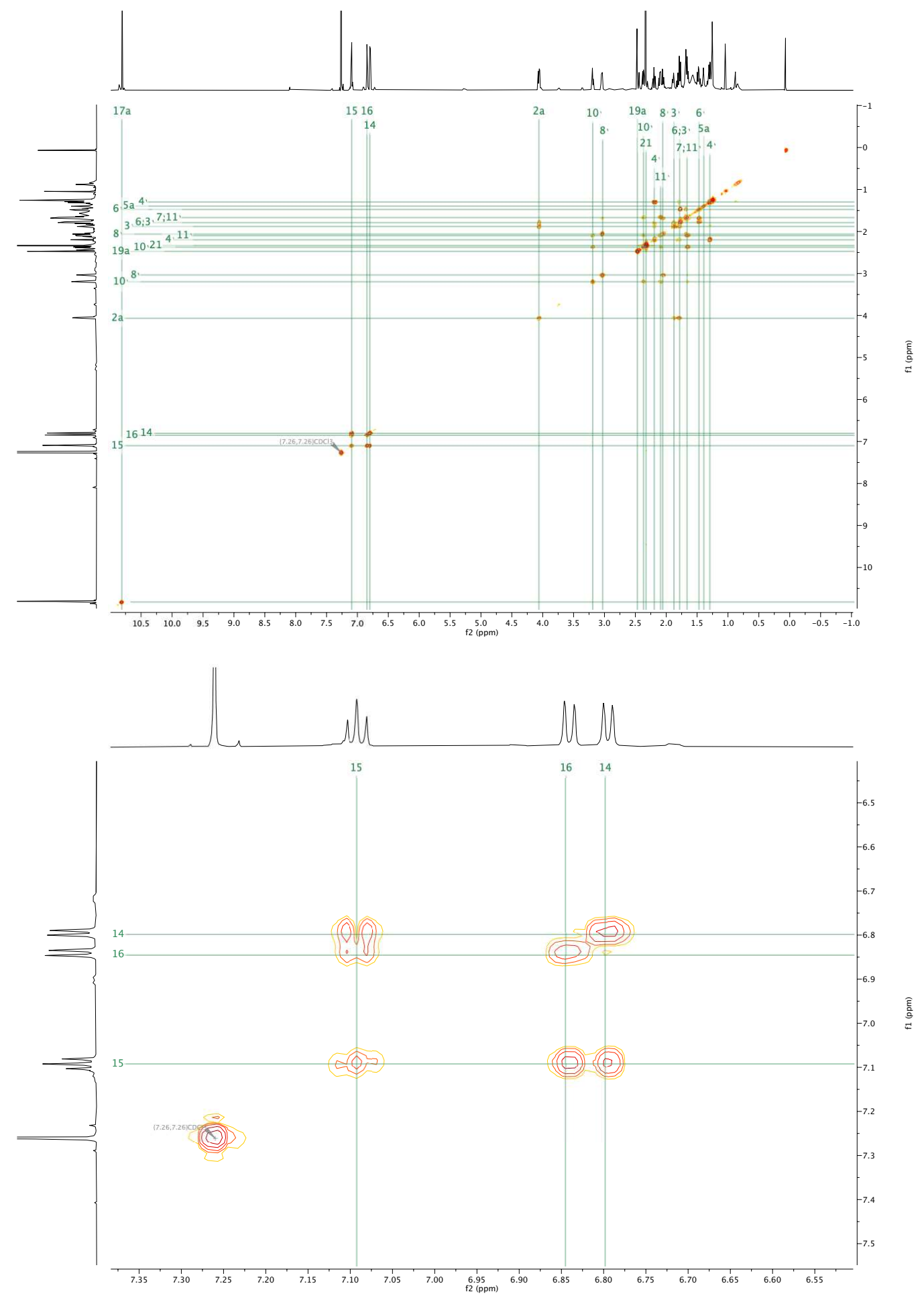

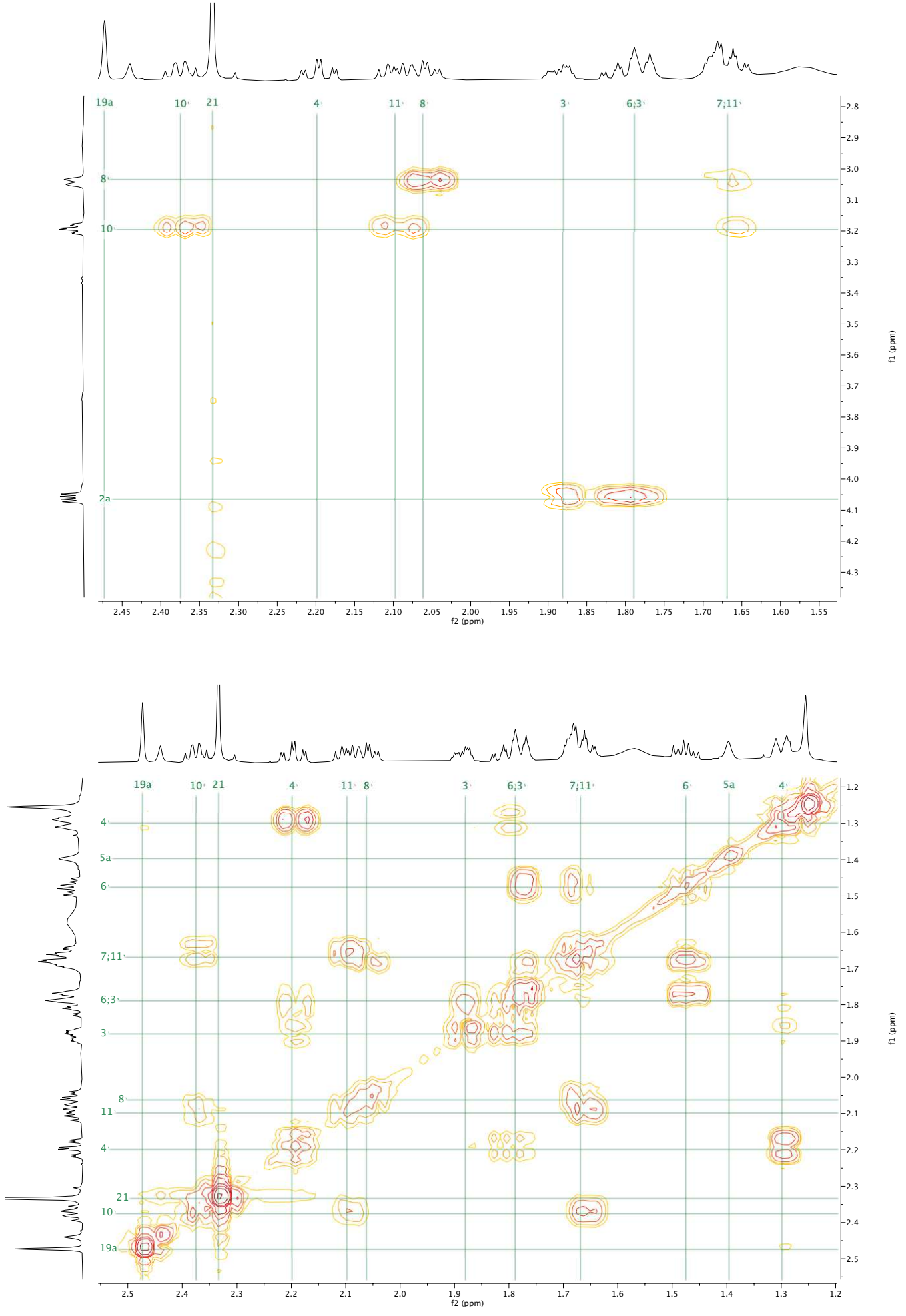
${ }^{1} \mathrm{H},{ }^{13} \mathrm{C}-\mathrm{HSQC}$ of $\mathbf{1}$
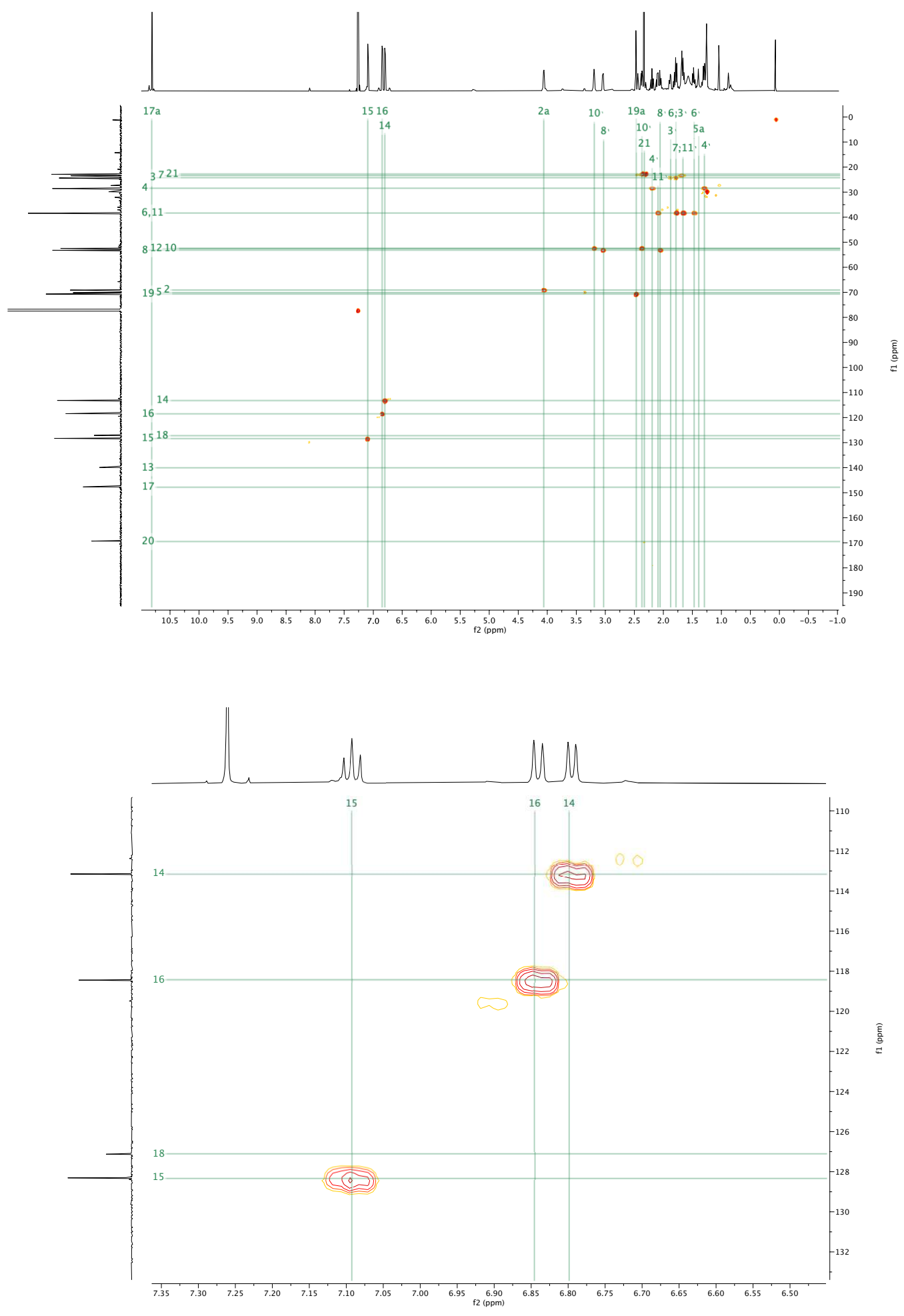

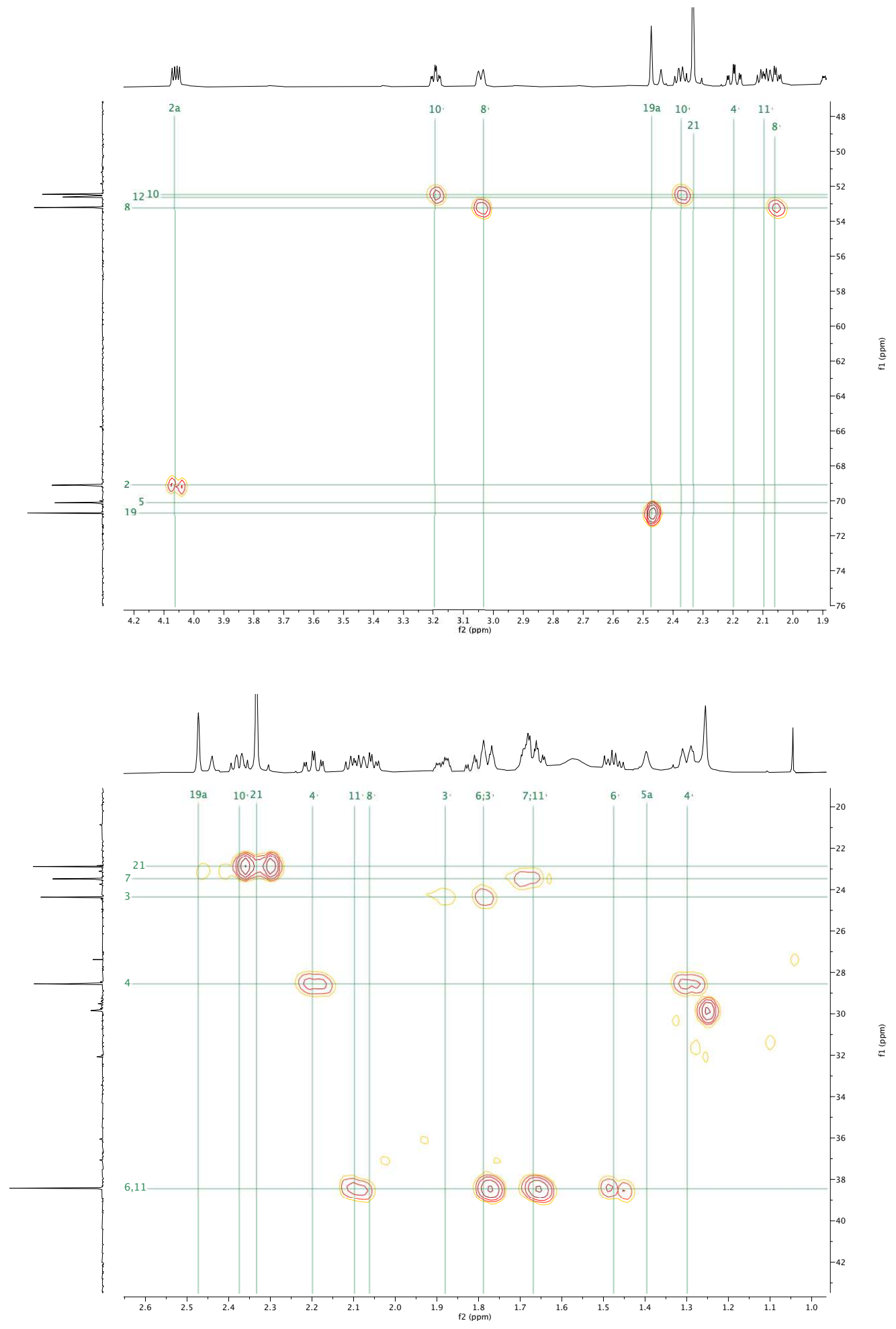
${ }^{1} \mathrm{H},{ }^{13} \mathrm{C}-\mathrm{HMBC}$ of 1
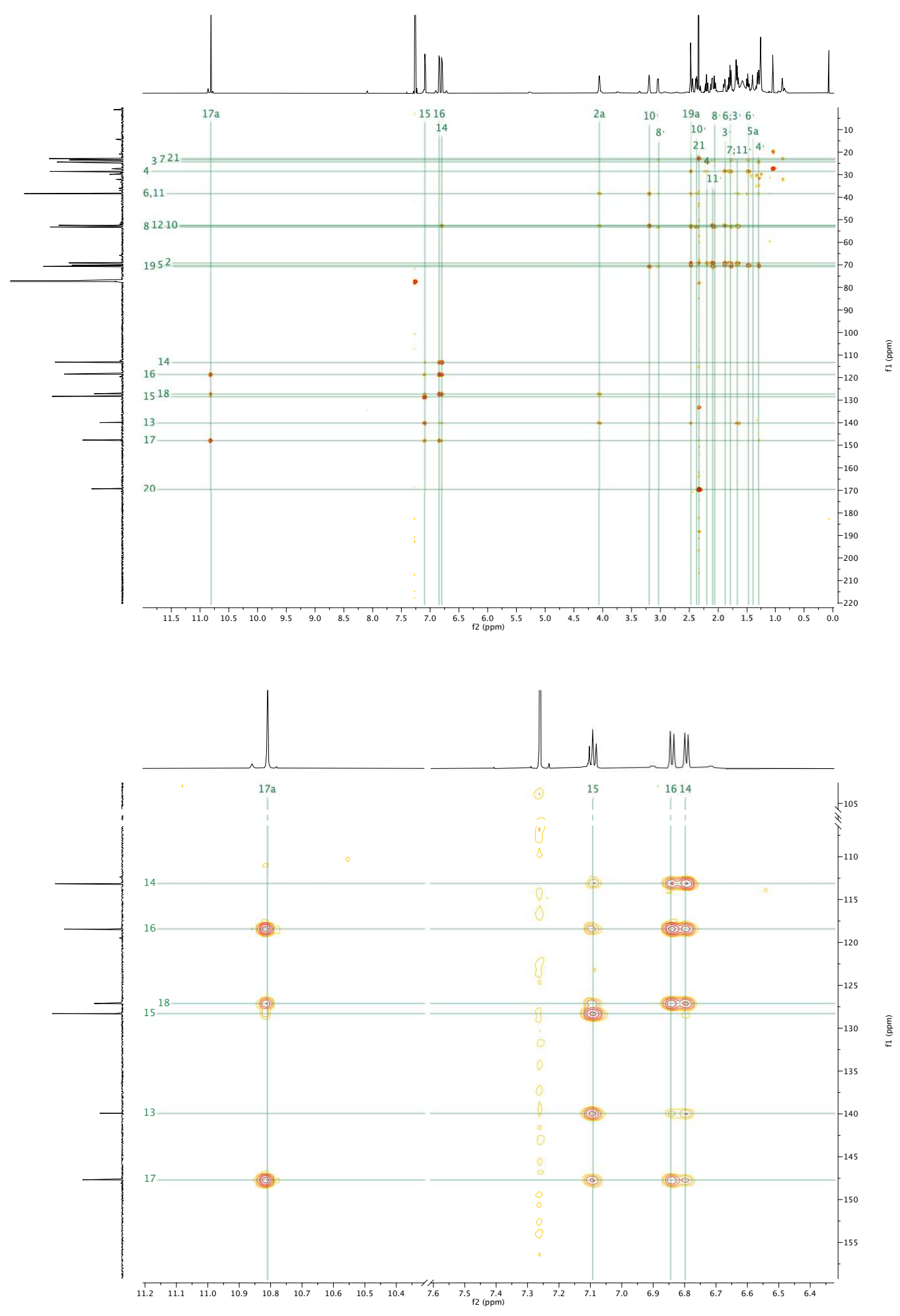

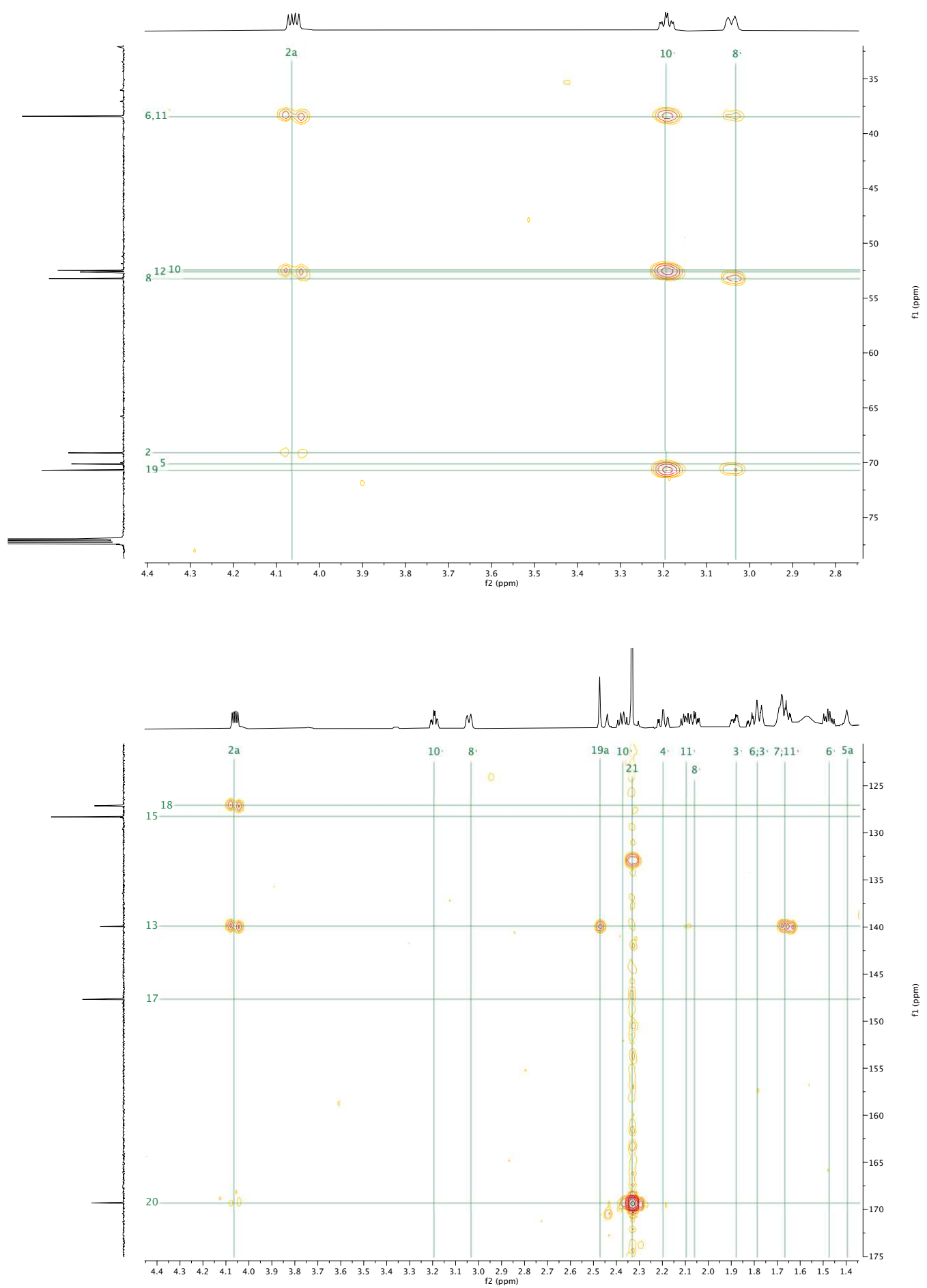

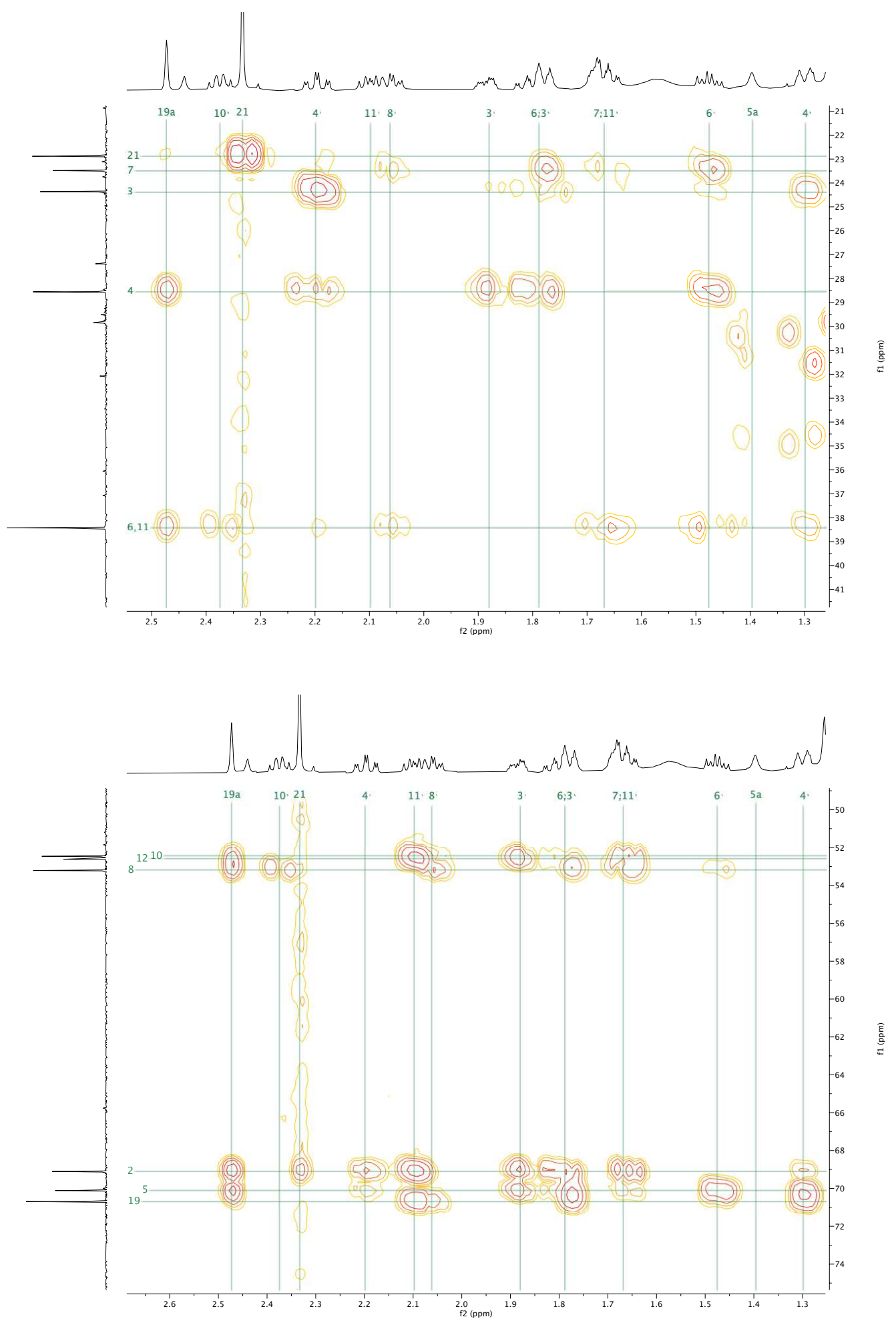
4.2 Known Compounds
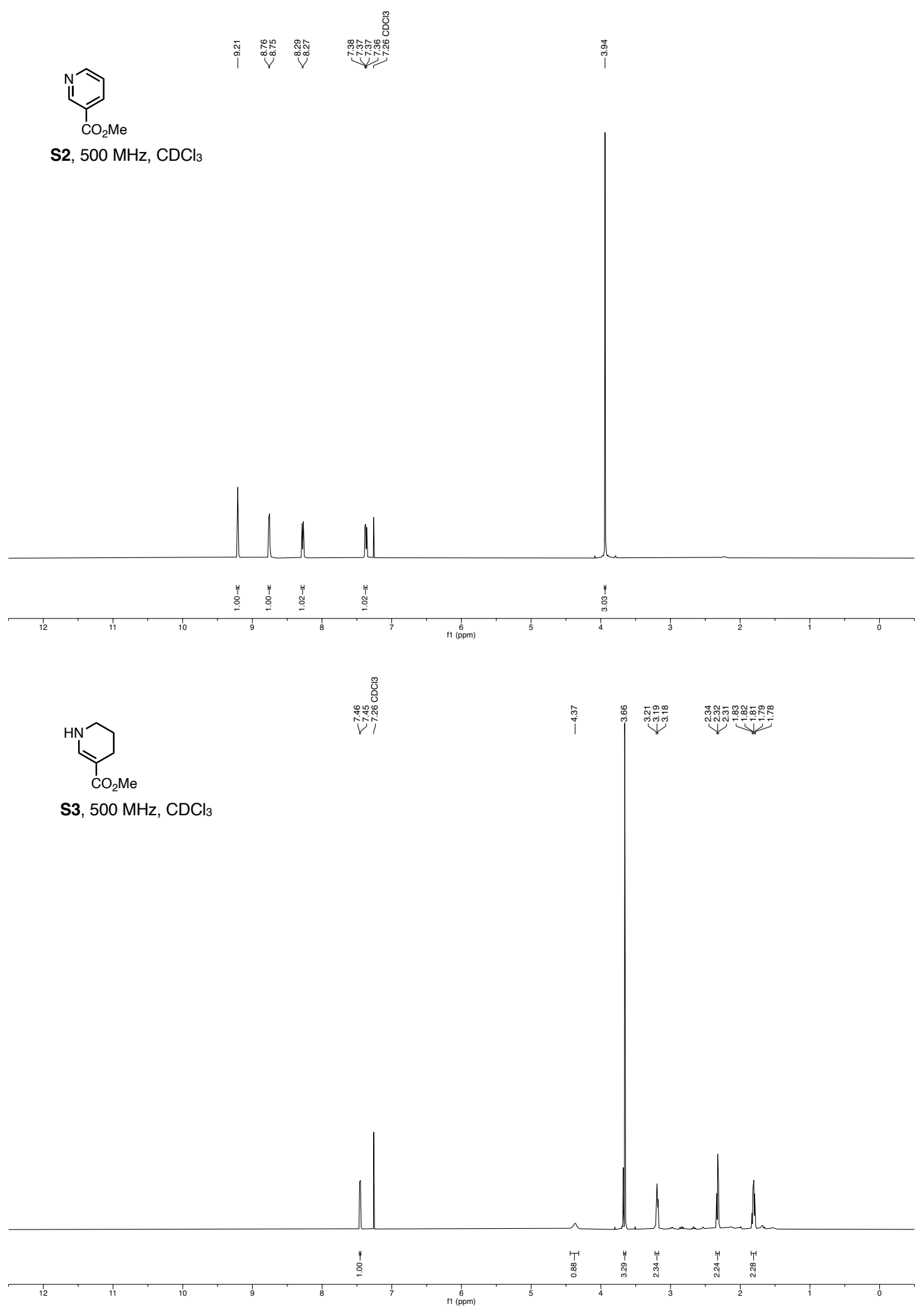

S51 


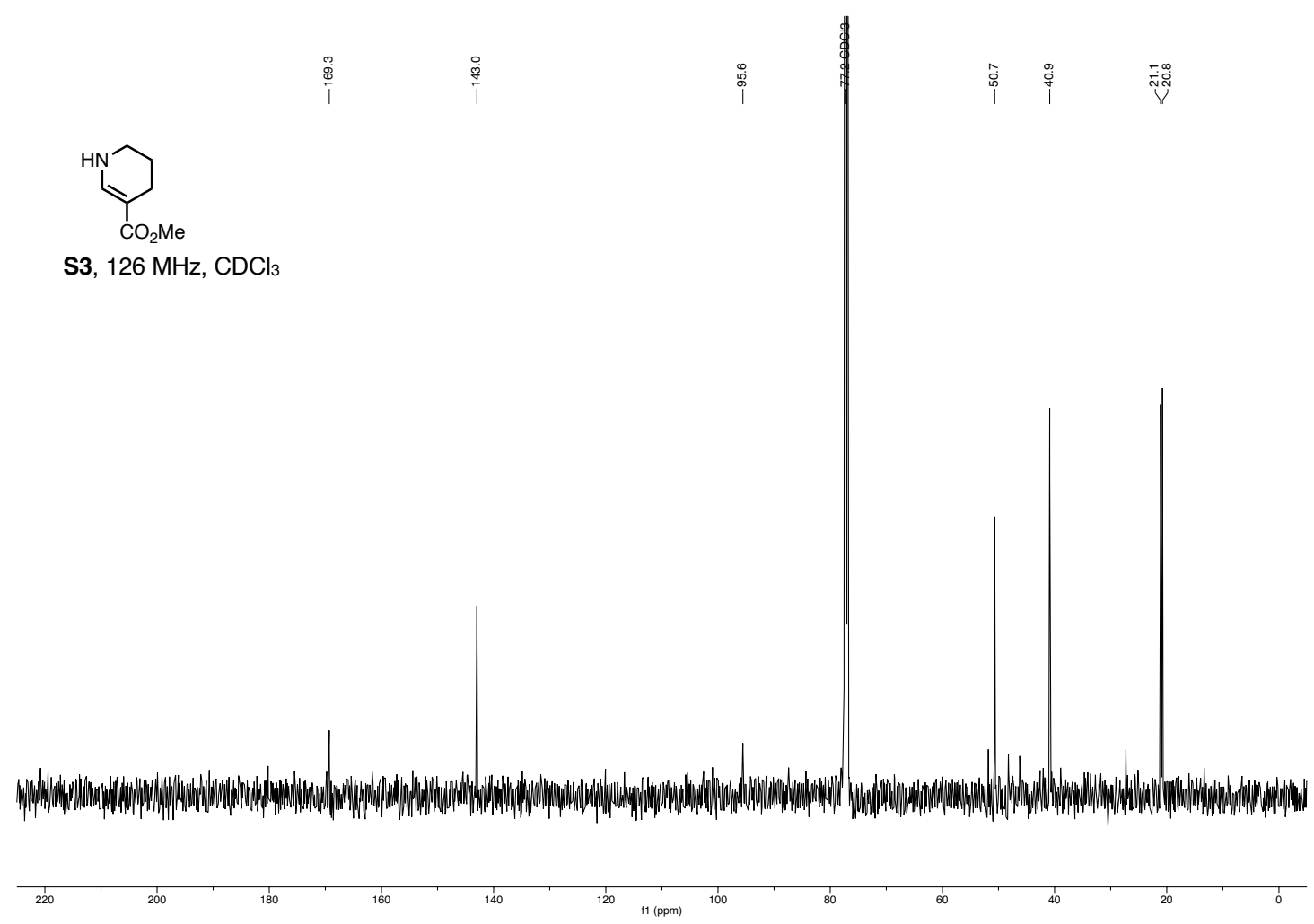

S52 
$\overbrace{\mathrm{OH}}$

$\mathrm{S} 10,500 \mathrm{MHz}, \mathrm{CDCl}_{3}$

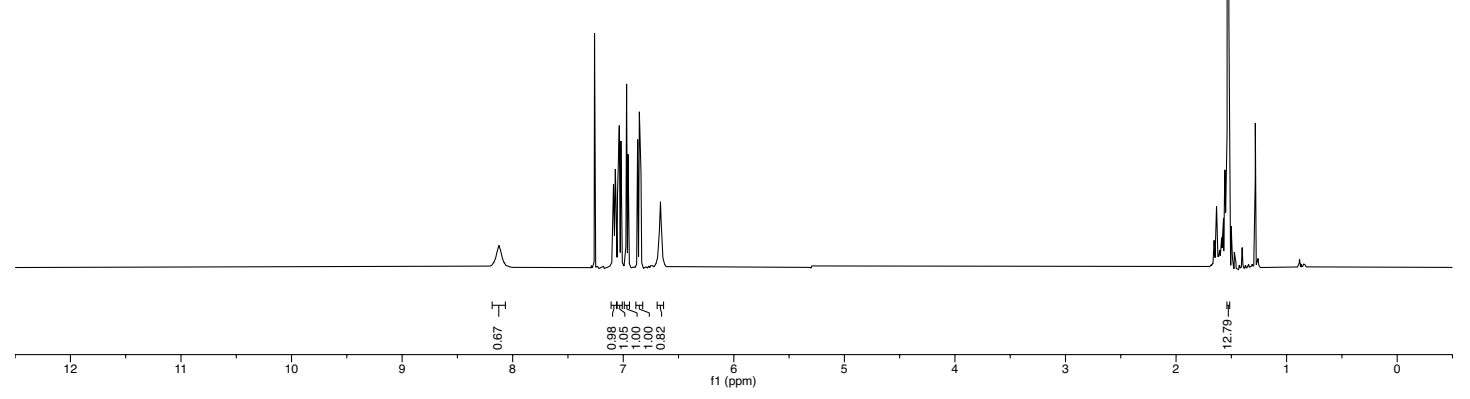

$\mathrm{NHBOC}_{\mathrm{OH}}$

$\mathrm{S} 10,126 \mathrm{MHz}, \mathrm{CDCl}_{3}$
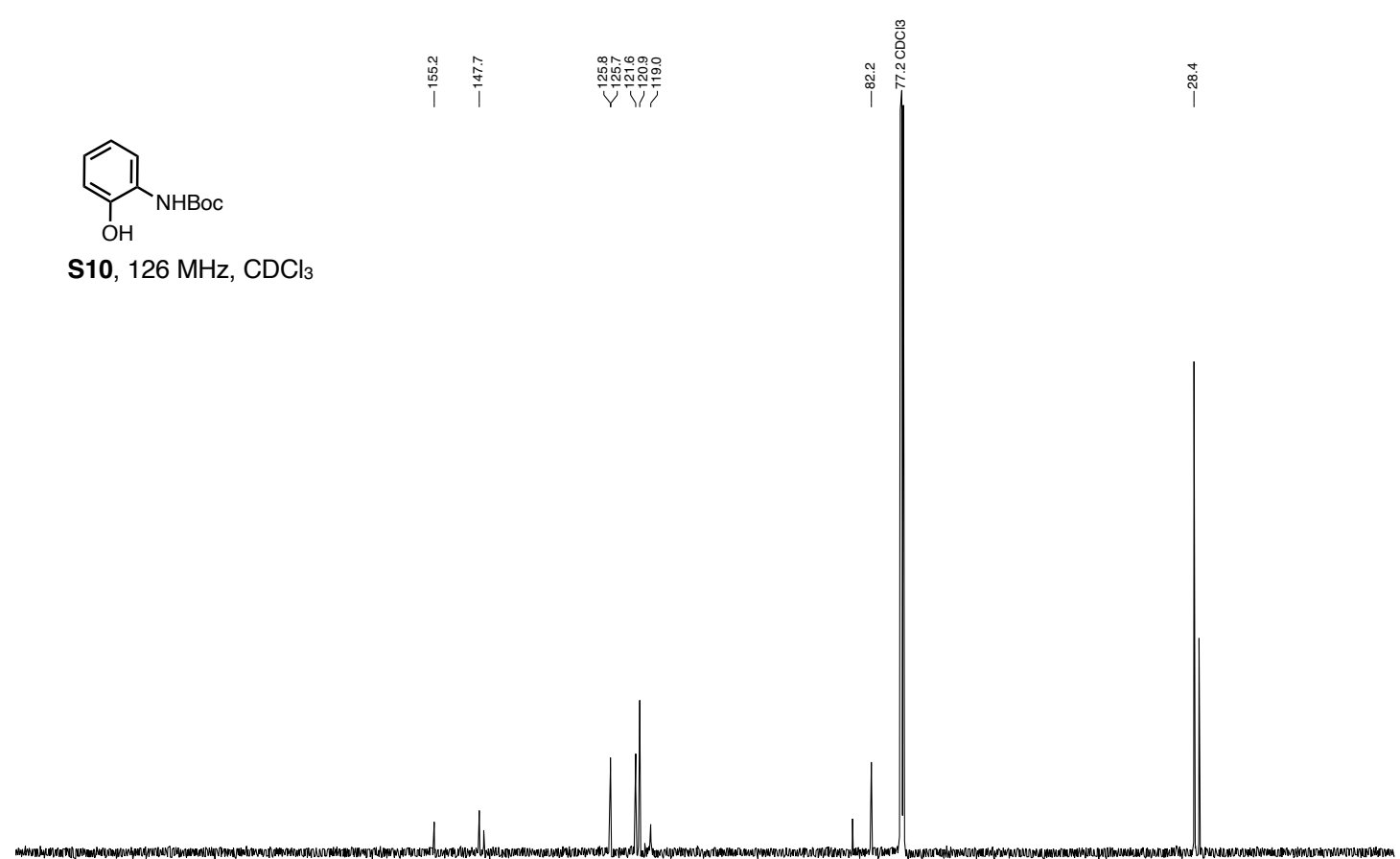

$\frac{1}{220}$

200

160

${ }_{120}^{1} \quad 100$

20 


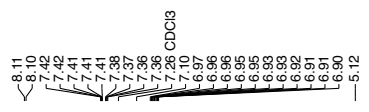

$\overbrace{\mathrm{OBn}}$

$\mathbf{S 1 1}, 500 \mathrm{MHz}, \mathrm{CDCl}_{3}$

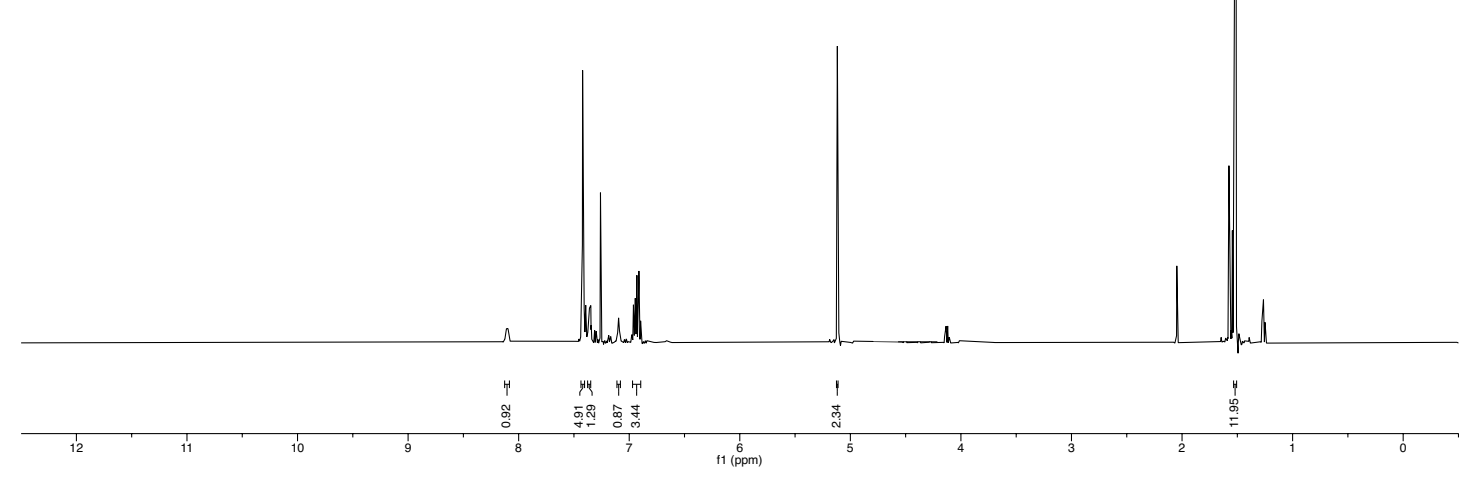

NHBoc

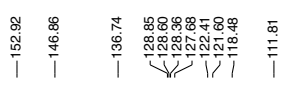

$\begin{array}{lll}0 & \\ 0 & \\ 0 & \\ 9 & \\ 9 & 0 & 8 \\ 0 & 0 & 0 \\ 0 & 1 & 0 \\ 1 & 1 & 1\end{array}$

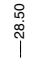

$\mathbf{S 1 1}, 151 \mathrm{MHz}, \mathrm{CDCl}_{3}$

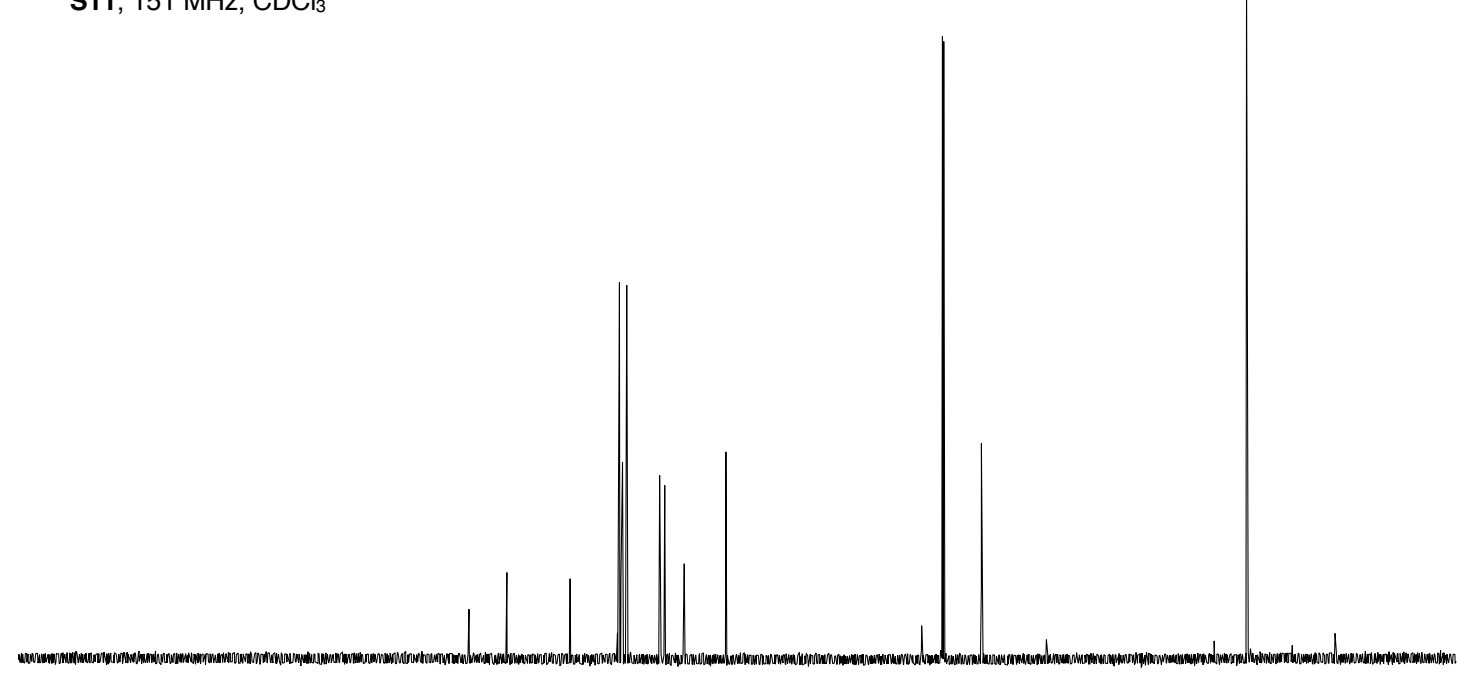

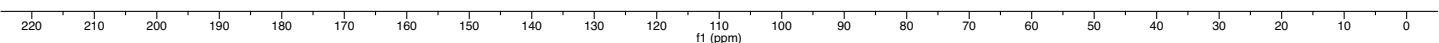




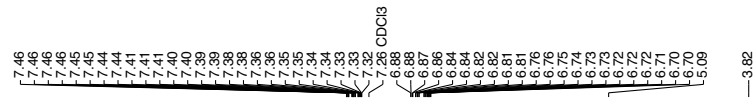

$\mathrm{OBH}_{2}$

$\mathbf{S 1 2}, 500 \mathrm{MHz}, \mathrm{CDCl}_{3}$
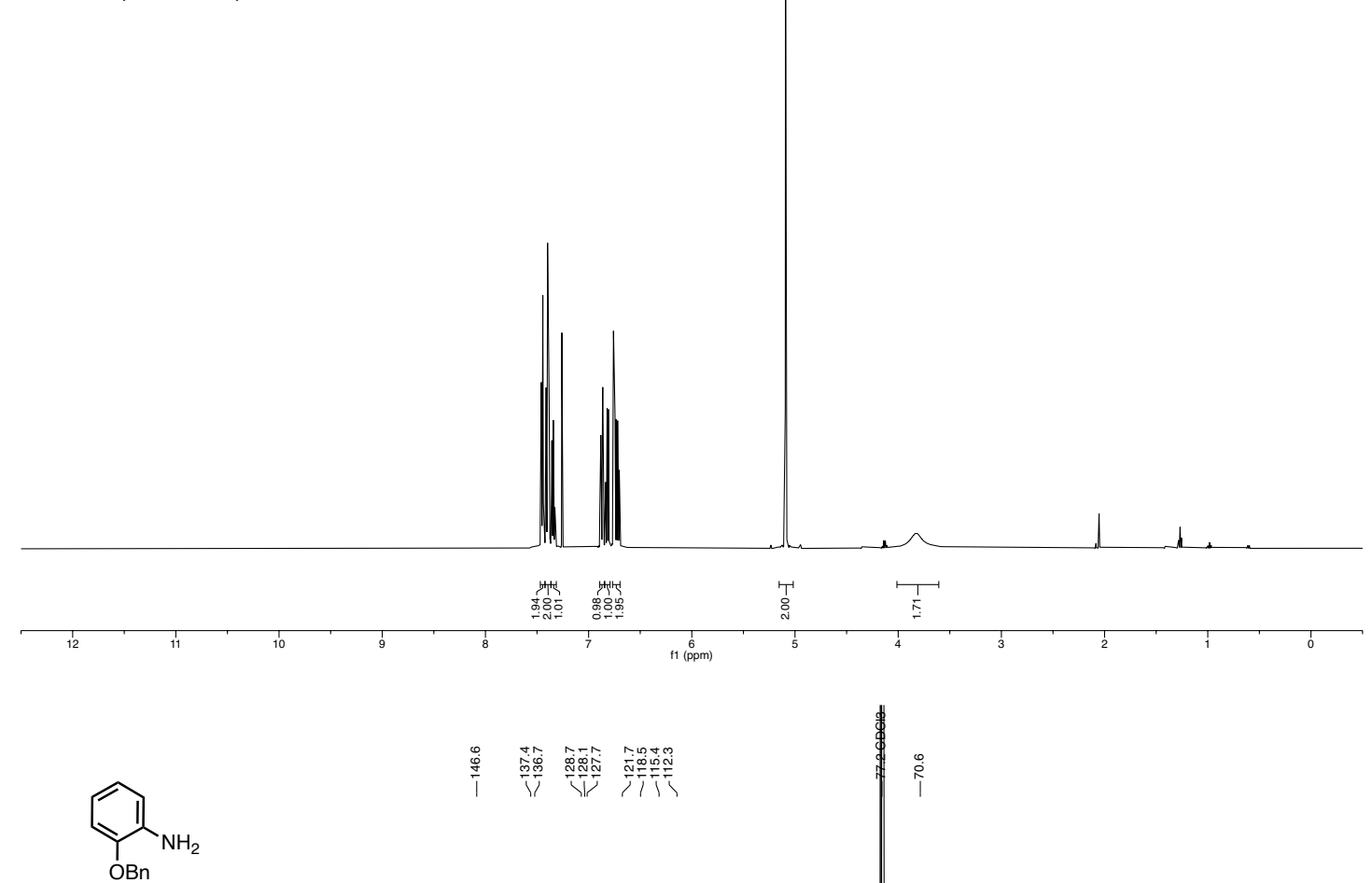

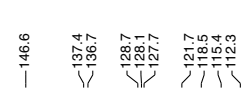

$\stackrel{\circ}{\stackrel{0}{i}}$

S12, $126 \mathrm{MHz}, \mathrm{CDCl}_{3}$

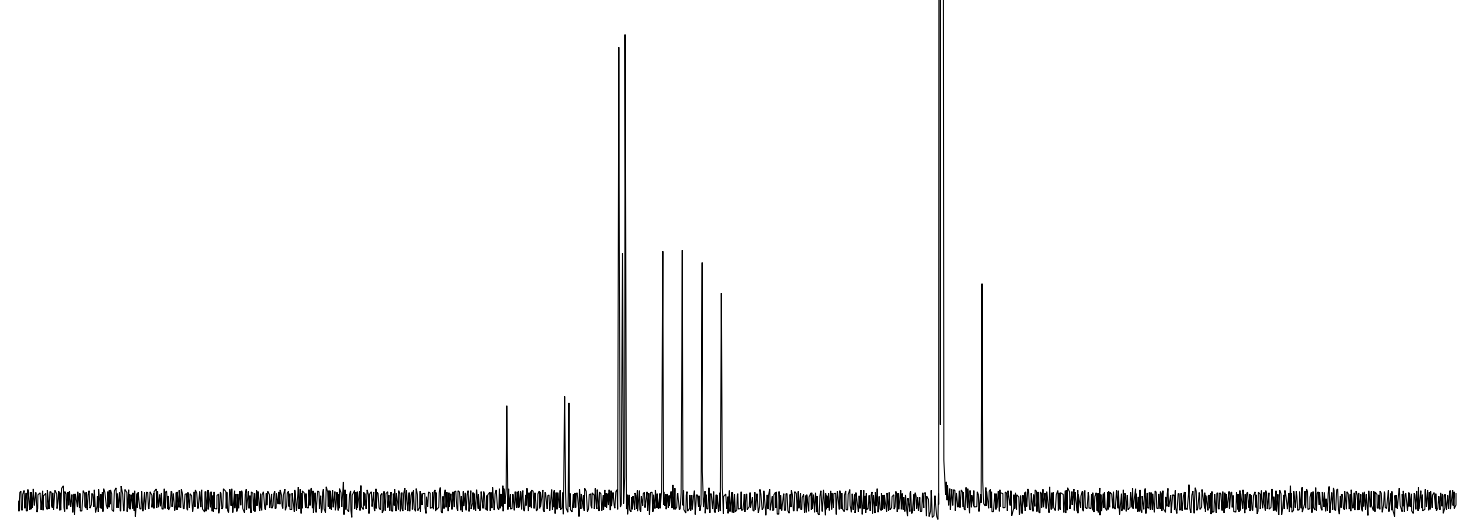

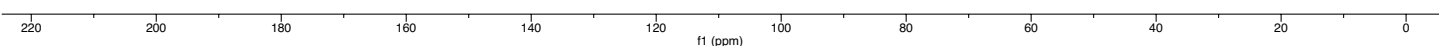

